\title{
L'usage des modules de déformation en géotechnique
}

\section{Présentation}

COMBARIEU OLIVIER Ingénieur ESTP HDR de l'université de Caen
NDLR : Les discussions sur cet article sont acceptées jusqu'au 1'r septembre 2006.
L'article d'Olivier Combarieu sur les modules en géotechnique présente l'intérèt de traiter đơn sujet diffficile mais ô combien nếcessaire dans la pratique puisqu'il est de plus en plus demandé de calculer les ouvrages géotechniques en déformation, c'est-á-dire d'en prévoir tous les principaux déplacements en donnant mieux qu'un ordre de grandeur.

Sujet difficile car chacun sait que le comportement d'un sol est complexe et fort loin de l'èlasticité linéaire gui est à la base de la résistance des matériaux. En effet, non-linéarité, non-réversibilité, dilatance, effet du temps, anisotropie sont autant cie caractéristiques ciu comportement du squelette des sols, auquel il faut adjoindre le couplage avec la pression interstitielle pour les sols fins saturés.

Sujet nécessaire car, devant cette complexité, il convient d'avoir une approche pragmatique et réaliste pour dimensionner les ouvrages géotechriques. Aprés le développement de modẻles de comportement général du sol comportant un grand nombre de paramètres et qui s'est heurté au problème de leur détermination et de leur mesure, ce sont maintenant des modéles plus simples. souvent de type élastoplastique, voire mème élastique, qui sont utilisés, mais dans lesquels le module de déformation pris en compte est adapté au type de sollicitation propre à l'ouvrage considéré. Ainsi, pour un sol donné, il n'y a pas un seul module, mais un grand nombre de modules. Reste à choisir celui qui est le plus approprie et, pour ce faire, les retours d'expérience et les résultats d'instrumentations sont d'une importance capitale.

Olivier Combarieu a passé toute sa carrière de gẻotechnicien au laboratoire régional des Ponts et Chaussées de Rouen du CEIE de Normandie Centre, Il nous livre ici son expérience, riche d’une grande diversité d'ouvracies dimensionnés et de nombreuses recherches effectuées, à propos notamment du pressiomètre dont il faut rappeler qu'il est le seul essai in situ courant qui donne un module de déformation du sol. Après avoir indiqué les différents modules que l'on peut tirer de l'essai pressiométrique. il présente une analyse critique et arqumentée de leur utilisation possible dans deux domaines oú le choix du module de déformation est capital: les tassements des fondations superficielles, puis les déplacements latéraux des parois souples et des pieux. Ses connaissances pratiques approfondies lui permettent d'apporter sur certains points un éclairage neuf, de bien montrer la complémentarité existant entre essai cedométrique et essai pressiométrique; elles l'amènent même à réfuter certaines pratiques erronées pour recommander des méthodes plus adaptées, particulièrement pour les charges de très grandes dimensions sur sols compressibles.

L'article ne comporte ni de longs calculs, ni de grandes théories. Nul doute gu'il sera apprécié de tous ceux qui, de près ou de loin, s'intéressent au calcul en déformation des ouvrages géotechniques.

François SCHLOSSER

L'auteur examine en détail la notion de module et l'usage qui en est fait dans le génie civil et tout particulièrement en géotechnique. Après un rappel élémentaire du comportement élastique et des essais initiaux en ayant permis l'étude, la réalité du comportement est décrite dans les deux domaines: celui du cisaillement (ou déviatorique), et celui de la compression (ou sphérique) dans les conditions de drainage ou non-drainage du sol. Les différents essais développés pour caractériser les massifs sont décrits, de même que les liens existant entre les divers paramètres de déformation en résultant. Trois types de problèmes très courants sont ensuite exposés, auxquels l'auteur a été confronté.

1) Tout d'abord, celui des tassements verticaux sous fondations superficielles et sous charge de très grande dímension. Est réaffirmée la pertinence, pour les sols peu compressibles, de l'approche pressiométrique pour les fondations superficielles de dimensions transversales modérées, de même que celle d'une approche élastique à partir du module cyclique issu de l'essai pressiométrique. Il est proposé un facteur correctif, non validé, dans l'approche élastique lorsque les dimensions, à la fois transversale et longitudinale, deviennent importantes. 2) Puis, celui des tassements des sols compressibles sous charges de très grande étendue où l'approche pressiométrique s'avère inadaptée et optimiste, ainsi que source possible d'erreurs graves, même si Y'on propose d'en améliorer la formulation. La méthode œdométrique reste la seule manière correcte d'appréhender le problème. En revanche, pour les autres sols où la consolidation est très faible, il est proposé de modifier, en les diminuant fortement, les coefficients a de la théorie pressiométrique afin de rendre plus réalistes les valeurs pessimistes de tassement auxquels les errements pressiométriques actuels mènent. 
Dans les deux situations, de sols très compressibles ou peu compressibles, les évaluations optimistes ou pessimistes des tassements peuvent conduire à un sousdimensionnement ou à un surdimensionnement des ouvrages géotechniques à concevoir.

3) Enfin, celui des déformées latérales des pieux ou des soutènements souples avec, pour ces derniers, des exigences très contraignantes, en zone urbaine par exemple. L'appréciation des mouvements, souvent imposés, nécessite une amélioration permanente des modèles de comportement du sol, accompagnée d'observations indispensables au calage de ceux-ci et au choix des valeurs pertinentes des umodules de déformation ») à y entrer.

Le cas des tunnels, soutènements particuliers, n'est pas examiné ici. Le rôle du module y est certes important, mais vis-à-vis des déformations à venir, l'état initial des contraintes naturelles au repos dans le massif avant creusement, par le biais du coefficient $\mathrm{K}_{\mathrm{p}}$, peut s'avérer plus important encore. Le choix de la valeur de $\mathrm{K}_{0}$ comme celle du module est donc indispensable.

Enfin, l'auteur s'est beaucoup attaché à rappeler les domaines d'utilisation respectifs de deux essais fondamentaux utilisés en France, l'œdomètre et l'essai pressiométrique.

Mots-clés: module, élasticité, sol, tassement, fondation superficielle, essai triaxial, œedomètre, essai de plaque, pressiomètre, essai dynamique.

\section{Use of deformation moduli in geotechnical practice}

The author describes the different existing tests and methods to determine the deformation modulf of a material. At first, the elastic linear isotropic material is examinated. Then, the description of soil, of his behaviour and the great difference with the elastic solid, bring to relate in detail the diversified types and natures of tests which give varying deformation characteristics whose complex relations are explained. The everyday geotechnical deformation problems are afterwards reviewed :

- the calculation of shallow settlement with pressuremeter theory and elasticity theory. It is showed how conciliate the two approaches with using the pressuremeter test;

- the settlement calculation, under wide extent loads, of soft compressible soils for which the only oedometric method is valid; the pressuremeter method, as showed with examples, is inadequate;

- finally, the lateral deformations of diaphragm walls, for which the choice of moduli from pressuremeter tests, in finite elements methods, require to much increase the $\frac{E M}{\alpha}$ values, as yet esteemed.

Key words: modulus, elasticity, soil, settlement, shallow foundation, triaxial test, oedometer, plate bearing test, pressuremeter, dynamic test.

\section{Introduction}

La notion de module est directement liée à celles, conjointes, de déformation et de contrainte appliquées à un corps. Un corps donné possède un module d'autant plus élevê que la déformation résultant de l'effet d'une force imposée (donc de contraintes imposées) est plus faible; mais la complexité que recouvre le terme de module est grande, puisque la plupart des

matériaux, surtout en géotechnique, cumulent le plus souvent des propriétés élastiques, plastiques et visqueuses, pouvant se manifester conjointement, auxquelles s'ajoutent des effets dus au fluage et à la fatigue.

En géotechnique, les règlements européens récents le prescrivant, on s'oriente progressivement vers une justification des ouvrages en déformation et déplacement. On se contentait le plus souvent, jusqu'à récemment, de justification à la rupture, l'application de coefficients de sécurité conduisant généralement à des déformations admissibles pour les ouvrages construits. 
Seules des conditions de déformations très sévères (en l'occurrence très petites) amenaient à entreprendre de tels calculs en déformation, souvent complexes et revenant finalement à choisir des coefficients de sécurité plus élevés que ceux habituellement utilisés.

A l'inverse, l'estimation de grandes déformations s'avère nécessaire, ne serait-ce que d'un point de vue économique. Ainsi, il est utile de prévoir le volume de matériaux consommé par le tassement d'un remblai construit sur sol compressible, ce qui permet d'en chiffrer à l'avance le coût, indispensable à l'estimation financière du projet.

Cette nouvelle orientation dans la justification des ouvrages n'est pas sans difficultés, et le géotechnicien y est confronté. Il n'est d'ailleurs pas le seul, puisque le sol, son matériau de prédilection, côtoie d'autres matériaux, généralement industriels et donc plus homogènes, mais présentant, eux aussi, des caractéristiques de déformabilité, mieux connues et sujettes à moins de variabilité. Le sol (ou la roche) est ainsi en interaction avec du béton, de l'acier, du bois, des géotextiles. Par ailleurs, la géométrie des ouvrages est rapidement compliquée, et il n'existe que rarement des solutions mathématiques simples, fournissant le résultat pour la situation proposée. Lorsqu'elles existent, elles le sont pratiquement toujours dans le cas de modèles simples de comportement de sol homogène.

Aussi des compromis sont-ils à trouver pour dimensionner les ouvrages, dans le cadre où cela est maintenant exigé. La première préoccupation du géotechnicien est de parvenir à un modèle géologique, dont il établit les propriétés géométriques et géotechniques. Il peut ensuite adopter des lois de comportement simples, n'exigeant qu'un nombre très réduit de paramètres; si l'ouvrage géotechnique est simple et si l'on a la chance de disposer d'une solution mathématique, il pourra résoudre simplement le problème de déformation posé.

Mais le géotechnicien peut aussi tenir compte d'un comportement réel plus complexe des matériaux en présence, surtout pour le sol, et procéder à des calculs a priori plus aptes à rendre compte du comportement réel de l'ouvrage.

Les paramètres de ces modèles restent à déterminer. Dans le premier cas, le paramètre est souvent unique; c'est un module, de type élastique par exemple, avec une hypothèse sur la valeur du coefficient de Poisson. Dans le second, ce seront plusieurs paramètres à fixer suivant les lois de comportement adoptées, avec toutes les difficultés inhérentes à leur mesure et au prélèvement correct d'échantillons de sol ou à la réalisation d'essais en place.

Cet article veut rappeler les définitions des différents modules rencontrés en génie civil, plus spécialement en géotechnique, et la manière dont ils sont mesurés et utilisés. Il existe, en effet, un nombre important d'essais et de procédures pour les déterminer, et il faut reconnaitre que de ce point de vue le géotechnicien a le choix.

Mais des erreurs grossières sont courantes dans l'appréciation des tassements, du fait d'une méconnaissance de la signification réelle du terme de module et d'une mauvaise estimation et utilisation de sa valeur. Ces erreurs sont quelquefois lourdes de conséquences pour des ouvrages construits, en termes, soit de désordres, soit, au contraire, de sécurité surabondante et donc inutile. C'est un des buts recherchés ici que de contribuer à limiter de tels constats.

\section{Le matériau élastique et les essais élémentaires}

Depuis l'Antiquité, jusqu'au Xvir siècle, les corps solides étaient assimilés à des solides indéformables (le solide d'Euclide); cette grande simplification n'empêche d'ailleurs pas encore aujourd'hui d'aborder et de justifier le comportement à la rupture; on considère que des blocs indéformables glissent les uns sur les autres le long de lignes de "rupture» (ou de moindre résistance), où la résistance ultime au cisaillement est atteinte.

Le matériau élastique est celui qui permet le mieux de représenter ce qu'est un module de déformation. Le corps parfaitement élastique, à comportement linéaire et isotrope, est le plus simple, ayant des propriétés identiques dans toutes les directions de l'espace. Ce n'est pas toujours le cas, puisque le bois, par exemple, du fait de sa croissance, ne possède pas les mêmes propriétés verticalement et horizontalement. Il en est de même d'autres matériaux industriels du fait de leur mode d'élaboration.

Pour le sol, dans le domaine limité des contraintes appliquées où l'on peut admettre que l'élasticité régit le comportement, il peut en être également de même; ainsi pour des formations alluvionnaires, le mode de dépôt par couches horizontales a conduit le plus souvent à un état mécanique anisotrope ; ce n'est pas le cas, au contraire, de massifs ayant subi des forces tectoniques ou issus de coulées magmatiques et pour lesquels les propriétés sont encore plus anisotropes.

L'élasticité correspond à la propriété d'un corps de retrouver intégralement sa géométrie initiale après l'application des efforts qui l'ont déformé. Cette propriété a été mise en évidence expérimentalement au XVI ${ }^{e}$ siècle et décrite en 1676 précisément par un savant anglais, Hooke (1635-1703), qui a laissé son nom à la loi correspondante. Le caractère élastique d’un matériau n'est vrai que dans une plage de contrainte appliquée, qui n'est généralement qu'une fraction, très variable suivant le matériau, de la plage totale des contraintes qu'il faut mobiliser pour atteindre la rupture. Dans la suite du texte, le terme d'élasticité utilisé seul sousentend l'élasticité linéaire et isotrope.

\section{1}

\section{L'essai de compression ou de traction, simple}

La mesure considérée comme la plus simple, du caractère élastique et isotrope d'un solide, consiste à soumettre une éprouvette parallélépipédique ou cylindrique de section $\mathrm{S}$ à un essai de compression simple ou a un essai de traction simple, tel que représenté figure 1.

L'extension de l'élément est alors donnée par $\frac{\Delta \mathrm{L}}{\mathrm{L}}=\varepsilon_{\mathrm{z}}=\frac{\sigma_{2}}{\mathrm{E}}$ et l'allongement par $\Delta \mathrm{L}=\frac{\mathrm{FL}}{\mathrm{ES}}$; par convention $\sigma_{7}$ est négatif en cas de compression, et positif dans le cas de la traction. Cette relation permet donc d'accéder au module d'élasticité E, appelé également module d'Young, du nom d'un médecin et physicien anglais (1773-1829). 
Cette extension longitudinale s'accompagne de déformations latérales qui s'expriment par $\varepsilon_{\mathrm{x}}=\varepsilon_{\mathrm{y}}=-v \frac{\sigma_{z}}{\mathrm{E}}$, où $v$ est le coefficient de Poisson (mathématicien français, 1781-1840), qui est la seconde caractéristique du matériau linéairement élastique. Ces trois relations conduisent à la variation de volume de l'éprouvette donnée par:

$\frac{\Delta V}{V}=\varepsilon_{x}+\varepsilon_{y}+\varepsilon_{z}=\frac{\sigma_{z}}{E}(1-2 v)$.

Ceci montre qu'un dispositif permettant de mesurer la seule variation de volume $\Delta \mathrm{V}$ ne permet pas d'accéder directement au module; pour $v=0,5$, valeur maximale du coefficient de Poisson, $\Delta V$ est nul, le sol est déformable mais est incompressible.

C'est donc la mesure simultanée de $\varepsilon$, et $\varepsilon$, qu'il y a lieu d'envisager ; elle présente cependant quelques difficultés opératoires liées au fait que l'effort $F$ est généralement appliqué par l'intermédiaire de plaques de contact, rigides, aux extrémités de l'éprourvette; cela empêche, du fait du frottement, la libre extension radiale à ce niveau et entraîne une déformation dite en " tonneau ». L'utilisation de graisse, téflon, permet de pallier cette difficulté.

\section{2}

\section{L'essai de compression triaxiale}

L'essai précédent, simple dans son principe n'est pas facilement réalisable dans le cas d'un sol, pour lequel les éprouvettes seront de toute manière cylindriques, car, très souvent, prélevées par carottage ou reconstituées telles. En outre, celles-ci sont souvent incapables de se maintenir stables; c'est le cas des sols pulvérulents et des sols très peu cohésifs. Aussi a-t-il été développé des essais particuliers que sont les essais triaxiaux, où le maintien de l'éprouvette est assuré par une étreinte de confinement sur toutes les faces; il est ensuite procédé à l'application en compression de l'effort F évoqué.

En restant dans le cadre strictement élastique, sur un élément toujours parallélépipédique, le système de contraintes appliquées se réduit à $\sigma_{x}, \sigma_{y}, \sigma_{x}$; l'application du principe de superposition, applicable à l'élasticité, conduit aux trois extensions suivantes :

$$
\begin{aligned}
& \varepsilon_{\mathrm{x}}=\frac{1}{E}\left[\sigma_{\mathrm{x}}-v\left(\sigma_{\mathrm{y}}+\sigma_{z}\right)\right] \\
& \varepsilon_{\mathrm{y}}=\frac{1}{E}\left[\sigma_{y}-v\left(\sigma_{z}+\sigma_{\mathrm{x}}\right)\right] \\
& \varepsilon_{\mathrm{z}}=\frac{1}{E}\left[\sigma_{z}-v\left(\sigma_{\mathrm{x}}+\sigma_{y}\right)\right]
\end{aligned}
$$

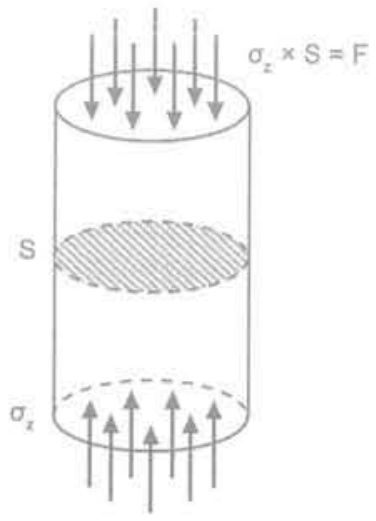

FIG.1 Essai de compression simple, Compression uniaxial test.
Pour une éprouvette cylindrique $\sigma_{\mathrm{y}}=\sigma_{\mathrm{x}}=\sigma_{\mathrm{f}}$ (contrainte radiale), ce qui simplifie les expressions cidessus. La variation de volume est $\frac{\Delta V}{V}=\frac{1-2 v}{E}\left(2 \sigma_{\mathrm{r}}+\sigma_{z}\right)$,
linéaire avec $\sigma_{z}$.

La mesure de deux grandeurs ( $\varepsilon_{z}$ et $\varepsilon_{\mathrm{r}}$ par exemple) permet d'accéder aux deux caractéristiques élastiques E etv.

\section{L'essai de type œdométrique}

On peut imaginer un autre type d'essai, à géométrie simple, qui consiste à enfermer un échantillon de matériau élastique d'épaisseur $\mathrm{L}$ dans une boite totalement indéformable latéralement, cylíndrique ou cubique et simplement ouverte à sa partie supérieure, où l'on applique par l'intermédiaire d'un piston rigide, un effort F.

Dans ce cas particulier, les déformations radiales sont évidemment empéchées et $\varepsilon_{\mathrm{y}}=\varepsilon_{\mathrm{x}}\left(\right.$ ou $\varepsilon_{\mathrm{r}}$ ) $=0$; on trouve facilement dans ces conditions $\frac{\Delta \mathrm{L}}{\mathrm{L}}=\varepsilon_{2}=\frac{\sigma_{2}}{\mathrm{E}} \frac{(1+v)(1-2 v)}{1-v}$, qui ne permet d'accéder à $E$ que si l'on connaît v. On constate d'ailleurs que si $v=0,5$ (matériau incompressible), $E_{2}$ est nul, le module n'est pas mesurable.

Les deux essais réalisables en laboratoire sur les sols, triaxial et à déformation latérale empèchée, n'ont jamais été mis en œuvre pour les matériaux comme l'acier, ayant été à l'origine de l'élasticité expérimentale et des lois de comportement qui en ont résulté. L'acier se prête particulièrement bien à l'essai très simple de compression, et plus précisément de traction.

Comme il a déjá été dit pour l'essai triaxial, c'est la mécanique des sols qui a été à l'origine de son développement, comme celui à déformation empêchée, où l'on reconnaît le principe de l'essai œdométrique, dont il sera parlé plus loin, avec les conditions particulières de son utilisation adaptée aux propriétés du sol.

\section{4}

\section{L'essai de cisaillement simple (ou de distorsion)}

Un autre type de déformation, angulaire ou de distorsion, peut affecter un matériau élastique, et l'illus-

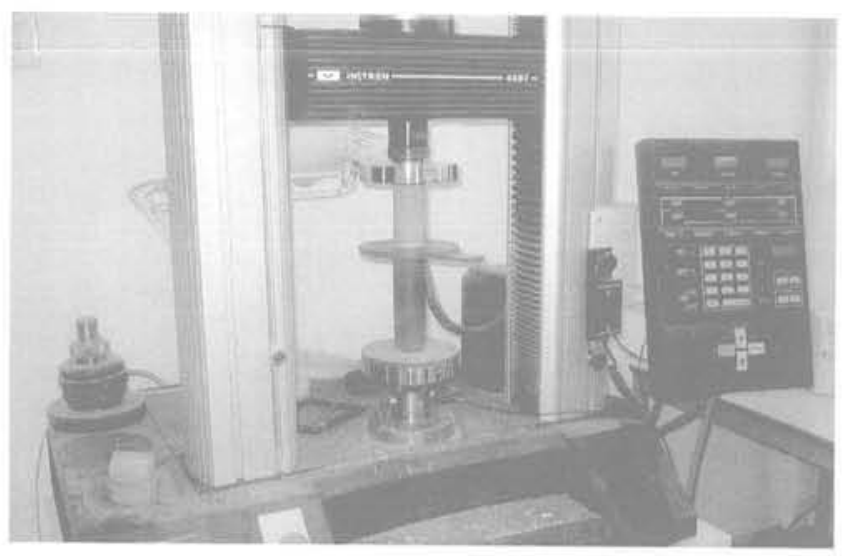


tration la plus simple qu'on peut en donner est la suivante, représentée figure 2.

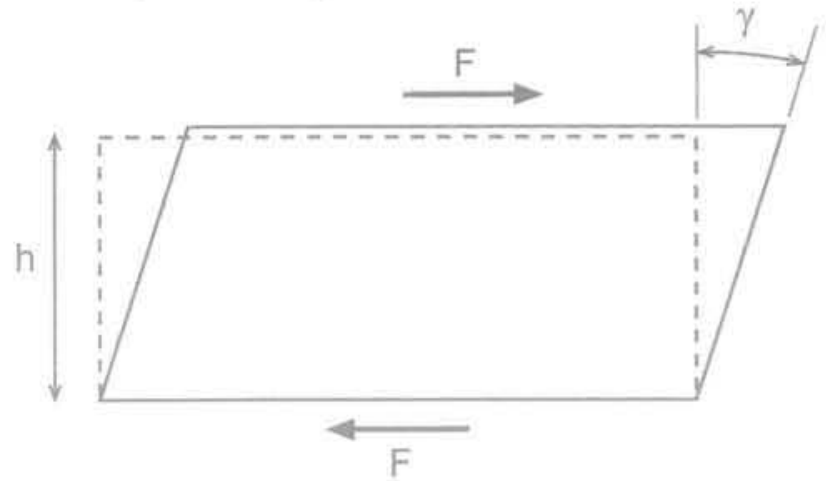

FIG.2 Distorsion lors d'un cisaillement simple. Distorsion in shearing test.

Si l'on applique à la surface S d'un parallélépipède rectangle une force $F$ tangentielle, les différentes couches élémentaires infíniment petites glissent les unes sur les autres comme les feuilles de papier d'une pile ; la hauteur h ne change pas, seules les arêtes normales au plan d'application de la force tournent d'un angle $\gamma$, petit, que l'on peut considérer en première approximation lié à la force par la relation $\gamma=\frac{1}{G} \frac{F}{S}=\frac{\tau}{G}$. où $\mathrm{G}$ est appelé module de cisaillement, module de glissement où module de rigidité.

On démontre que $G$ est lié à $E$ et v par la relation $G=\frac{E}{2(1+v)}$.

Ainsi, dans le cas très général d'un petit cube élémentaire au sein d'un solide linéairement élastique et isotrope, la déformation, produite par trois composantes normales et trois composantes tangentielles, résulte des trois compressions (ou extensions) superposées à trois efforts tangentiels auxquels on peut réduire les contraintes. La justification des ouvrages élastiques repose sur la vérification en tout point du respect des propriétés élastiques, ce que les solutions analytiques ou les méthodes numériques permettent de faire en même temps qu'elles fournissent la valeur des déformations résultantes affectant l'ouvrage.

\section{5}

\section{L'essai d'expansion cylindrique}

La justification des conduites (tubes minces ou épais), parcourues par des fluides sous pression, est aisée avec l'élasticité qui permet de déterminer les contraintes dans le matériau constituant le tube.

Appliqué à un massif élastique indéfini dans lequel une cavité cylindrique de section droite circulaire et de volume $\mathrm{V}_{0}$ est mise sous la pression $\mathrm{p}$, le calcul fournit le volume $V$, donné par $V=V_{0} e^{\frac{p-p}{G}}$, où $p_{0}$ est une pression initiale isotrope au sein du massif. Cette relation n'est pas linéaire ; elle permet de déterminer G et s'écrit, si $\left(p-p_{0}\right) / G$ est petit, $V=V_{0}\left(1+\frac{p-p_{0}}{G}\right)$, soit $G=V_{0} \frac{p-p_{0}}{V-V_{0}}$

Ce type de sollicitation est donc apparemment très simple; il conduit à des déformations insignifiantes dans le cas des tubes évoqués plus haut oủ le problème étudié est en réalité celui des contraintes développées. Appliqué au sol dans un forage, ce principe constitue l'un des moyens de mesure du module de cisaillement, et indirectement du module $E$, avec, cependant, les difficultés liées entre autres au comportement rhéologique du sol ; le pressiomètre, qui a maintenant cinquante ans, est l'outil développé à cet effet pour les sols et les roches très altérées.

Cet essai sera évoqué plus loin, avec son utilisation pour les massifs de sol, Le dilatomètre, basé sur un principe identique, a été développé pour les roches tendres ou fissurées.

\section{6}

\section{Essai de chargement statique par une plaque}

Cet essai, consistant à charger la surface du sol à l'aide d'une plaque, a été initialement développé pour calculer les contraintes et les déformations sous les fondations. La solution analytique, en élasticité linéaire et isotrope, donnée en 1885 par le mathématicien français J. Boussinesq (1842-1929), pour une force ponctuelle P en surface d'un massif semi-infini, a ouvert la voie, la même arnée, pour celle d'une plaque circulaire infiniment souple, puis rectangulaire, posée sur cette même surface.

L'expression la plus couramment utilisée est celle donnant le déplacement de la plaque en son centre. Elle s'écrit pour une plaque infiniment souple, de rayon a, chargeant uniformément la surface du sol sous la contrainte $q, S_{\max }=\frac{2\left(1-v^{2}\right) q a}{E}$, qui est le tassement au centre, sachant que celui-ci n'est pas uniforme et vaut sur le contour, $\frac{4\left(1-v^{2}\right) q a}{\pi \mathrm{E}}$.

Dans la pratique, il s'est avéré qu'il était technologiquement difficile de disposer de plaques réellement

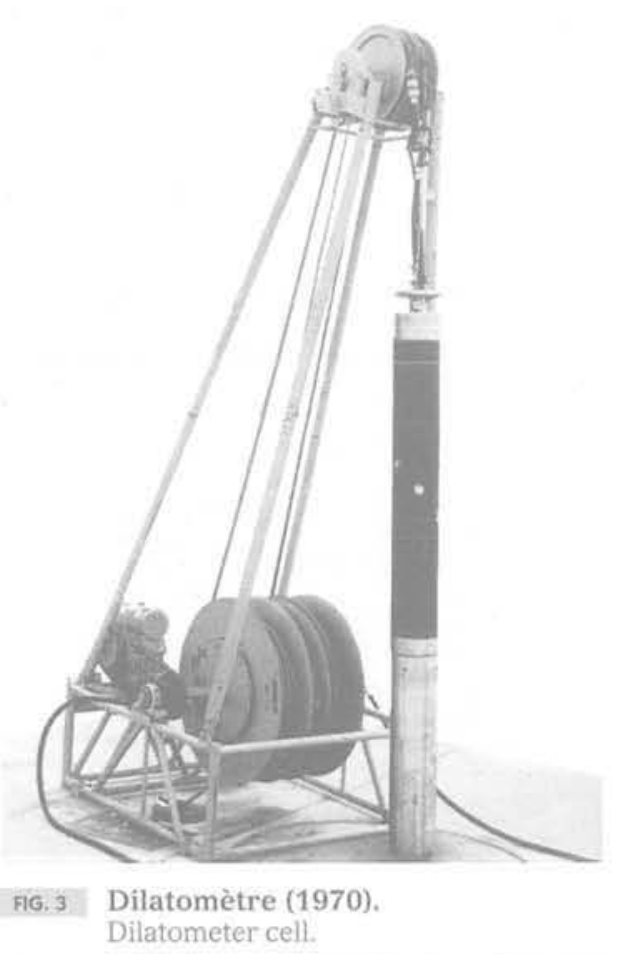




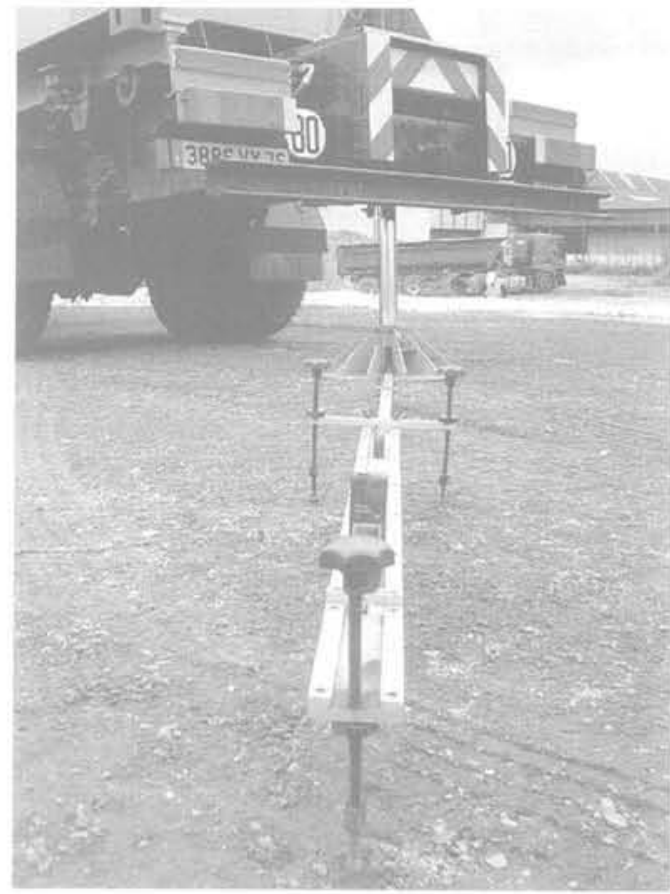

FlG. 4 Essai de chargement à la plaque. Plate bearing test.

souples, pour des rayons a nécessairement limités afin de modérer les efforts à appliquer. Aussi, cette utilisation de plaques souples en France, au début des années soixante, les coussins Claeyssen, du nom de leur concepteur, a-telle été abandonnée à la fin de la décennie.

Elles ont été avantageusement et partout remplacées par des plaques rigides, provoquant un enfoncement constant sous charge, la répartition de la pression n'étant pas, en contrepartie, uniforme, cette dernière étant théoriquement infinie sur le contour. Le tassement, uniforme est alors donné par:

$$
S=\frac{\pi}{2} \frac{\left(1-v^{2}\right) q a}{E}
$$

Cette utilisation de l'essai de chargement à la plaque a connu un essor important avec le développement de la mécanique des chaussées nécessitant la détermination du module đ élastique $x \mathrm{E}$, à défaut de celle de $v$. Le développement des méthodes dites « rationnelles $)$ de calcul de ces structures a fait, en effet, un pas important, pour les besoins de la construction de pistes aéroportuaires militaires, avec la publication en 1943 par le professeur américain de génie civil Burmister (1895-1981) de la solution analytique de chargement du «bicouches (couche de sol de caracteristiques $E_{y}, v_{1}$, sur un massif indéfini de caractéristiques $E_{2}, v_{2}$ ), suivi de celles des multicouches et de toutes les méthodes approchées en découlant. Le besoin est donc né, avec l'analogie entre la charge d'un pneumatique et celle d'une plaque, de moyens de mesures pour accéder à des caractéristiques élastiques.

Cet essai, qui était resté limité à des couches de matériaux non traités, de caractéristiques très modérées, les modules E déduits de l'expression atteignant au mieux $500 \mathrm{MPa}$, a vu récemment son utilisation poussée jusqu'à la mesure de modules de $1500 \mathrm{MPa}$ sur les couches de sol traité au ciment, lors de la construction des pistes nécessaires à l'Airbus A380. Les pressions q mises en jeu atteignent plusieurs $\mathrm{MPa}$, c'est-à-dire des ordres de grandeur comparables à celles transmises par des pneumatiques dont les empreintes ont des dimensions également comparables à celles des plaques utilisées qui sont, pour les plus courantes, de $30,40,50,60$ et jusqu'à $75 \mathrm{~cm}$.

Les modules calculés à partir d'essais de plaques sont des modules sécants, et l'allure d'une courbe de chargement et de cycles déchargement-rechargement est très similaire à celle que présente l'essai triaxial. Il n'est donc pas étonnant que chaque mode opératoire d'essai à la plaque conduise à des valeurs différentes de modules. Les raisons en sont identiques à celles qui seront décrites lors de l'examen de cet essai. On note aussi, du fait de leur utilisation en technique routière, que ce soit pour les couches de chaussées non traitées ou pour les plates-formes de terrassement, que les pressions mises en jeu sont souvent très éloignées des pressions de rupture correspondant à un grand enfoncement de la plaque. Les nombreuses pratiques existant dans les années soixante ont d'ailleurs été largement décrites et commentées (LCPC, 1965). Un besoin d'uniformisation, puis de normalisation, actuellement en cours, est donc indispensable.

Actuellement, en France, l'essai à la plaque est encore utilisé avec deux objectifs : l'un routier, pour le calcul des chaussées, consiste en une exigence de module de l'arase de terrassement ou de la couche de forme, le second, utilisé en bâtiment, pour le dimensionnement des dallages est l'essai Westergaard (du nom du professeur américain d'origine danoise, H.M. Westergaard $\cong 1895-1950$ ) ou son adaptation.

Il est intéressant pour le premier cas, objet d'une norme (Afnor, 2000), d'en mentionner l'évolution sur près de quarante années, tant dans le mode opératoire que dans l'interprétation. L'essai représenté figure 5 consiste, après un léger préchargement destiné à assurer un bon contact entre plaque et sol, à charger par paliers une plaque de $60 \mathrm{~cm}$, d'abord à $0,25 \mathrm{MPa}$, puis après déchargement à zéro, à $0,2 \mathrm{MPa}$. Ces deux boucles successives, qui montrent la non-réversibilité (inélasticité) et le phénomène d'hystérésis, conduisent à définir deux modules sécants appelés $\mathrm{E}_{\mathrm{y}}$ et $\mathrm{E}_{\mathrm{y}}$. Le dernier déchargement, entre 0,2 et $0 \mathrm{MPa}$, permet de définir un module également sécant appelé $\mathrm{E}_{t}$, qualifié à

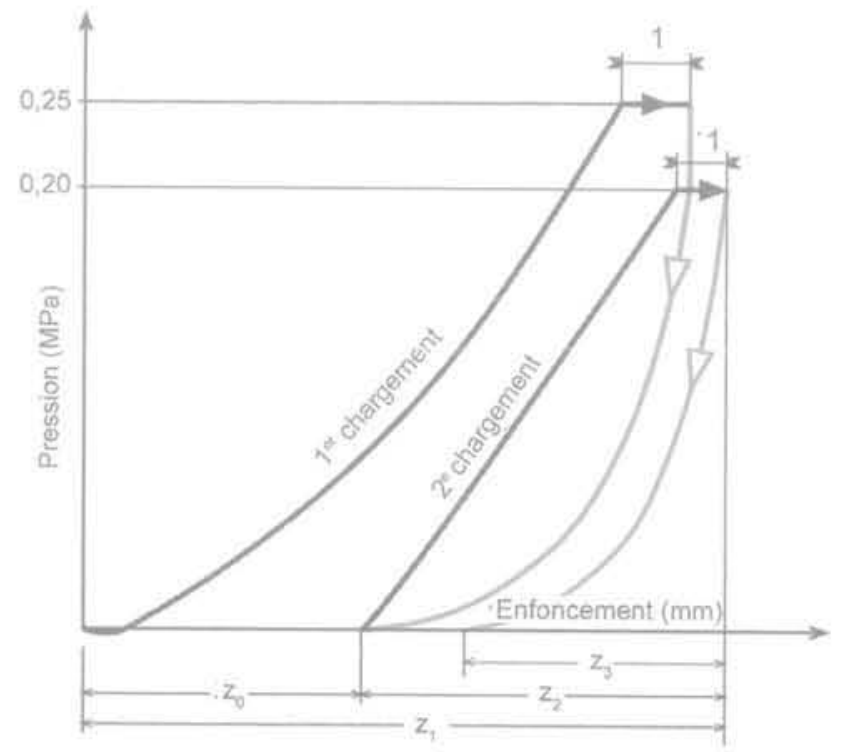

1 ; enfoncements résultant d'une éventuelle attente de la stabilisation.

FIG. 5

Cycle de chargement - déchargement pour l'essai de plaque $\mathrm{Ev}_{2}$. LCPC loading-unloading plate bearing test. 
tort d'élastique, car la réversibilité n'est qu'approchée. Jusqu'à récemment, le rapport $\mathrm{E}_{\mathrm{v} 2} / \mathrm{E}_{\mathrm{v} 1}$ constituait un critère de compactage, la valeur 2 étant contractuellement le seuil au-delà duquel ce compactage était considéré comme mauvais.

S'il est vrai que ce rapport est lié au compactage, ce qu'intuitivement on ressent, le comportement rhéologique du matériau sol, qui est loin d'être élastique, l'explique également en grande partie, et c'est la raison pour laquelle ce critère a été abandonné au profit de la seule considération de la valeur $\mathrm{E}_{\text {w }}$ le premier chargement à $0,25 \mathrm{MPa}$ étant assimilé à une mise en charge préalable du massif.

Tout logiquement, le module sécant $\mathrm{E}_{\mathrm{d}}$ qui peut être tiré de la valeur de déflexion $Z_{3}$ au déchargement fina! est légèrement supérieur à $E_{v_{2}}$.

L'essai de plaque est utilisé pour apprécier la « portance $)$ d'une plate-forme; son utilisation n'a un sens que si le matériau de cette plate-forme (sur une épaisseur suffisante) est identifié, et si son état est connu et supposé pérenne dans le temps. Un tel essai ne constitue donc nécessairement qu'un complément aux contrôles de mise en œuvre. Cette remarque fondamentale, quelquefois perdue de vue, concerne de nombreux essais en place, ou en laboratoire, qu'ils soient statiques ou dynamiques.

Quant à l'essai Westergaard, pour le calcul des dallages, importé des États-Unis, il consiste à mesurer le déplacement e (exprimé en mètres) d'une plaque rigide de $75 \mathrm{~cm}$ de diamètre chargée rapidement sous une pression de $0,07 \mathrm{MPa}$; dans ces conditions, on définit $\mathrm{K}=\frac{7000}{\mathrm{e}}$ exprimé en $\mathrm{kPa} / \mathrm{m}$. C'est le module de Westergaard, qui est une dénomination impropre quant au terme de module. Il est de ce fait appelé également coefficient de réaction.

Si on simplifie la loi de comportement du sol en admettant l'élasticité, on trouve alors la relation $\mathrm{E}_{\mathrm{v} 1}(\mathrm{kPa})=$ $0,55 \mathrm{~K}$; en réalité, la plaque $\varnothing 75$ n'est chargée qu'à 0,07 MPa contre $0,25 \mathrm{MPa}$ avec une plaque $\varnothing 60$. Par suite de la non-linéarité, le comportement est donc plus raide pour l'essai Westerqaard. Par ailleurs, l'influence est légèrement plus forte en profondeur, ce qui, si le massif testé est homogène, contribue à augmenter encore cette raideur, la rigidité du massif de sol croissant avec cette profondeur ; cette influence reste néanmoins modérée ici. On peut considérer dans ces conditions que le coefficient de 0,55 constitue une borne supérieure, la réalité se situant plutôt vers 0,45 à 0,50 .

Ce coefficient $\mathrm{K}$, destiné dans le calcul d'un dallage à dimensionner ce dernier (du moins avant la récente publication du DTU 13.3), y intervient par sa racine quatrième. Il peut être « mesuré $)$ en aménageant l'essai LCPC avec la plaque $\varnothing 60 \mathrm{~cm}$, par une modification du mode opératoire, avec l'adjonction d'un palier de charge intermédiaire, fixé à $0,07 \mathrm{MPa}$. En faisant les mêmes hypothèses dè linéarité que ci-dessus, il vient alors $K=\frac{7000}{\mathrm{e}} \times \frac{60}{75}$.

Pour terminer avec l'essai statique de plaque, il convient d'indiquer qu'il n'est pratiquement jamais utilisé pour dimensionner des fondations. Tout au plus pourraitil être utile pour des semelles superficielles ou quasi superficielles de dimensions transversales d'un ordre de grandeur de celle de la plaque, en veillant cependant à la représentativité de l'essai, réalisé dans des sols naturels de surface, et forcément soumis à de possibles fluctua- tions de leur état. Cette même dernière remarque vaut d'ailleurs aussi pour les autres types d'essais en place exécutés dans les couches superficielles du sol.

\section{1}

\section{Approche des modules par mesures dynamiques}

Dans ce qui a été dit jusqu'à présent, il était admis implicitement que le corps élastique ou non était au repos sous l'action des forces extérieures appliquées : il s'agit donc de problèmes de statique.

Certaines forces, variables, telle une impulsion sinusoïdale ou soudaine, peuvent agir sur le corps élastique et de ce fait lui communiquer un mouvement qui va s'y propager, Les vibrations transmises par les tremblements de terre en constituent un exemple.

Le développement théorique, allié à celui d'une technologie de mesure et d'une analyse de signal fines, permet des approches tout à fait intéressantes et performantes pour la détermination des modules, aux très faibles déformations lesquelles correspondent pratiquement à un comportement élastique des matériaux étudiés.

Ainsi sur une éprouvette prismatique de module $\mathrm{E}$, élancée, la vitesse c des ondes longitudinales de compression, qu'il suffit de mesurer, est liée aux caractéristiques du matériau suivant la relation $C=\sqrt{\frac{E}{\rho}}$ où $\rho$ est la masse volumique du matériau. Ce type de mesure, facile à réaliser, est utilisé pour la détermination des

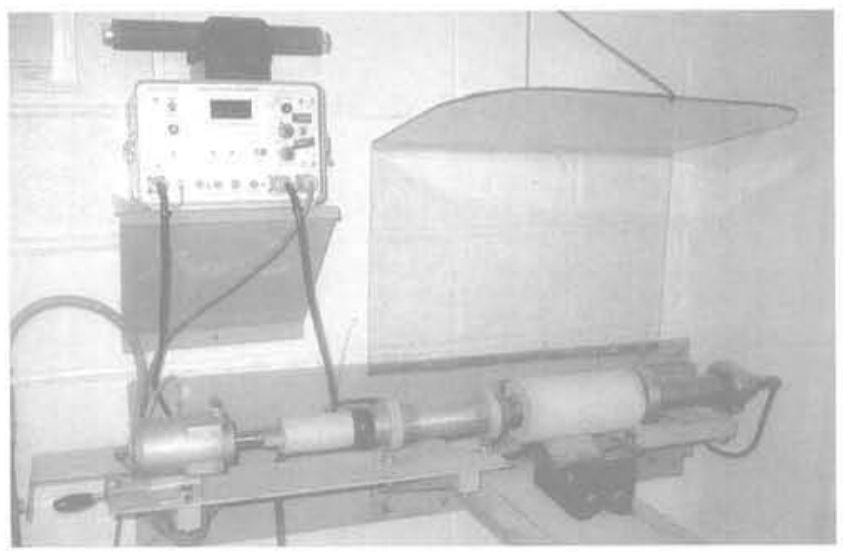

FIG. 6 Mesure de vitesse du son. Soundwave velocity measurement.

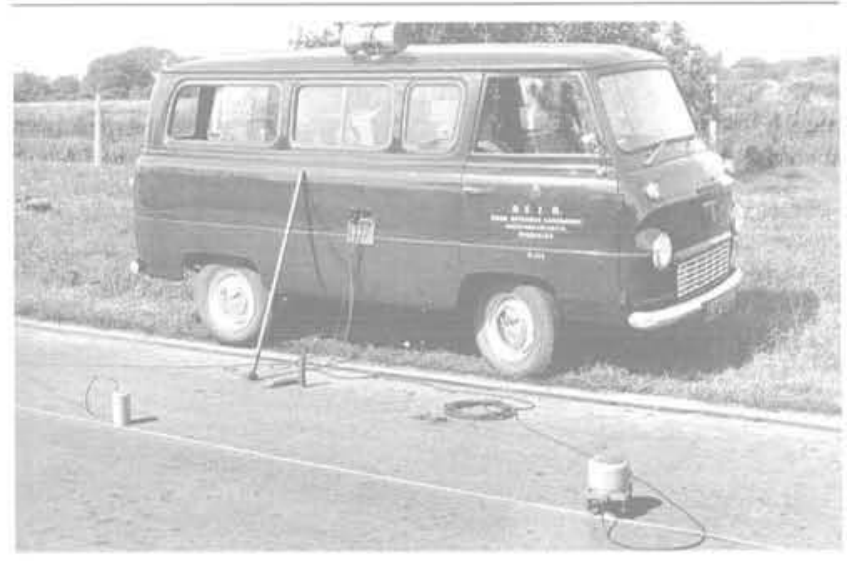

FG.7 Vibreur Goodman. Goodman vibrator 


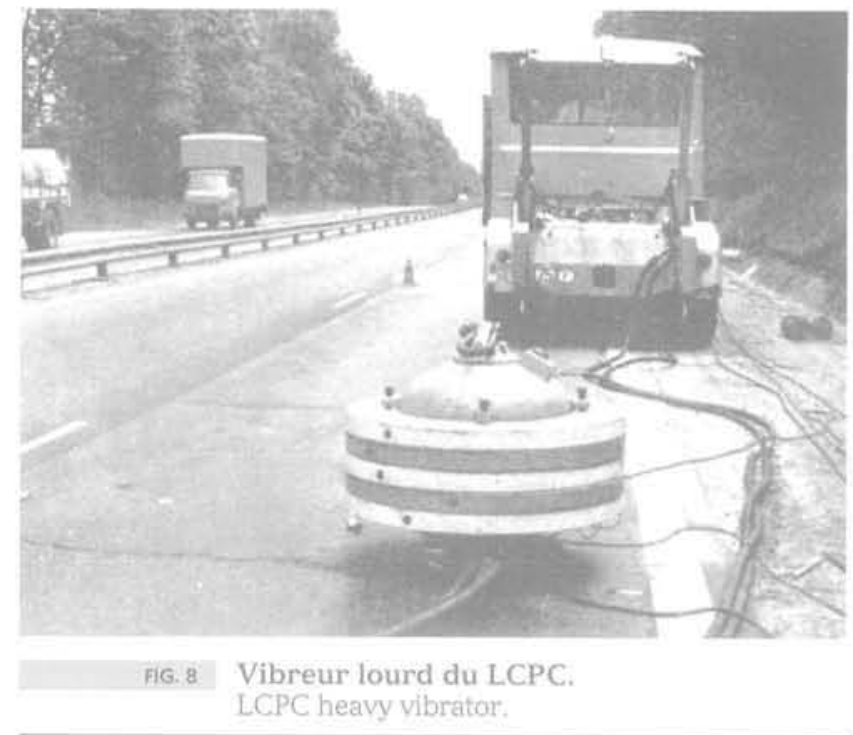

modules élastiques des matériaux traités, utilisés dans le domaine des chaussées ou de la géotechnique routière.

Dans un massif linéairement élastique, où les ondes peuvent rayonner dans toutes les directions, les modes de propagation sont différents, diversifiés et plus complexes que précédemment. Les ondes longitudinales de compression se propagent avec une vitesse $C_{v}$, telle que $C_{1}^{2}=C^{2} \frac{(1-v)}{(1+v)(1-2 v)} ; C_{2}$ peut donc être très différente de $C$ suivant la valeur du coefficient de Poisson.

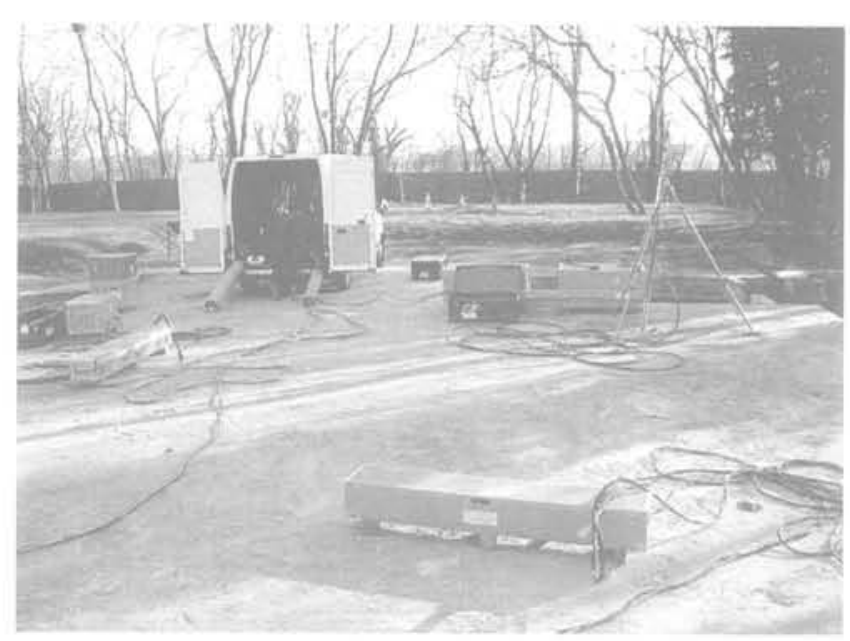

FG, 9 Dispositif cross hole (avec triplet). Cross hole dispositive.

Des ondes transversales de distorsion ou de cisaillement se propagent également qui sont, elles, affectées d'une vitesse $C_{2}=\sqrt{\frac{G}{\rho}}$, où apparait le module de cisaillement $G$; ces ondes sont maintenant couramment exploitées en géotechnique, et permettent d'accéder à la mesure de $\mathrm{G}$ aux très faibles déformations.

Enfin, il faut citer un cas particulier de propagation d'ondes qui se forment à la surface d'un corps solide semi indéfini (surface du sol), par exemple, et appelées ondes de surface ou ondes de Rayleigh, physicien anglais (1842-1919) qui les étudia en 1887. Ces ondes particulières ont une vitesse $\mathrm{C}_{3}$, telle que :

$$
\left(\frac{C_{3}^{2}}{C_{2}^{2}}-2\right)^{4}=16\left(1-\frac{C_{3}^{2}}{C_{1}^{2}}\right)\left(1-\frac{C_{3}^{2}}{C_{2}^{2}}\right)
$$

D'un point de vue pratique, cette vitesse $C_{3}$ reste proche de la vitesse $\mathrm{C}_{2}$, l'écart maximal atteignant $8 \%$. Les ondes de surface ont trouvé une large application, d'abord dans le domaine des structures de chaussées, assimilées à des couches stratifiées posées sur massif indéfini ; les années 60 ont vu se développer la technique, importée de Grande-Bretagne, du vibreur Goodman (LCPC, 1968) puis du vibreur lourd, générant de

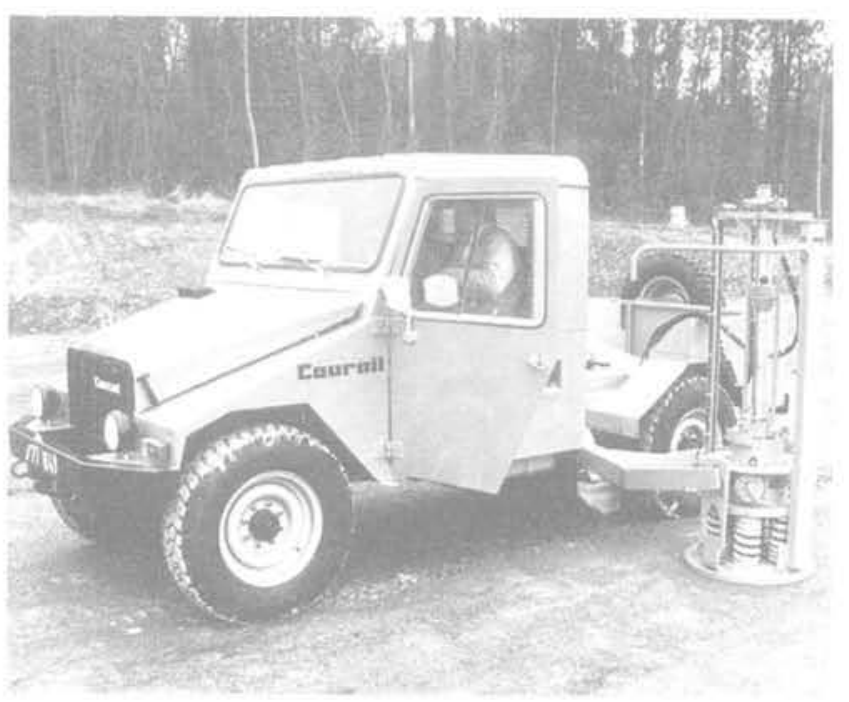

FIG. 10 Dynaplaque 1 (1982). Dynaplaque 1 test.

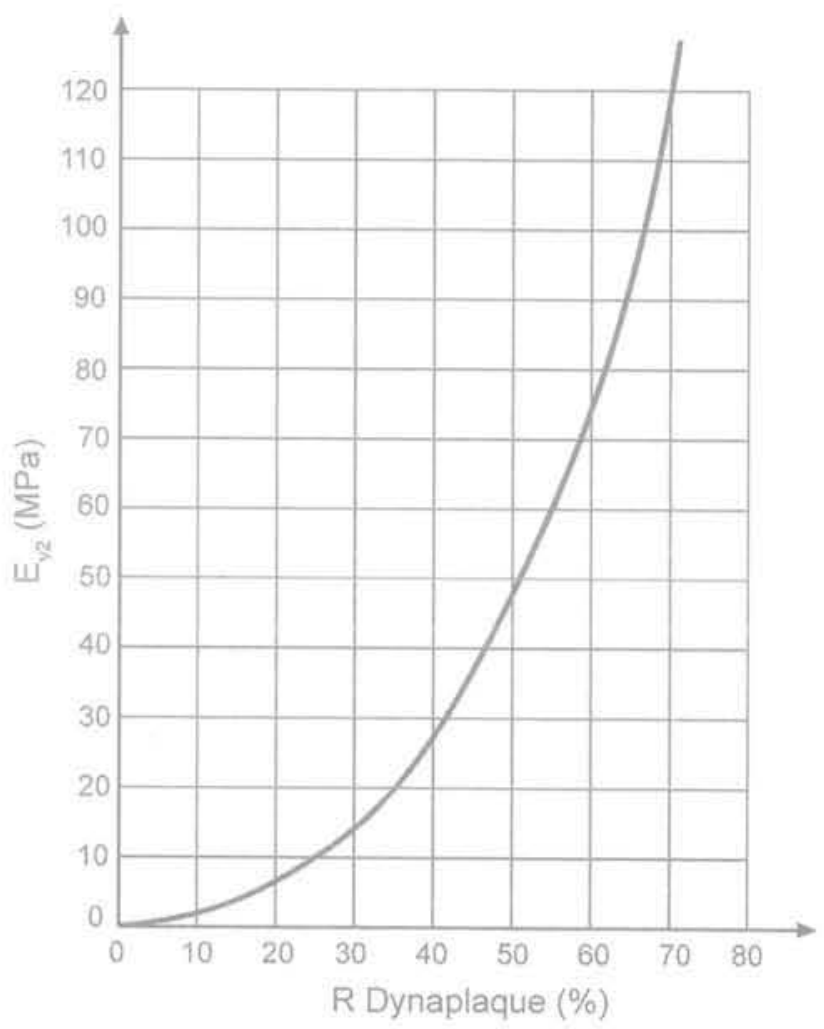

FIG. 11 Dynaplaque de type 1 ; relation expérimentale entre le coefficient de restitution $\mathrm{R}$ et le module $\mathrm{E}_{v 2}$.

Dynaplaque 1 ; experimental relation between restitution coefficient $R$ and $E_{v_{2}}$ modulus. 
telles ondes. Le but recherché consistait à déterminer, là aussi, les modules des couches concernées nécessaires au calcul « rationnel » des chaussées.

Un peu laissées à l'écart, les ondes de surface voient, à l'heure actuelle, un nouveau développement en géotechnique pour apprécier la déformabilité des massifs, de mème que la transmission d'ondes soniques de forage à forage, constitue un autre moyen pour cette détermination. Les techniques de résolution d'analyses inverses (ou rétro-analyses) ont en effet favorisé leur utilisation. On notera les techniques dites cross-hole ou down-hole, ou, plus récemment, le cône

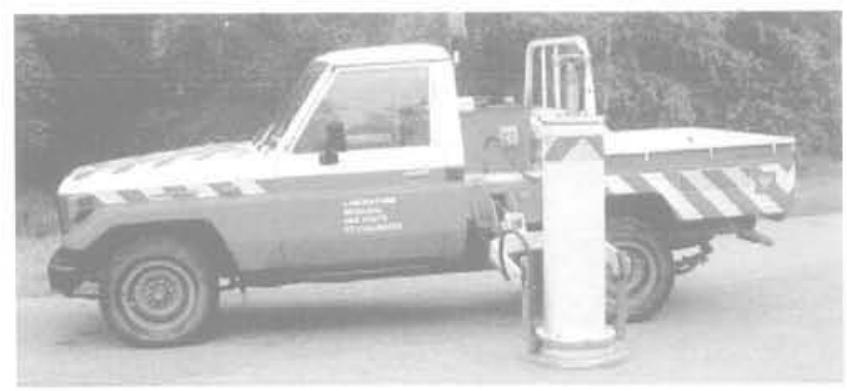

FG. 12 Dynaplaque 2.

Dynaplaque 2 test.

sismique jumelant la pénétration statique et la mesure de vitesse d'ondes sismiques émises.

L'application des moyens d'auscultation dynamique est maintenant opérationnelle en laboratoire sur éprouvettes et permet en particulier des comparaisons avec les modules statiques obtenus eux aussi en laboratoire dans le domaine des petites déformations. A titre d'exemple, il a été montré sur du sable, au moyen d'essais triaxiaux, l'équivalence entre ces modules pour des amplitudes de déformation de l'ordre de $10^{-5}$ (Pham Van Bang, 2004).

L'analyse du choc d'une masse sur un massif (déflexion dynamique) est un second mode de sollicita-

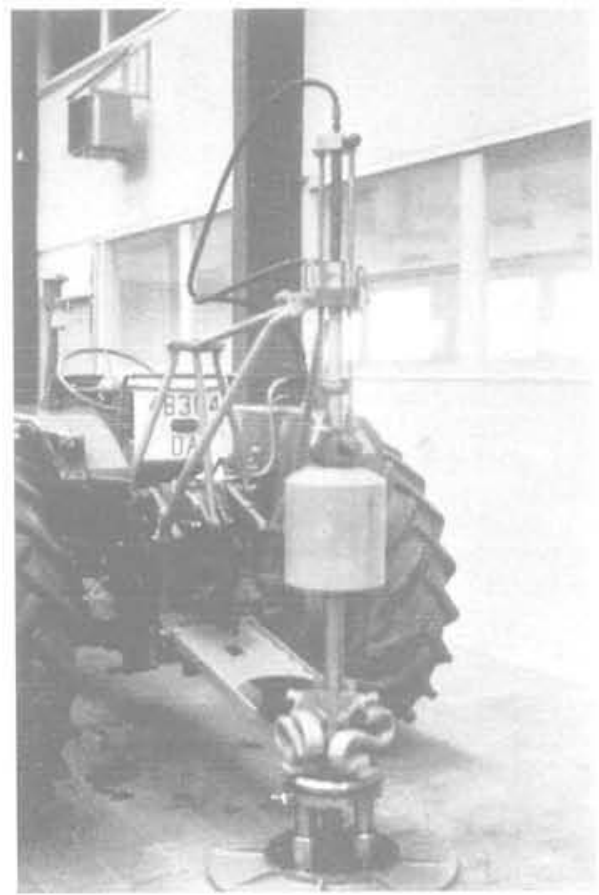

FIG. 13 Déflectomètre à boulet et à “jarret » (1963). Ball deflectometer with "jarret" self damping springs.

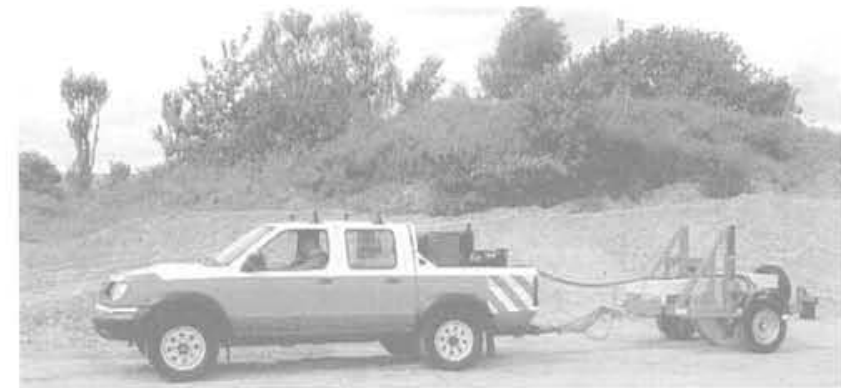

FIG. 14 Portancemètre. "Portancemetre"

tion dynamique de celui-ci. Elle a été mise à profit d'une manière différente, quant à sa traduction en terme de module, avec le développement de la dynaplaque, dite maintenant de type 1 pour la différencier de celle de type 2 qui lui a succédé. La première utilise la hauteur de rebond h d'une masse chutant d'une hauteur h'sur des ressorts fixés à une plaque posée au sol. Le rapport $\mathrm{R}=\mathrm{h}^{\prime} / \mathrm{h}<1$ (coefficient de restitution) est traduit en un module $\mathrm{E}_{\mathrm{v},}$, restitué figure 11, résultant de comparaisons expérimentales effectuées à partir de mesures de modules statiques à la plaque, lors de la mise au point de ce matériel.

Ce matériel est évidemment conçu au niveau des paramètres mécaniques (dimension, charge...) pour que cette restitution ait un sens. Aussi les sollicitations appliquées se rapprochent-elles de celles imprimées par des charges routières standardisées.

La dynaplaque 2 (Chassaing et al., 1995) a bénéficié d'améliorations technologiques. Plus performante, elle permet la détermination d'un module à partir des mesures de la force dynamique appliquée et de la déflexion de la plaque d'appui reposant sur le sol. L'application de l'expression donnant le tassement, rappelée précédemment pour l'essai statique à la plaque, conduit donc à un module qualifié de dynamique. La conception du matériel, comme pour le type 1. conduit à des valeurs de modules similaires à celles pouvant ètre obtenues statiquement; ce sont des modules sécants correspondant à des déformations relatives de l'ordre de $10^{-3}$,

Il est intéressant de rappeler que c'est l'étude et le développement des déflectomètres à boulet (Bretonnière, 1963) qui ont permis l'apparition des dynaplaques, Tous ces matériels sont ponctuels et le besoin d'auscultation en continu, et à plus grand rendement a conduit récemment au portancemetre (Morel, Quibel, 1998) constitué d'une roue vibrante instrumentée tractée. Utilisée pour les travaux de terrassements et de couches de forme, la valeur du module tirée de ce matériel est très bien corrélée avec les modules déterminés à la Dynaplaque ou à l'essai statique classique $\mathrm{E}_{\mathrm{v} \text {. }}$. Sa plage d'utilisation couvre la gamme des modules entre 30 et $300 \mathrm{MPa}$.

\section{2}

\section{Le sol et sa caractérisation élémentaire}

\section{1}

\section{Rappel du comportement d'un sol}

Les méthodes d'interprétation des essais, que l'on vient de décrire et que l'on peut mettre en ceuvre, tant sta- 
tiques que dynamiques, constituent pour la plupart une retombée des analyses théoriques que le développement industriel a nécessitées, au XIXe et au début du XXe siècle (résistance des matériaux). Elles sont issues de l'étude du comportement simple constitué par l'élasticité linéaire (loi de Hooke), et correspondant bien aux propriétés des matériaux alors les plus concernés, tels que l'acier.

Progressivement, et l'essai à la plaque le montre clairement, le sol a été considéré comme un matériau relevant de la mécanique et l'élasticité linéaire lui a été appliquée, même si le caractère approximatif de cette dernière hypothèse est rapidement apparu. Cette approximation reste encore suffisante dans de nombreuses situations dans la mesure où l'on sait rester maitre de son application.

Si le sol était totalement élastique dans les plages de contraintes où on le sollicite, le calcul des déformations en serait grandement facilité, et les résultats des mesures de modules par la mise en œuvre des différents essais décrits seraient cohérents entre eux. Si l'acier comporte une plage étendue de comportement élastique, avant que les phénomènes de rupture locale puis générale n'apparaissent, le sol a la particularité de n'être à peu près élastique que dans une plage de très faibles contraintes, plage au-delà de laquelle les ouvrages le sollicitent très largement.

Le sol diffère profondément du matériau élastique, linéaire et isotrope. Il est composé d'un squelette de particules entourées de plus ou moins d'eau et de gaz. Cet ensemble est caractérisé par un comportement très particulier :

- une absence, ou presque, de résistance à la traction ; - un comportement non linéaire :

- une déformation non réversible;

- une anisotropie le plus souvent orthotrope :

- un effet du temps (fluage, relaxation, fatigue) surtout important pour les sols fins saturés.

Tous ces phénomènes influent sur la détermination des valeurs des modules et plus généralement sur le choix de lois effort-déformation; ils constituent donc une difficulté notable pour le géotechnicien.

La très faible linéarité initiale, puis la non-linéarité qui lui succède trouvent leur explication dans l'examen du sol et sa constitution. On se limitera pour l'instant à un sol granulaire, constitué de grains solides, d'eau et d'air, Les grains de matière sont en contact les uns avec les autres : larrangement est plus ou moins dense et est fonction de la répartition granulométrique et du serrage. Le serrage Iui-même dépend de l'histoire du massif, donc de sa genèse et des charges qu'il a pu subir par le passé.

Plus le poids volumique est élevé, plus l'indice des vides est faible et moins l'ensemble est déformable, car le nombre de points de contact entre les grains augmente. On imagine aisément que, sous de très faibles sollicitations, les contacts entre grains restent approximativement élastiques et les déformations à peu près réversibles ; si les contraintes augmentent, les grains peuvent se déplacer à leurs points de contact, et ils finissent par glisser les uns sur les autres, ce qui conduit à des réarrangements et des déformations de plus en plus importantes. Suivant l'angularité des grains, certains peuvent même être cassés.

Ces déformations sont irréversibles et dites plastiques. Elles ne sont pas instantanées lors de l'application de la charge, ce qui se traduit par ce que l'on appelle le fluage. Dans le cas de la répétition de la charge appliquée, le phé- nomène de fatigue participe avec le temps à ces déformations irréversibles. Fluage et fatigue, dans le cas d'un sol granulaire, restent petits, dans le domaine de contraintes où le sol est sollicité. Il convient également de mentionner les phénomènes de dilatance et contractance: Le long des lignes ou surfaces de cisaillement, qui canalisent la rupture, les mouvements relatifs sol-sol ne se produisent que si, localement, les grains peuvent se déplacer les uns par rapport aux autres. Si le sol est lâche, ces déplacements sont faciles et conduisent à un resserrement local des grains, avec, donc, une diminution locale du volume élémentaire; c'est le phénomène de contractance. Inversement, si le sol est dense, le mouvement ne s'opère que si un désenchevêtrement se produit, accompagné d'une légère augmentation du volume local : c'est le phénomène de dilatance. Il existe donc une densité dite critique où il n'y a pas variation de volume.

La signification et l'interprétation des modules qui sont déduits des essais de laboratoire nécessitent d'introduire le rôle de la nature du sol, qui conditionne le comportement et amène à évoquer le facteur temps. On distingue en effet pour les sols deux comportements radicalement opposés :

- celui des sols granulaires, très perméables, dont les grains supportent immédiatement et intégralement les charges qui sont transmises. Même saturés, la forte perméabilité permet généralement à l'eau interstitielle de se déplacer rapidement dès que des surpressions apparaissent; le drainage de l'eau est en général immédiat :

- celui des sols peu perméables : comportant une matrice constituée de particules extrêmement fines, ils possèdent, pour les argiles, des structures très particulières et variées où les phénomènes électrochimiques jouent un rôle important. Très souvent saturées, avec des gaz dissous ou non, les particules élémentaires sont entourées d'eau dite adsorbée qui leur est indissociable, et baignent dans l'eau dite libre. Leur comportement n'est pas indépendant du temps. A court terme, c'est l'ensemble sol-eau qui encaisse la charge appliquée, mais c'est surtout l'eau qui la supporte et qui est mise en pression ; il n'y a pas de drainage et l'eau ne s'échappe pas. A long terme en revanche, la surpression de l'eau va diminuer et baisser plus ou moins lentement. I y a drainage du sol par expulsion de l'eau jusqu'à reprise complète des efforts par les grains, et annulation de la surpression interstitielle.

C'est la manière dont le sol réagit sous une charge qui intéresse le géotechnicien et dicte le type d'essai, triaxial en particulier qu'il y aura lieu d'entreprendre : réalisé au laboratoire, il demande à ce que les éprouvettes de sol soient préalablement saturees,

Les sols fins ont un comportement beaucoup plus complexes que les sols granulaires et leur étude, l'interprétation des essais et de leur comportement tant en laboratoire qu'en place s'avère évidemment plus délicate.

Très souvent, les désordres qui affectent les ouvrages géotechniques (semelles, pieux, micropieux, tunnels, soutènements) proviennent de déformations trop importantes du sol; la rupture peut même apparaître. Le sol se trouve soit cisaillé le long de surfaces au sein du massif où il est le plus fortement sollicité, soit très comprimé.

On construit donc les ouvrages géotechniques avec des sécurités suffisantes, en deçà desquelles il n'y a pas de risque ; néanmoins, il faut s'assurer que les déformations restent acceptables pour la construction supportée, laquelle peut être extrêmement sensible. 
On entrevoit et on sépare deux modes différents de champs de contraintes dans le sol. Le premier dit déviatorique entraîne des déformations liées majoritairement à du cisaillement, le second dit sphérique considère des déformations de compression. Ces deux modes, dénommés et utilisés par Louis Ménard dans le calcul des tassements, cohabitent le plus souvent.

Cette étude de la déformation du sol, menée jusqu'à la rupture ou jusqu'à de très grandes déformations, peut être simulée en laboratoire ou en place. Il arrive qu'elle soit réalisée en vraie grandeur, c'est le cas par exemple d'un essai instrumenté de chargement d'un pieu.

Les deux essais de base utilisés au laboratoire pour la mesure de la déformabilité des sols sont l'essai triaxial et l'essai cedométrique, déjà évoqués dans le cadre strict de l'élasticité linéaire, et qui correspondent aux deux aspects précédents du comportement, déviatorique et sphérique.

\section{2}

\section{L'essai triaxial}

Dans cet essai, une éprouvette cylindrique de sol, initialement confinée en laboratoire sous une contrainte isotrope $p_{\text {, }}$, est progressivement soumise à une surcontrainte verticale $q$, la contrainte $p_{1}$ restant constante

Les déformations qui apparaissent sont de deux types. Sous la contrainte initiale de confinement $p_{2}$. elles sont de compression, avec diminution de volume. Lors de l'application de la surcontrainte q, provoquant le cisaillement, c'est une augmentation de volume, qu'une légère diminution éventuelle, liée à la densité du matériau, peut précéder.

La plage des contraintes utilisées dans le dimensionnement d'ouvrages est généralement de l'ordre du tiers ou la moitié de la contrainte de rupture q. La figure 15, extraite avec les commentaires qui l'accompagnent, d'un article récent (Mestat, Reiffsteck, 2002) illustre la diversité des modules que l'on peut définir à partir de la courbe effort-déformation obtenue, compte tenu de la non linéarité et de la non élasticité des sols.

Pour de très faibles déformations, on peut adopter un module initial $\mathrm{E}_{\text {inn }}$ pouvant être considéré comme élastique, qui correspond à des déformations relatives inférieures à $10^{-5}$. Le module sécant $\mathrm{E}_{\text {sec }}$ est essentiellement variable avec le niveau de contrainte $q$ atteint, et on comprend immédiatement les difficultés du calcul en déformation.

A titre indicatif, l'ordre de grandeur courant des déformations des ouvrages en service se situe vers $10^{-2}$ à $5 \cdot 10^{-3}$. Ainsi le tassement d'un pieu ou d'une semelle correctement exécutés et dimensionnés est d'environ 0,005 à 0,01 fois leur diamètre ou largeur.

Le module cyclique, $E_{\text {cyù }}$ représenté figure 17, gouverne la déformation lors d'un cycle déchargementrechargement. Les valeurs du module cyclique sont très variables et dépendent principalement de deux paramètres : le niveau de contrainte maximale $\mathrm{q}_{\mathrm{M}}$ atteint et l'amplitude $\Delta q$ de la contrainte durant le cycle.

Du fait de la non-réversibilité des déformations plastiques apparues lors du premier chargement, la courbe de décharge est très raide, et le module cyclique, très largement supérieur au module sécant, se rapproche du module initial, tout en lui restant inférieur.
Sous des cycles répétés comme la figure 17 le schématise, la déformation augmente par suite de l'accumulation des microdéformations irréversibles plastiques au sein du milieu granulaire. L'amplitude des déformations atteinte après $\mathrm{N}$ cycles peut s'écrire $s(\mathrm{~N})=\mathrm{s}(\Delta \mathrm{q})(1+\mathrm{A} \operatorname{In} \mathrm{N})$ avec $\mathrm{s}(\Delta \mathrm{q})=\frac{\Delta \mathrm{q}}{\mathrm{E}_{\text {cycl }}}$ où $\mathrm{A}$ est un coefficient qui croit avec $\mathrm{q}_{\mathrm{M}}$.

La figure 15 peut également correspondre aux résultats d'un essai drainé, qui sera de réalisation rapide s'il s'agit de sol pulvérulent ou au contraire lente pour des sols très fins saturés. On obtient alors des modules drainés notés E'. Un essai rapide, non drainé, sur matériau fín saturé conduit à des modules non drainés $E_{\text {, }}$ On conçoit bien, étant donné les mécanismes mis en jeu lors du drainage avec un départ d'eau, que $E^{\prime} \leq E_{i}$; nous reviendrons plus loin sur les relations qui les lient (on a $E^{\prime}=E_{n}$ pour des sols très perméables, granulaires)

Entre ces deux situations extrêmes de sols très perméables et quasi imperméables, les sols non saturés, doivent faire l'objet d'une attention particulière en ce qui concerne leur état hydrique, dont il faut s'assurer du caractère permanent ou non. La réalisation d'essais triaxiaux sur des prélèvements en l'état est toujours possible, mais les paramètres qui en sont issus, et les modules en particulier, sont susceptibles de ne caractériser le sol qu'à un moment donné, dans un état donné.

Si l'on souhaite dans les calculs d'ouvrages adopter une loi de comportement conforme aux résultats de la figure 15, le modèle est compliqué, le plus simple utilisé comportant sept paramètres (loi élastoplastique, dite avec écrouissage) qu'il faut déterminer. Ces types de modèles sont introduits dans des codes de calcul aux éléments finis ou différences fïnies.
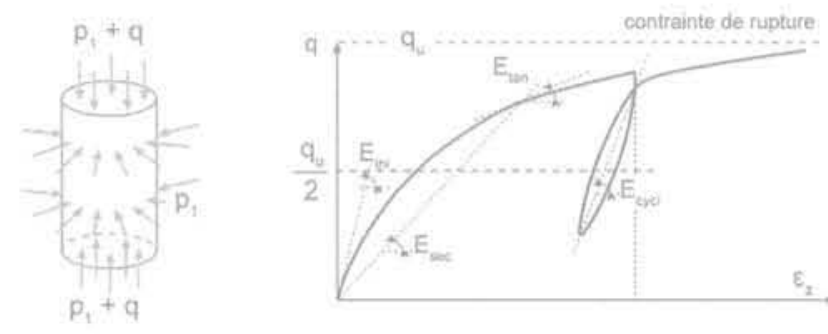

FG. 15 Définition des modules dans l'essai triaxial. Definition of moduli in triaxial test.

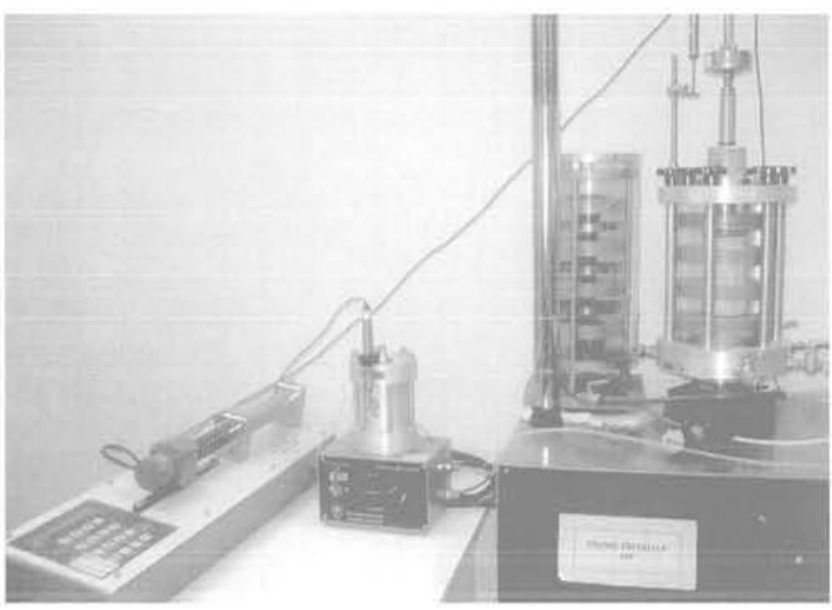

FIG. 16 Appareil triaxial.

Triaxial apparatus. 
Cette figure 15 peut être simplifiée pour des applications pratiques. La figure 18 montre par exemple une loi dite élastoplastique parfaite (modèle de Mohr-Coulomb) comportant une partie linéaire, caractérisée par un module élastique E, suivie d'un palier plastique limité par $q_{4}$, dont la valeur est liée aux caractéristiques $\varphi^{\prime}$ et $c^{\prime}$, s'il s'agit de conditions drainées (la cohésion c' est nulle dans le cas de matériau totalement pulvérulent; on notera $E=E^{\prime}$ ), ou $c_{u}$, cohésion non drainée (et on notera $E=E$ ) s'il s'agit de conditions non drainées pour les sols fins. La valeur de $\mathrm{E}$ à rentrer dans le modèle (sans parler du coefficient de Poisson v) résulte d'un compromis avec le comportement réel ; il s'agira d'un module sécant moyen tiré de la figure 15. Cinq ou quatre paramètres sont indispensables : $E^{\prime}, v^{\prime}, c^{\prime}$ et $\varphi^{\prime}$ et $\psi$, angle de dilatance lié au mécanisme de mème nom déjà évoqué (on adopte souvent $\psi=0$ ), ou $E_{u}, v_{u}, c_{u}$ et $\psi$.

Nous avons indiqué que l'éprouvette triaxiale était confinée sous une contrainte isotrope $p_{1}$. Le choix de cette contrainte n'est pas indifférent de la profondeur à laquelle on prélève l'échantillon de sol. Il s'agit, après extraction de l'échantillon, de recréer avant l'essai proprement dit, les conditions de contraintes effectives auxquelles il était soumis dans le sol. Dans un essai drainé, lobtention des caractéristiques de résistance drainées (ou effectives) c' et $\varphi^{\prime}$ nécessite plusieurs essais élémentaires sous des contraintes isotropes différentes. Aussi, à cette fîn, le choix pour un même élément de sol, situé à la profondeur $z_{2}$, de trois contraintes $p_{3}>p_{2}>p_{4}$ amène à déterminer trois valeurs de module du sol attribuées aux profondeurs correspondantes $z_{2}, z_{2}$ et $z_{1}$. On peut donc avec un essai triaxial complet, ce qui demande trois ou quatre échantillons, en déduire les modules à des profondeurs différentes. Le module croît avec la profondeur.

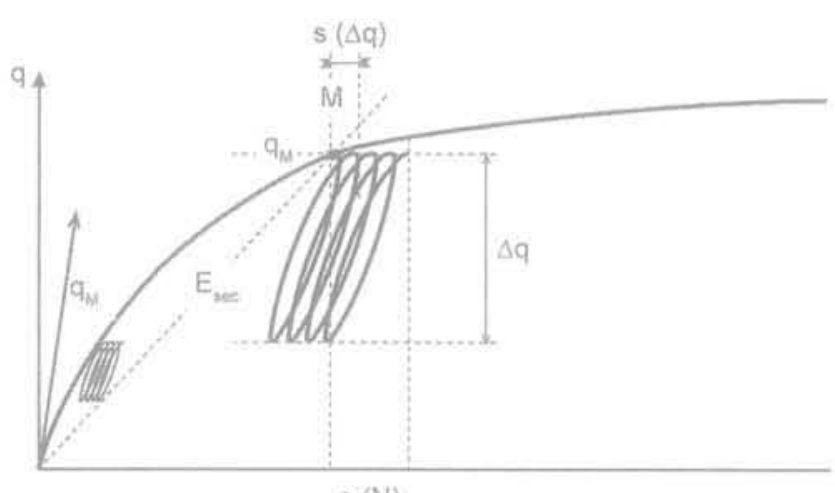

$s(N)$

Fig. 17 Chargements répétés à l'essai triaxial. Cyclic triaxial test.

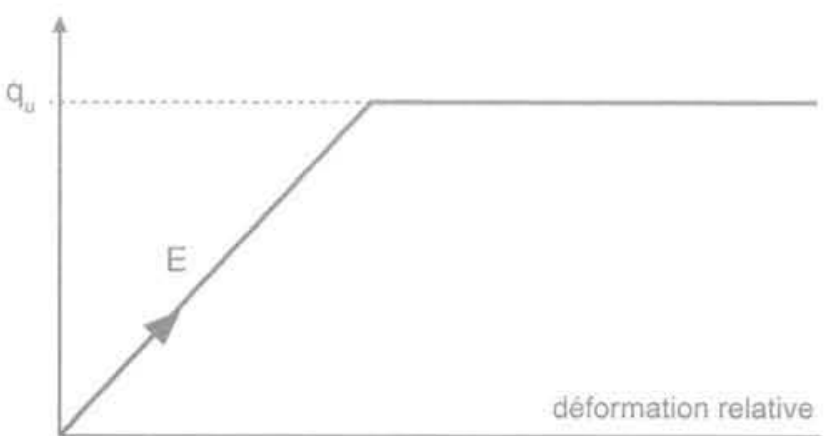

FIG. 18 Loi élastoplastique de Mohr-Coulomb. Mohr-Coulomb elastoplastic behaviour.
Pour un sol fin, dans un essai non drainé, ou rapide, qui conduit à déterminer la cohésion non drainée $\mathrm{c}_{u}$, la courbe contrainte-déformation reste la même quelle que soit la pression de confinement p, si l'on n'autorise pas le drainage préalable avant le cisaillement rapide. Par contre, si, sous différentes étreintes $p$, on permet le drainage complet et que l'on cisaille ensuite rapidement, on peut estimer, comme précédemment, l'augmentation avec la profondeur de la cohésion non drainée $c_{u}$, et du module $E_{\text {. }}$ L'essai correspondant est l'essai consolidé non drainé.

Durant le drainage sous l'étreinte isotrope $p_{1}$ l'échantillon subit une diminution de volume par compression, conduisant à des caractéristiques nouvelles déterminées lors du cisaillement rapide.

Une illustration simple et indirecte de l'augmentation du module avec les contraintes de confinement, et donc avec la profondeur dans le massif, est celle de deux paquets de café ; le premier, rempli simplement à l'air ambiant, se déforme très facilement sous une légère pression en tête : le second a été rempli sous vide, il subit donc la pression atmosphérique et la même légère pression ne le déforme pas car il a acquis une très forte rigidité. On constate aussi que son volume est légèrement plus faible que le précédent, son poids volumique étant légèrement plus élevé. On voit donc, que le module drainé est essentiellement variable, pour un sol donné, en fonction de son état et des contraintes auxquelles on le soumet.

\section{8}

\section{L'essai œdométrique}

L'codomètre est un matériel de laboratoire, mis au point pour les besoins de la mécanique des sols, particulièrement adapté à l'étude des tassements des sols fins et qui permet d'aborder plus simplement que le triaxial la relation entre module drainé et contraintes (ce matériel a initialement été utilisé pour la mesure des gonflements, d'où son nom),

C'est un essai à géométrie simple, qui consiste à enfermer un échantillon d'épaisseur L (quelques centimètres) dans une boite indéformable latéralement, cylindrique ou cubique et simplement ouverte à sa partie supérieure, où l'on applique un effort $F_{\text {y }}$ par l'intermédiaire d'un piston rigide ou par pression pneumatique. C'est un essai où les contraintes appliquées sont plutôt de mode sphérique, entrainant surtout des efforts de compression.

Dans cet essai, les déformations radiales sont empêchées et $\varepsilon_{y}=\varepsilon_{y}\left(\right.$ ou $\left.\varepsilon_{y}\right)=0$. Si l'on suppose le matériau élastique on trouve alors, comme dit en I, que :

$$
\varepsilon_{2}=\frac{\Delta L}{L}=-\frac{\sigma_{2}}{E} \frac{(1+v)(1-2 v)}{(1-v)} \text {, cc qui ne permet d'accé- }
$$

der à $E$ que si $v$ est connu ; en particulier si v se rapproche de 0,5 , ce qui est le cas du milieu incompressible, le module E, alors apparent, tend vers l'infini.

Cette configuration est précisément celle rencontrée lors de la mise en place de remblais ou radiers de grande superficie sur une couche de sol d'épaisseur limitée. Les mouvements du sol sont essentiellement des tassements verticaux, et seuls les bords du remblai ou du radier chargent le sol de manière différente, à savoir déviatorique : c'est ce dernier aspect qui limite les charges supportables et dicte la méthodologie de construction pour les remblais.

L'essai œdométrique est surtout pratiqué sur des sols vraiment compressibles et saturés, mais rien n'empêche de le réaliser sur tout type de sol; c'est rarement le cas, du moins pour la pratique courante. Des essais de type 

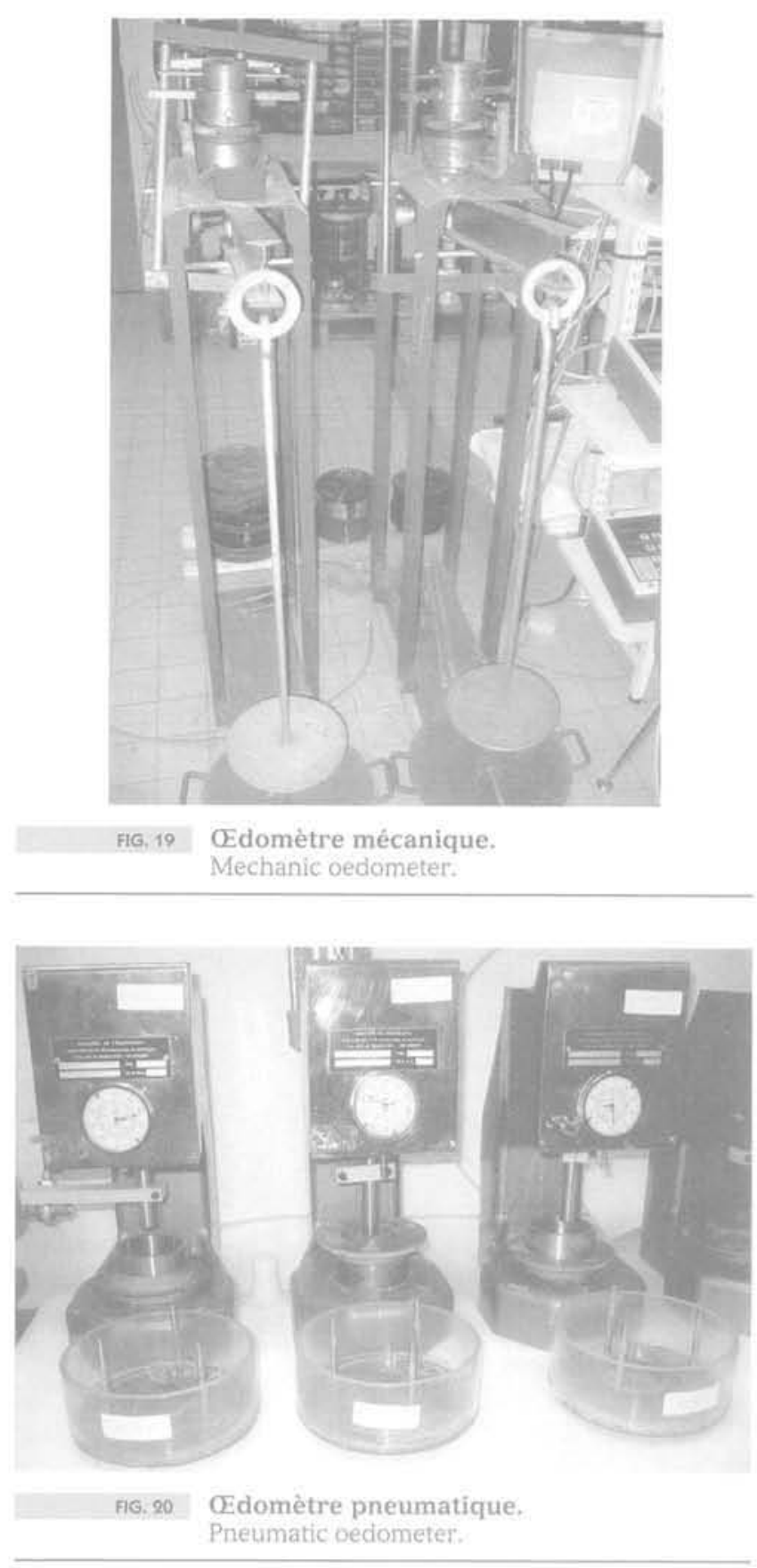

particulier ont été développés, tels ceux permettant la mesure du coefficient des terres au repos $K_{n}$.

$\mathrm{Si}$, comme dans l'essai triaxial, on pratique l'essai en empèchant tout drainage, on peut définir un module non drainé, qui ne présente aucun intérêt. comme le montre l'expression, applicable en élasticité, $E_{\mathrm{ced}, \mathrm{u}}=\frac{E_{\mathrm{u}}\left(1-v_{\mathrm{u}}\right)}{\left(1+v_{\mathrm{u}}\right)\left(1-2 v_{\mathrm{u}}\right)}$, dans laquelle, pour les argiles molles saturées, $v_{u}$ vaut 0,5 ou en est très proche. $\mathrm{E}_{\text {crdu }}$ est dans ce cas très élevé. Par contre, si l'on permet le drainage, et c'est tout l'intérêt de cet essai, destiné à l'étude de la consolidation (c'est-à-dire du report progressif des charges appliquées, de l'eau sur les grains, et de l'évolution du tassement avec le temps), on mesure et définit un module cedométrique $E_{m d^{\prime}}^{\prime} d^{\prime}$ où l'on peut tirer un module de type drainé $E^{\prime}$, par la relation $E_{\text {ced }}^{\prime}=\frac{E^{\prime}\left(1-v^{\prime}\right)}{\left(1+v^{\prime}\right)\left(1-2 v^{\prime}\right)}$.

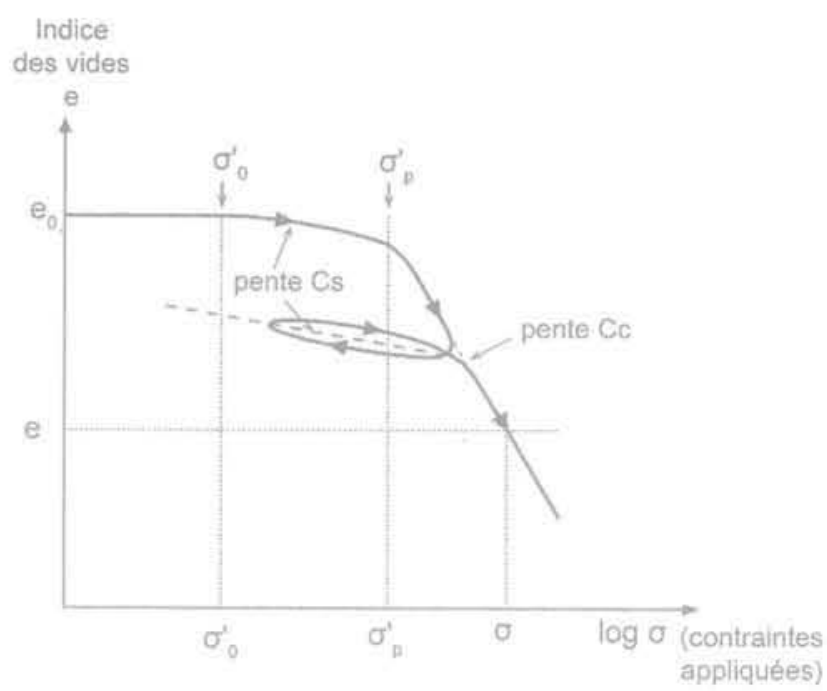

FIG. 21 Courbe de tassement cedométrique. Oedometric settlement curve.

Par définition le module odométrique sécant $E_{\text {ced }}^{\prime}=\frac{\sigma_{z}}{\varepsilon_{2}}$, où $\sigma_{z}$ est la contrainte appliquée lors de l'essai et $\varepsilon_{2}$, le tassement relatif final de Yéchantillon, en fin de consolidation.

D'un point de vue pratique, l'essai est directement interprété à partir de la courbe dessinée figure 21 et donnant l'indice des vides e, en fonction du logarithme décimal de la contrainte appliquée. On a la chance que cette courbe puisse être assimilée à un segment de droite pour une large plage de contraintes et on peut écrire $\varepsilon_{2}=\frac{\Delta L}{L}=\frac{C c}{1+e_{0}} \log \frac{\sigma_{z}+\sigma_{0}^{\prime}}{\sigma_{0}^{\prime}}$, où $e_{0}$ est l'indice de vide initial, $\mathrm{Cc}$ est le coefficient de compressibilité et $\sigma_{0}^{\prime}$ est la contrainte effective verticale régnant dans le massif au niveau du prélèvement de sol ; cette expression concerne les sols dits normalement consolidés qui n'ont jamais subi de contraintes verticales supérieures à celles existant lors du prélèvement.

Le module œdométrique, tel que défini ci-dessus sous forme d'un module sécant, est donc variable avec la contrainte appliquée, ce qu'il ne faut jamais oublier. Si $\sigma_{z}$ est très petit devant $\sigma_{0}, E_{\text {oed }}^{\prime} i=\frac{\sigma_{0}^{\prime}\left(1+e_{0}\right)}{0,435 \mathrm{Cc}}$ définit un module oedométrique initial, également variable puisqu'il augmente linéairement avec la profondeur $\left(\sigma_{0}^{\prime}=\gamma^{\prime} z\right)$ si e est considéré constant. En réalité E légèrement moins vite que $z$. On peut définir dè même, le module œdométrique tangent en tout point de la courbe $\left(\varepsilon_{z}, \sigma_{z}\right)$, qui s'exprime par $\frac{\sigma_{0}^{\prime}\left(1+e_{0}\right)}{0,435 \mathrm{CC}}\left(\sigma_{0}^{\prime}+\sigma_{z}\right)$ très explicite quant à sa variation avec $\sigma_{0}^{\prime}$ et $\sigma_{\gamma}$.

Deux exemples numériques permettent d'apprécier l'amplitude des variations du module, choisi ici sécant.

1) Une arqile molle très compressible saturée (caractérisée par $\mathrm{w}_{\text {cat }}=1 ; \mathrm{Cc}=0,8 ; \mathrm{e}_{0}=2,6$; et $\gamma_{\mathrm{h}}=14,5 \mathrm{kN} / \mathrm{m}^{3}$ ) est chargée sous $20 \mathrm{kPa}$, puis $140 \mathrm{kPa}$ (remblai de $1 \mathrm{~m}$, puis $7 \mathrm{~m}$ ). Les valeurs de $\mathrm{E}^{\prime}$ à $5 \mathrm{~m}$ puis $10 \mathrm{~m}$ de profondeur sont données en $\mathrm{kPa}$ dans le tableau I ci-dessous, indiquant également la valeur du module initial sous charge nulle; y figure également la valeur du tassement d'une épaisseur de $1,00 \mathrm{~m}$ de sol, à la profondeur considérée. 
TABLEAU I Valeurs des modules œedométriques (en kPa) et tassements dans une argile molle.

Values of oedometric moduli and settlements in soft clay.

\begin{tabular}{|c|c|c|}
\hline Charge Profondeur 2 & $5 \mathrm{~m}$ & $10 \mathrm{~m}$ \\
\hline 0 & 230 & 460 \\
\hline $1 \mathrm{~m}(20 \mathrm{kPa})$ & $3206 \mathrm{~cm}$ & ${ }^{560} 3 \mathrm{~cm}$ \\
\hline $7 \mathrm{~m}(140 \mathrm{kPa})$ & $19 \mathrm{~cm}$ & $\begin{array}{l}1020 \\
14 \mathrm{~cm}\end{array}$ \\
\hline
\end{tabular}

2) Un limon légèrement compressible, non saturé $\left(\mathrm{w}=0,2 ; \mathrm{Cc}=0,1 ; \mathrm{e}_{0}=0,6 ; \gamma_{\mathrm{b}}=18,5 \mathrm{kN} / \mathrm{m}^{3}\right)$, donne dans les mêmes configurations, les valeurs indiquées au tableau II.

TABLEAUII Valeurs des modules œedométriques (en $\mathrm{kPa}$ ) et tassements dans un limon. Values of pedometric moduli and settlements in lime.

\begin{tabular}{lcc}
\hline Charge & $5 \mathrm{~m}$ & $10 \mathrm{~m}$ \\
\hline 0 & & \\
\hline $1 \mathrm{~m}(20(\mathrm{kPa})$ & 3400 & 6800 \\
\hline $7 \mathrm{~m}(140 \mathrm{kPa})$ & 3800 & 6900 \\
& $0.5 \mathrm{~cm}$ & $0.3 \mathrm{~cm}$ \\
\hline & 5600 & 9140 \\
& $2.5 \mathrm{~cm}$ & $1.5 \mathrm{~cm}$ \\
\hline
\end{tabular}

Ces valeurs et leurs variations montrent évidemment que si le sol est peu compressible, admettre, pour une profondeur $z$ donnée, la constance du module œdométrique avec la charge appliquée reste sans grande incidence. Ainsi dans l'exemple 2, pour les couches situées vers 10 mètres, on peut choisir une valeur unique $E_{\text {mid }}^{\prime}=8000 \mathrm{kPa}$, quelle que soit la charge de remblai ; les tassements calculés sont en effet très faibles et, de ce fait, a priori peu préoccupants pour le projet. L'argile molle par contre ne peut, sans grosse erreur relative sur les tassements qui sont importants, faire l'objet de la même simplification.

Mais considérer une valeur unique du module sur toute l'épaisseur du massif de sol est faux et ne peut être acceptable que pour des épaisseurs modestes.

On met de la même manière en évidence l'effet d'un déchargement-rechargement qui conduit à un coefficient de gonflement Cs très inférieur à Cc. La courbe de l'essai œdométrique reflète donc tout logiquement une allure analogue à celle d'un essai triaxial, avec cycle.

Les massifs de sol ayant subi autrefois une contrainte statique $\sigma^{\prime}$ importante aujourd'hui disparue sont dits surconsolidés. On conçoit que le tassement entraîné par leur rechargement soit sans grande incidence si ce dernier n'entraîne qu'une contrainte inférieure à $\sigma_{p}^{\prime}$. Par contre, si elle dépasse $\sigma_{p^{\prime}}^{\prime}$ la fraction supérieure à $\sigma_{p}^{\prime}$ conduit à un tassement dont l'amplitude est régie par le coefficient $\mathrm{Cc}$.
On a dans ces deux situations, où $\sigma^{\prime}$ représente la contrainte qui a régné au niveau considéré, les relations suivantes :

$$
\begin{array}{ll}
\varepsilon_{2}=\frac{\operatorname{Cs}}{1+\varepsilon_{0}} \log \frac{\sigma_{z}+\sigma_{0}^{\prime}}{\sigma_{0}^{\prime}} & \text { si } \sigma_{z}+\sigma_{0}^{\prime}<\sigma_{p}^{\prime} \\
\varepsilon_{2}=\frac{1}{1+e_{0}}\left(\operatorname{Cslog} \frac{\sigma_{p}^{\prime}}{\sigma_{0}^{\prime}}+\operatorname{Cclog} \frac{\sigma_{z}+\sigma^{\prime}}{\sigma_{p}^{\prime}}\right) & \text { si } \sigma_{z}+\sigma_{0}^{\prime}<\sigma_{p}^{\prime} .
\end{array}
$$

Si l'on reprend le premier exemple précédent d'argile molle, en introduisant une surconsolidation $\sigma_{p}^{\prime}=\sigma_{0}^{\prime}+30 \mathrm{kPa}$ et $\mathrm{Cs}=0,15$, les valeurs des tassements et des modules équivalents sont fortement modifiés, comme l'indique le tableau III ci-dessous, et ce, dans des proportions très variables suivant la charge appliquée.

TABLEAU III Valeurs des modules cedométriques (en kPa) et tassements dans une argile molle surconsolidée.

Values of oedometric moduly and settlements in an overconsolidated soft clay.

\begin{tabular}{lcc}
\hline Profondeur $z$ & $5 \mathrm{~m}$ & $10 \mathrm{~m}$ \\
\hline $1 \mathrm{~m}(20 \mathrm{kPa})$ & & \\
& 1740 & 3000 \\
& $1,1 \mathrm{~cm}$ & $0,7 \mathrm{~cm}$ \\
\hline $\mathrm{m}(140 \mathrm{kPa})$ & 1125 & 1460 \\
& $12,4 \mathrm{~cm}$ & $9,6 \mathrm{~cm}$ \\
\hline
\end{tabular}

Il faut surtout remarquer dans ce tableau, la chute du module, et donc l'augmentation forte du tassement, dès que la charge appliquée $\sigma_{2}$ a dépassé la valeur de $30 \mathrm{kPa}$.

Aussi la détermination de $\sigma^{\prime}$, est elle très importante pour certains types d'ouvrages, alors que l'incidence l'est beaucoup moins pour d'autres. Si l'argile molle de ce premier exemple ne consiste qu'en une couche unique de $1,00 \mathrm{~m}$ d'épaisseur située à $5,00 \mathrm{~m}$ de profondeur moyenne (les sols sous et sus jacents étant supposés quasiment incompressibles), le fait d'ignorer la surconsolidation, conduit pour un remblai de $7,00 \mathrm{~m}$ $(140 \mathrm{kPa})$ à prévoir un tassement de $19 \mathrm{~cm}$ au lieu de $12,4 \mathrm{~cm}$, ce qui n'est pas très grave en soi compte tenu de la nature de l'ouvrage. Par contre, s'il s'agit de mettre en place un dallage ou un radier, ouvrages sensibles, apportant $20 \mathrm{kPa}$ de charge, les répercussions ne sont pas du tout les mêmes, puisque pour $1,1 \mathrm{~cm}$ a correctement s prévisible, on annoncera $6 \mathrm{~cm}$. Il sera alors sans doute préconisé des travaux particuliers, tels une surcharge provisoire (ce qui se fait actuellement assez peu) ou plus souvent une amélioration des sols, qui n'a pas obligatoirement lieu d'être.

Ce cas de figure des dallages industriels est courant, et pour des faciès da sols qui souvent ne sont que légèrement compressibles, souvent légèrement surconsolidés et assez peu chargés. Une préoccupation liée étroitement à ce type de problème est celle de la connaissance des niveaux de la nappe phréatique, si elle existe et, tout particulièrement, de son niveau le plus bas, lequel concourt à la surconsolidation.

Il faut reconnaitre que la détermination de $\sigma^{\prime}$ est difficile et demande en particulier un très grand soin lors du prélèvement et de la préparation des échan- 
tillons de sols. L'utilisation du piézocône, avec une mesure en places s'avère tout à fait intéressante.

L'essai cedométrique permet également d'aborder l'étude du fluage particulièrement pour les sols dont la structure est sujette à ce phénomène : les vases argileuses, les tourbes. Ce phénomène, qui se traduit physiquement par des déformations différées à long terme, dénommé également compression secondaire, se superpose et surtout prolonge celui qui vient d'être examiné, lié à la consolidation primaire. Ces déformations différées s'ajoutent aux déformations précédentes ; l'essai de fluage, adapté à cet effet permet d'en prévoir l'amplitude en fonction du temps.

Il faut donc retenir que le module codométrique sous sa forme sécante, caractérise la transformation d'une couche de sol situé à un niveau 2 , sous une contrainte imposée. Ce module est essentiellement variable en fonction de la profondeur. Après la consolidation sous cette contrainte, le sol final est mécaniquement différent du sol initial : il est un peu plus dense et ses caractéristiques sont meilleures. C'est ce que recherchent les méthodes d'amélioration des sols, où l'on met en ouvre des techniques diverses : préchargement avec ou sans drainage, pilonnage, consolidation par le vide... précisément destinées à l'obtention d'un sol, après traitement, beaucoup moins déformable qu'initialement.

\section{4}

\section{Relations entre essais triaxiaux, œdométriques et autres essais}

Dans un essai œdométrique, les modules $E_{\text {'d }}^{\prime}=\frac{\sigma_{z}}{\varepsilon_{z}}$ et $E^{\prime}$ qu'on peut en déduire sont, du moins pour les sols normalement consolidés, croissants avec la contrainte appliquée, alors que le module de même nature drainé, $E^{\prime}$, que l'on tire de l'essai triaxial, est décroissant avec le déviateur appliqué. Les deux valeurs de $E^{\prime}$ obtenues par l'un ou l'autre essai ne sont donc a priori pas trop différentes que pour des contraintes de cisaillement ou de compression extrêmement faibles (modules initiaux), et pour des échantillons de sols qui sont dans des états initiaux de contraintes strictement identiques.

Ces deux essais révèlent une différence importante entre le comportement réel du sol et un comportement élastique. Elle est très fortement accentuée par la coexistence de deux états, déviatorique et sphérique, qui s'opposent et qui contribuent aux déformations au sein du massif par deux manifestations distinctes: déplacement par glissement des grains entre eux ou compression.

L'essai triaxial, suivant ses modalités, permet l'accès à quatre paramètres de déformabilité $E_{u,} v_{u}$ et $E^{\prime}, v^{\prime} ; E_{u}$ et $E^{\prime}$ sont variables avec la contrainte appliquée. On a indiqué que $E^{\prime} \leq E_{\text {, }}$, et les valeurs de ces modules sont d'autant plus élevées que le sol est dense. Une légitime interrogation concerne l'éventuelle relation entre ces deux grandeurs. Il a été montré (Giroud, 1975), dans l'essai de cisaillement simple, sans variation de volume, si le sol est parfaitement élastique, tant en conditions non drainées que drainées, que $\frac{1+v^{\prime}}{E^{\prime}}=\frac{1+v_{u}}{E_{u}}$. Ceci conduit, pour un sol pulvérulent où $v^{\prime}=v_{u^{\prime}}$ à $E^{\prime}=E_{u}$ et pour un sol fin saturé où $v_{u}=0,5$ à $E_{u}=\frac{1,5 E^{\prime}}{\left(1+v^{\prime}\right)}$. Cette

égalité n'est cependant strictement vraie pour le sol que dans le domaine des petites déformations, où l'on peut légitimement admettre que le sol considéré dans l'une ou l'autre des deux conditions reste strictement le même, le départ d'eau par drainage étant totalement négligeable. Ce n'est pas le cas pour les ouvrages géotechniques.

Ce rapport théorique $\frac{E_{u}}{E^{\prime}}$ pour les sols fins, est très faible si on le compare à la réalité mesurée, située le plus souvent entre 1 et 10. Les explications de cette très grande différence sont à rechercher au niveau du comportement de chacune des phases constituant le sol : compressibilité du squelette, des gaz (pour les sols non saturés), dissolution des gaz en fonction des pressions appliquées et quasi incompressibilité de l'eau.

On retiendra sous certaines hypothèses restrictives nécessitant un comportement élastique des éléments constitutifs du sol, loin de la rupture du sol, et dans un domaine limité de contraintes, les relations établies pour l'essai triaxial et dont on trouvera le principe de leur obtention par exemple chez Giroud (Giroud, 1975):

$$
E_{u}=\frac{E^{\prime}}{1-A B\left(1-2 v^{\prime}\right)} \text { ou } E^{\prime}=\frac{E_{u}(1-B)}{1-B+A B(1-2 v u)}
$$

Les mécaniciens des sols reconnaîtront ici les coefficients A et B de Skempton qui permettent de relier la surpression interstitielle $\Delta \mathrm{u}$ aux accroissements $\Delta \sigma_{1}$ et $\Delta \sigma_{3}$ des contraintes principales, par la relation $\Delta \mathrm{u}=\mathrm{B}\left[\Delta \sigma_{3}+\mathrm{A}\left(\Delta \sigma_{1}-\Delta \sigma_{3}\right)\right]$.

D'un point de vue pratique, lorsque le degré de saturation en eau du sol est inférieur à 0,7 , alors $B \cong 0$ et $E_{u}=E^{\prime}$; si le sol est saturé $B=1$ et $v_{u}=0,5$; les ordres de grandeur de $\mathrm{A}$ et des rapports qui en découlent sont fournis dans les tableaux IV.a et IV.b ci-après.

En conclusion de l'examen des essais de laboratoire de base que sont l'essai triaxial avec ses modalités différentes et l'essai œdométrique, on retiendra que les caractéristiques de déformabilité $E_{*}, E^{\prime}$ ou $E_{\text {ond }}$ sont variables avec les contraintes appliquées et avec le type de sollicitation appliquée. Mais, dans les domaines de

TABLEAU IV.a Ordres de grandeur du paramètre A (relatif à de petites déformations) en fonction du degré de consolidation de I'argile (d'après Skempton et Bjerrum). Approximation of Skempton coefficient A with the degree of consolidation of clay.

\begin{tabular}{lr}
\hline Argile très surconsolidée & $0 \leq \mathrm{A} \leq 0,25$ \\
Argile surconsolidée & $0,25 \leq \mathrm{A} \leq 0,5$ \\
Argile normalement consolidée & $0,5 \leq \mathrm{A} \leq 1$ \\
Argile molle et sensible & $\mathrm{A} \geq 1$ \\
\hline
\end{tabular}

TABLEAUIV.b Ordres de grandeur du rapport des modules non drainé et drainé, en sols fins saturés.

Approximation of undrained and drained moduli ratio in saturated fine solis.

Argile très surconsolidée

Argile surconsolidẻe $1 \leq \frac{E_{u}}{E^{\prime}} \leq 1,2$

Argile normalement consolidée $1,1 \leq \frac{E_{u}}{E^{\prime}} \leq 1,5$

Argile molle et sensible $1,2 \leq \frac{E_{u}}{E^{\prime}} \leq 2,5$ $1,5 \leq \frac{E_{u}}{E^{\prime}} \leq 10$ 
déformations très limitées, ces grandeurs peuvent être reliées ou corrélées entre elles pourvu qu'elles le soient dans des plages de contraintes qui soient proches, ce qui atténue l'incidence de la non élasticité, ainsi que dans des états qui soient comparables. Ces conditions limitent fortement les possibilités de passer d'une caractéristique à l'autre, les difficultés croissant avec les sols de plus en plus mous.

Certains ouvrages géotechniques conduisent par contre à de très fortes déformations. Le cas du remblai sur sol compressible épais en est l'exemple où, après tassement et donc après drainage, le sol compressible est totalement différent de ce qu'il était initialement.

La comparaison sans précautions, dans de telles conditions, d'un module non drainé avec un module drainé peut percire toute signification et le rapport, tel que défini à partir des tableaux IV, peut être totalement bouleversé et sans signification. La figure 29 relative aux fondations superficielles en est d'ailleurs la parfaite Illustration ; à la charge $Q$ appliquée correspond deux valeurs de tassement à court et long terme. Le rapport des modules sécants correspondants E/E' varie : supérieur à 1 aux faibles charges, atteignant 1 , puis pouvant atteindre de très faibles valeurs pour des charges importantes. La mobilisation des caractéristiques de cisaillement non drainées et drainées altère profondément les relations liant les modules.

Les exemples numériques menés à partir de résultats cedométriques, et les tableaux IV sont très instructifs, puisqu'ils montrent, en particulier pour des argiles surconsolidées et très surconsolidées, des rapports $\frac{E_{u}}{E^{\prime}}$ assez proches de 1 ; on peut donc imaginer pour ces dernières d'accéder à un comportement drainé, permettant de prévoir un tassement ou une déformation à long terme, à partir d'un essai non drainé, rapide.

Pour les argiles normalement consolidées et a fortiori molles et sensibles, cette démarche est évidemment déconseillée.

Un dernier point relatif à la détermination des modules concerne les incertitudes qui affectent les valeurs auxquelles on aboutit. Elles résultent des mesures elles-mèmes, mais l'essentiel provient du

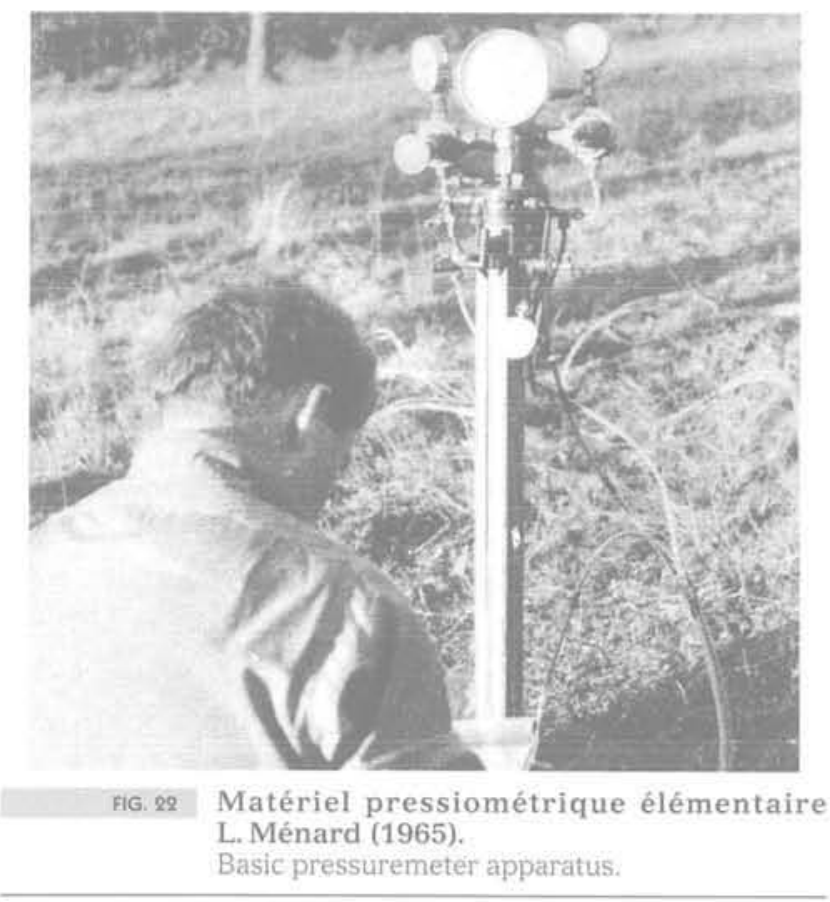

conditionnement de l'éprouvette qui inclut le forage, le prélèvement, le transport et la préparation en laboratoire, L'influence de cette succession de facteurs peut être très importante ; elle est en tout cas très variable suivant la nature et l'état des sols en place. La norme P-94202 sur les prélèvements a pour objet de permettre de limiter cette influence.

Si l'on étend aux autres types d'essais cette comparaison, il est essentiel de retenir que les modules déterminés par les techniques de propagation des ondes sont évidemment très largement supérieurs à ceux résultant de l'utilisation d'autres techniques. Ainsi, les valeurs suivantes extraites d'une étude sur la déformabilité d'un massif marneux (Magnan et al., 1998) le rappellent. Outre une croissance systématique avec la profondeur du module quel qu'en soit le type, on a mesuré les fourchettes suivantes de $E_{\max } / E$ où $E_{\max }$ est le module déterminé par ondes de surface et $\mathrm{E}$ celui issu d'un autre mode :

- avec les essais de laboratoire : 25 à 50 (œedomètres et triaxiaux);

- avec les essais pressiométriques Ménard: 15 à 30; - avec des mesures de tassement en profondeur par couches : 2 à 20 .

C'est la prise en compte de ces variations, liées à des niveaux très variables de sollicitations du sol ou du massif, qui est nécessaire pour construire des modèles réalistes de lois de comportement des massifs et permettre la résolution de problèmes délicats de déformation.

\section{5}

\section{L'essai pressiométrique}

Cet essai, réalisé en place sur le terrain, est apparu il y a cinquante ans dans la pratique de la mécanique des sols, avec le dépôt d’un brevet, le 9 janvier 1955, par Louis Ménard (1931-1978). Il est devenu, au moins en France, un essai de routine très largement utilisé maintenant.

Il permet une approche différente des problèmes géotechriques et la réalisation d'un nombre plus important d'essais que le laboratoire ne le permet.

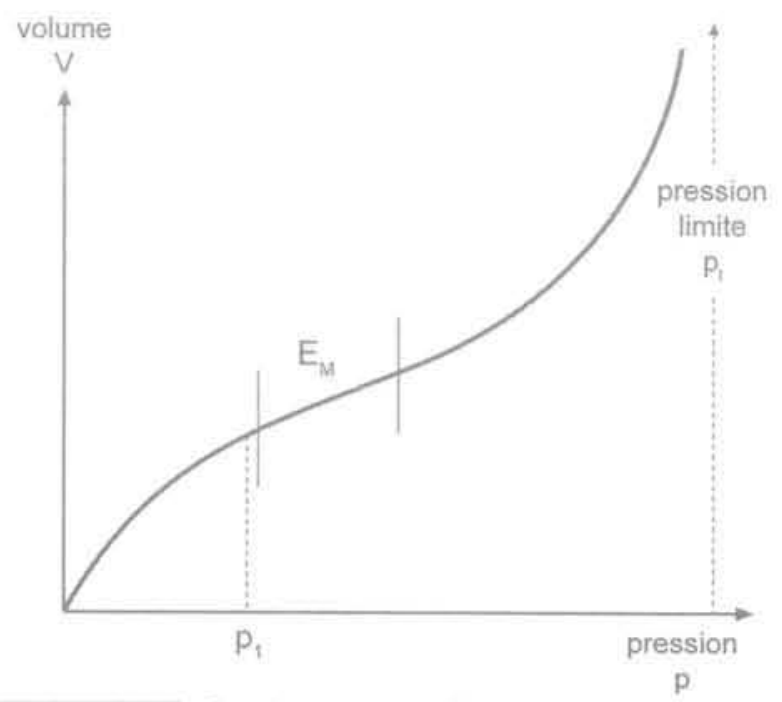

FIG, 23 Courbe pressiométrique type. Pressuremeter type curve. 
Le pressiomètre Ménard comprend essentiellement une sonde cylindrique introduite dans un forage réalisé à l'avance et de diamètre légèrement plus grand que la sonde. Celle-ci est dilatable radialement sous l'effet d'une pression fournie par un contrôleur pression volume (CPV) situé au niveau du sol. La sonde comprend trois cellules : une cellule centrale pleine d'eau et deux cellules de « garde » de manière à ce que contraintes et déformations induites par la cellule centrale dans le sol soient radiales. Elle est reliée au CPV par des tubulures souples. Le contrôleur donne l'augmentation de diamètre du forage par la mesure du volume de la cellule centrale. On réalise ainsi un essai statique de chargement en augmentant la pression par paliers de pression égaux. L'interprétation de la courbe de chargement permet d'en déduire :

- un module de déformation, en phase dite pseudoélastique, car elle est pratiquement linéaire, mais non réversible ;

- une pression limite qui correspond à l'apparition de grandes déformations de la cavité cylindrique.

Une courbe pressiométrique type est donnée en figure 23 ; elle présente trois parties :

- la première correspond à une remise en contact entre sonde et paroi du forage. On peut dire qu'on remédie en partie au remaniement du sol et à sa décompression; on atteint de la sorte une pression $\mathrm{p}_{1}$;

- la deuxième n'est pas linéaire mais s'en rapproche : elle permet de ce fait le calcul d'un module dit pressiométrique, noté $\mathrm{E}_{\mathrm{M}}$, défini sur une plage de contrainte dont la borne supérieure ne dépasse pas une valeur dite pression critique (ou de fluage) valeur au-delà de laquelle la troisième partie de la courbe présente rapidement une allure asymptotique qui conduit à définir la pression limite $\mathrm{p}_{\mathrm{p}}$.

Le module $G=\frac{E}{2(1+v)}$, on l'a vu en partie 1, est défini à partir de la relation $G=V_{0} \frac{p-p_{0}}{V-V_{0}}$ découlant, en assimilant le sol à un matériau élastique (qu'il n'est pas), de l'expression exacte $e V=V_{0} e^{\frac{p-P_{0}}{G}}$ sachant que $\frac{p-P_{0}}{G}$
est souvent petit.

De cette valeur de G, avec l'hypothèse faite d'un coefficient de Poisson de 0,33, la valeur de $\mathrm{E}_{\mathrm{M}}$ qu'on en déduit est utilisée pour le calcul du tassement des fondations, voire des remblais ou radiers sur sols compressibles ; nous y reviendrons.

Il est utile de rappeler que le module $\mathrm{E}_{\mathrm{M}}$ était, dans les années soixante, obtenu à partir d'essais effectués dans des forages réalisés en tarière à main ; il s'agissait de formations où une telle pratique est possible, en l'occurrence les sols fins hors nappe. Le soin pris à l'exécution de ces avant-trous était tout à fait remarquable. Le module obtenu était considéré comme le module de référence et les essais de chargement expérimentaux de semelles nécessaires au calage des méthodes de calcul de tassement à partir de $\mathrm{E}_{\mathrm{M}^{\prime}}$ ont été basés sur ces valeurs de référence.

Le souci d'une plus grande rapidité d'exécution des essais et d'une réalisation dans des terrains autres que fins, a conduit à multiplier les types de matériels de forages. Aussi a-t-on été conduit à étudier, pour différents types de sols, l'influence du mode de forage sur les valeurs de $\mathrm{E}_{\mathrm{M}}$ et de la pression limite $\mathrm{p}_{\mathrm{l}}$. Des recom-

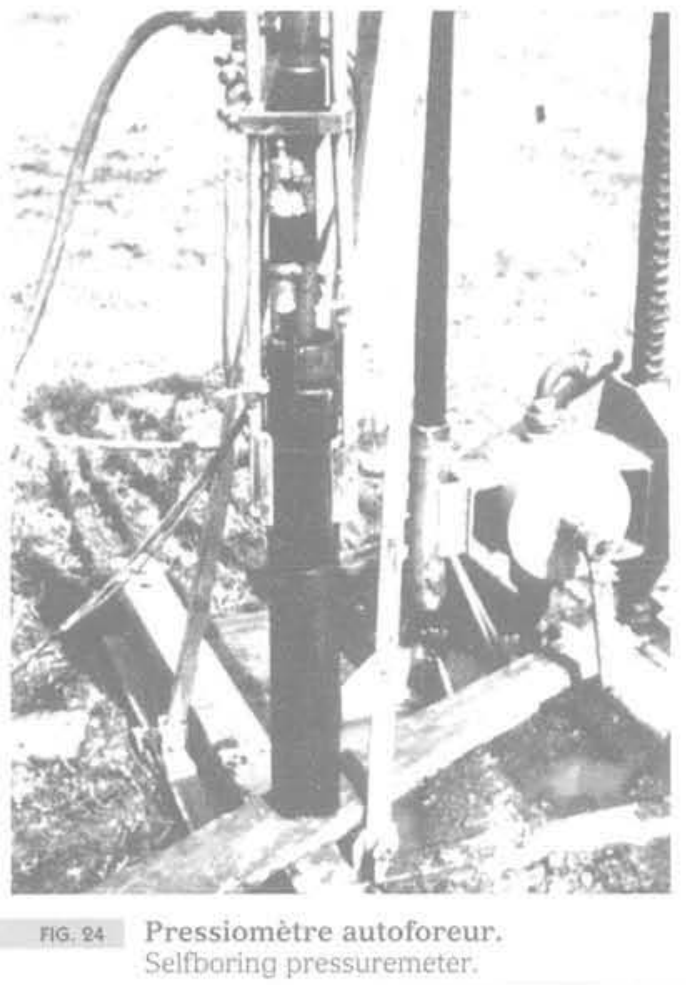

mandations ont donc été introduites, d'abord dans le mode opératoire initial (LCPC, 1971) puis dans la norme actuelle sur l'essai pressiométrique (Afnor, 2000) qui indiquent les méthodes les plus appropriées à utiliser. C'est sous cette condition que l'utilisation qui est faite du module $E_{M}$ pour le calcul des déformations est appropriée. Malgré cela, on constate des écarts dans la mise en œuvre, qui ont une forte influence sur les valeurs des modules calculés; les répercussions sur les tassements prévisibles sont évidentes.

Même réalisé avec le soin exigê par les normes, le forage perturbe la réponse du sol; le sol est donc remanié, mais on peut considérer que ce remaniement est en quelque sorte contrôlé et encadré. Le massif n'est efficacement sollicité qu'à partir du point d'inflexion apparaissant sur la courbe volume-pression. C'est d'ailleurs précisément autour de ce point que l'on peut définir le « meilleur» module. Néanmoins, certains sols subissent des modifications, telles les arènes granitiques pouvant passer de la « microroche » au sol fin; l'effet sur la valeur du module du massif reste sans doute limité. A des fins plus fondamentales, le pressiomètre a été l'objet, à partir de 1970 (Baguelin et al., 1978), de recherches technologiques puis théoriques, très importantes qui ont conduit à la mise en œuvre par « autoforage ».

Pour simplifier, la méthode consiste à mettre en œuvre dans le sol la sonde pressiométrique sans qu'il y ait modification de l'état initial du sol. L'essai pressiométrique d'expansion, alors réalisé, permet de charger un sol vierge dont on peut analyser les propriétés élémentaires.

Toute la difficulté dans l'autoforage a été de permettre la réalisation d'un forage sans qu'il y ait perturbation du sol, ou du moins qu'elle soit la plus réduite possible; ce sont plusieurs années d'efforts technologiques dès les années 70, sous l'impulsion de F. Jézequel, du laboratoire des Ponts et Chaussées de SaintBrieuc, qui ont permis d'aboutir et de permettre le développement d'essais en place de grande qualité, dans les sols fins. 
Ces essais peuvent être qualifiés, comme on l'a fait pour le laboratoire, d'essais élémentaires; l'essai pressiométrique "autoforé » en est un. Son utilisation en tant que tel n'a cependant pas connu de grands développements du fait d'une orientation du pressiomètre, à caractère très opérationnel, à savoir le dimensionnement des fondations. Le non-succès de cette voie, du fait d'une haute technicité nécessaire et de méthodes existantes suffisantes a conduit, du moins en France, à une longue période de délaissement. Actuellement, l'autoforage revoit le jour, avec l'optique de la caractérisation élémentaire du sol qui aurait toujours dû être la sienne et pour laquelle les outils et justifications existent depuis I'origine du développement.

La figure 25 illustre simultanément une courbe issue de l'essai standard (3) et des courbes vierges issues d'essais " autoforés ») (1) pour un sol de type élastoplastique (Mohr - Coulomb) et (2) pour un sol réel.

La courbe (2) présente qualitativement une allure similaire à celle donnée par un essai triaxial; le module

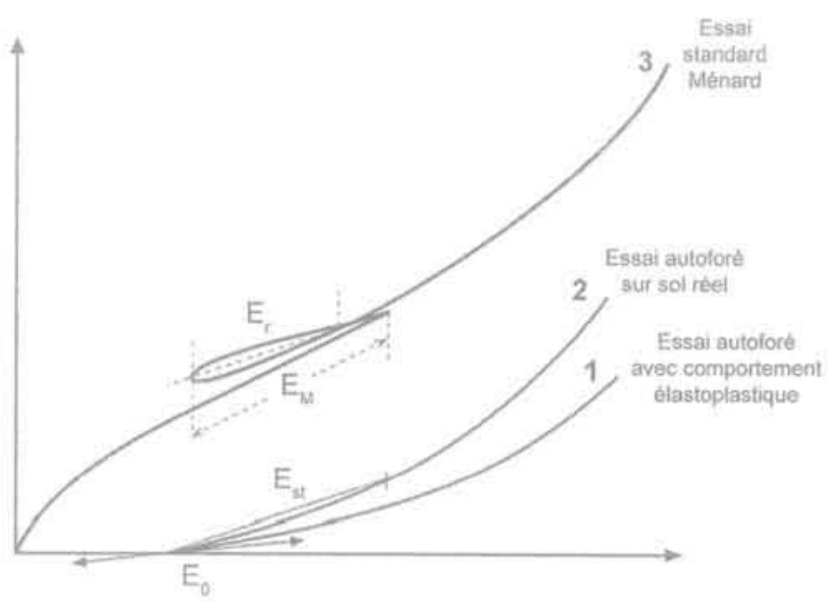

FIG. 25 Courbes pressiométriques standard et par autoforage.

Pressumeter curves : standard and pressuremeters.

$E_{0}$ initial tangent, et les modules successifs sont très largement supérieurs aux modules définis sur la courbe standard (3) pour des plages de contraintes identiques.

La réalisation d'un cycle de déchargement-rechargement conduit à un module cyclique $\mathrm{E}$ qui se rapproche de la valeur du module sécant $\mathrm{E}_{\text {, }}$ de la courbe 2, pour une plage de contraintes identique. Ainsi, si un ouvrage sollicite le sol pour cette plage de contraintes, et mobilise donc $E_{s}$ l'utilisation de $E_{r}$ s'avère-t-elle justifiée.

Aussi l'idée toute naturelle d'introduire dans l'essai standard un cycle de déchargement-rechargement dans la plage de contraintes utilisée pour le calcul de $E_{M}$ a-t-elle surgi très tôt, chez L. Ménard lui-même (Ménard, 1962) dans le but d'atteindre un module élastique appelé à l'époque module de microdéformation. Les essais réalisés, limités en nombre, ont donné des résultats néanmoins intéressants.

Ce type de démarche a été plus récemment repris dans le réseau des laboratoires des Ponts et Chaussées. et une synthèse des résultats a été publiée (Combarieu, Canepa, 2001) portant sur l'influence du mode de forage et l'obtention de rapports caractéristiques $\frac{\text { Err }}{\mathrm{EM}}$ selon les natures des sols. Le module $\mathrm{E}_{\text {f }}$ est celui de rechargement assimilé à un module élastique moyen, calculé pratiquement dans la plage des contraintes choisie pour calculer $\mathrm{E}_{\mathrm{M}}$. Ce module présente de ce fait un intérêt pour aborder des calculs de tassements.

Le tableau V, extrait de cette synthèse, fournit ces rapports pour quatre natures de sols testés, représentant sept sites naturels étudiés et dans les conditions opératoires les meilleures.

TABLEAUV Essais pressiométriques cycliques rapports caractéristiques $\mathrm{E}_{/} / \mathrm{E}_{\mathrm{Y}}$ Cyclic test with pressumeter ; usual ratio $E_{1} / E_{M}$.

\begin{tabular}{lc} 
Sol ètudié & $\mathrm{E}_{\mathrm{A}} / \mathrm{E}_{\mathrm{dx}}$ \\
\hline Argile raide surconsolidée & 2,5 a 3,5 \\
Limon & 3 a 4,5 \\
Sable & 6 à 7,5 \\
Craie & 5,5 \\
\hline
\end{tabular}

Ces valeurs rejoignent celles données pour les trois premiers sols étudiés par L. Ménard. Toutefois, dans l'argile raide par exemple, on a mesuré $E_{/} / E_{M}=1$, avec une mise en cuvre, tout à fait proscrite, de la sonde de mesure par battage direct.

Une première remarque capitale s'impose : l'essai pressiométrique est un essai qui sollicite rapidement le sol ; les paliers de chargement sont d'ailleurs appliqués une minute ; c'est un essai non drainé pour les sols fins, et il est donc vain de vouloir approcher des phénomènes de consolidation, à long terme, avec cet essai.

Une seconde remarque a trait à la signification mécanique de $\mathrm{E}_{\mathrm{M}}$ ou $\mathrm{E}_{\mathrm{r}}$. Ce sont certes des modules, mais qui caractérisent le comportement horizontal du massif du sol, alors que ceux mesurés lors d'essais triaxiaux ou œdométriques intéressent en majorité le comportement vertical. Il s'agit de l'anisotropie pour laquelle il sera donné plus loin quelques éléments.

Il faut donc retenir de l'essai pressiométrique, que le module $\mathrm{E}_{M}$ déterminé lors d'une campagne d'essais doit l'être avec le souci, pour celui qui paye les essais, du res-

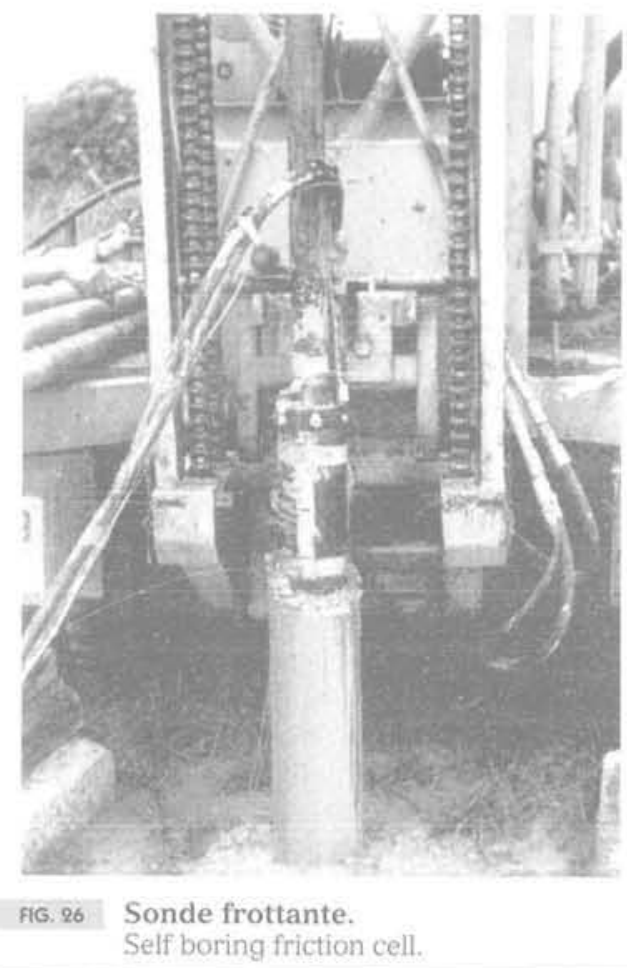


pect strict de la norme. De toute manière, il y a lieu de fournir les différents éléments permettant de juger de la qualité des résultats. Ainsi, la description de la méthode de forage devrait être systématiquement mentionnée, de même que la longueur de forage pratiquée avant l'introduction de la sonde. Les valeurs du coefficient $\alpha$, évoqué ci-après, ne sont en effet pas indépendantes de cette qualité et ont été fixées en partie dans l'hypothèse de la réalisation correcte des essais.

Un exemple volontairement extrême est celui d'un prestataire d'essai qui, dans le but d'une réalisation irréprochable d'essais, effectuerait ceux-ci par autoforage sans le spécifier. La fondation calculée par la méthode pressiométrique connaitrait sans doute quelques déboires.

De manière plus réaliste et courante, nous avons eu à examiner, pour le compte d'un maitre d'ouvrage, une note de calcul des fondations mettant en jeu sur 4,00 m d'épaisseur environ une couche de limon et d'argile peu compacte, proche de la surface et partiellement saturée. Le jeu d'appels d'offres successifs a conduit deux prestataires différents à intervenir.

La déformabilité du massif a conduit pour l'un à préconiser un module moyen $\mathrm{E}_{\mathrm{M}}$ de $1.7 \mathrm{MPa}$, et pour l'autre $3,4 \mathrm{MPa}$, sans qu'on puisse disposer de renseignements fiables pour juger de la plus ou moins bonne pertinence de ces résultats. La pression limite avait été heureusement, quant à elle, trouvée pratiquement identique d'une campagne à l'autre. D'autres exemples concrets ont en effet conduit à des rapports de 2 sur les pressions limites mesurées.

\section{6}

\section{Les essais de cisaillement en place}

L'autoforage a permis de réaliser in situ des essais qui peuvent être considérés comme des essais élémentaires. Ainsi l'essai à la sonde frottante, module de mesure qui succède au module d'autoforage, permet de construire à un niveau donné la courbe enfoncement - frottement unitaire de la sonde. Ce frottement caractérise le contact sol intact/acier, lors du chargement vertical imposé à la sonde. Ce type d'essai, qui consiste en un cisaillement pur a été étudié (et le développement théorique en élasticité linéaire isotrope a été mené conjointement) pour comprendre les mécanismes élémentaires de frottement le long des pieux.

Le frottement latéral f est donné par l'expression simple $\mathrm{f}=\mathrm{f}=\frac{2 \mathrm{G} \mathrm{y}_{0}}{\mathrm{kB}}$ où $\mathrm{G}$ est le module de cisaillement du sol, initial, sécant ou proche de la rupture, $\mathrm{y}_{0}$ est l'enfoncement de la sonde frottante de diamètre B et $k$ un coefficient essentiellement géométrique.

Cet essai n'a été pratiqué qu'à des fins de recherche et un des résultats importants a été de montrer, comprendre et valider la suffisance de très faibles déformations relatives sol-pieu, de l'ordre de 1/100 du diamètre de ce dernier, pour mobiliser le frottement ultime au contact.

L'essai à la sonde frottante, associé à des mesures de propagation d'ondes en place et à l'essai pressiométrique, permet d'accéder directement à la détermination du module de cisaillement. Ces outils peuvent donc concourir à construire la courbe, telle que la figure 27 le montre, donnant $\mathrm{G} / \mathrm{G}_{\max }$ en fonction de la déformation relative ou de la distorsion.

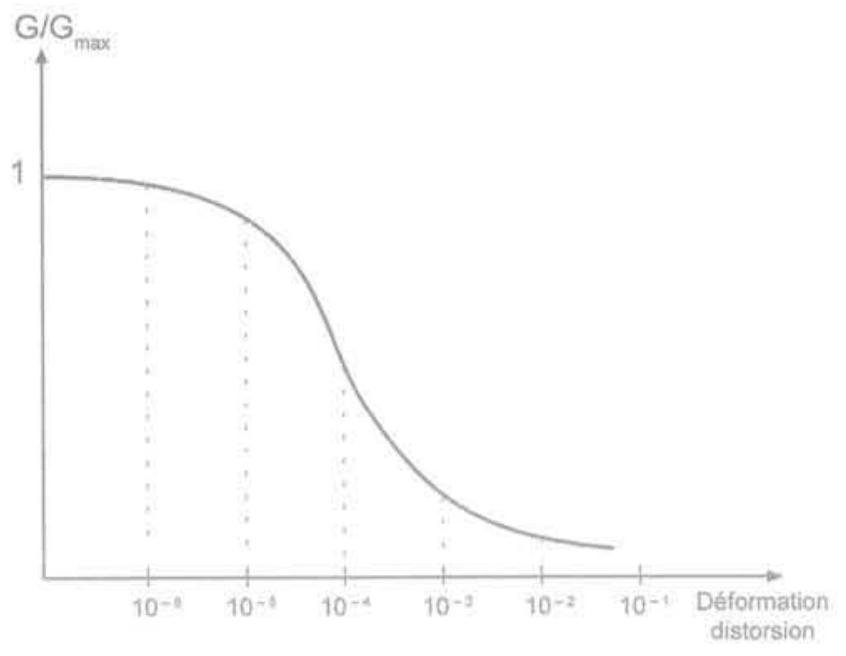

FIG. 27 Evolution du module $\mathrm{G}$ avec la déformation. Evolution of $G$ modulus with deformation.

Un second essai, qui lui également n'a étế utilisé que pour la recherche, est l'essai scissométrique à base d'autoforage. Il s'agit, comme dans l'essai scissométrique (vane-test) traditionnel, de cisailler par rotation autour d'un axe vertical le sol intact, par un jeu de pales de très petites dimensions. On détermine directement à partir d'une courbe couple appliquérotation un module de cisaillement G, qui peut d'ailleurs différer de celui déduit de la sonde frottante, puisque cette dernière met surtout en jeu les propriétés verticales du sol, alors que ce sont les caractéristiques surtout horizontales qui sont sollicitées avec le scissomètre.

\section{3}

\section{Tassement d'une fondation superficielle isolée}

L'utilisation des différents modules tels que définis précédemment, peut être examinèe à la lueur du comportement d'une fondation superficielle, posée sur un massif de sol réputé homogène, dont l'épaisseur est très grande devant la dimension transversale de la fondation.

Lorsqu'on procède à un essai de chargement, celui ci est mené par paliers successifs de charge, maintenus le plus souvent une heure, mais certains essais spécifiques ont vu ce maintien porté à 24 heures. On obtient ainsi, en figure 28 , une courbe de chargement enveloppe qui n’est pas linéaire.

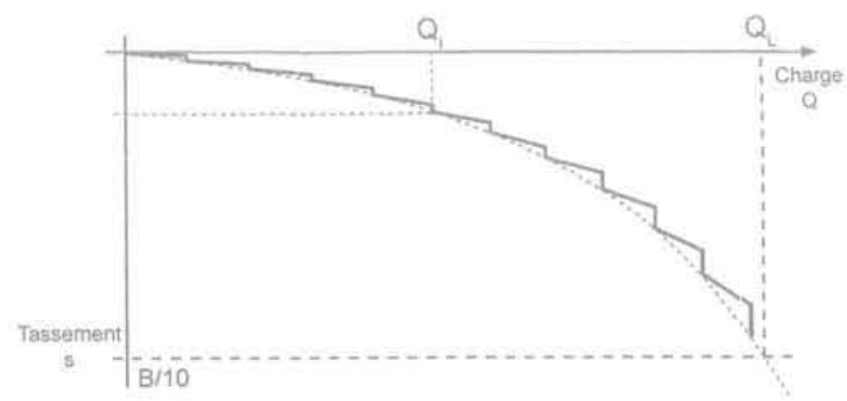

FIG. 28 Courbe charge-tassement d'une fondation superficielle.

Load-settlement curve for a shallow foundation. 
L'évolution du tassement en fonction du temps, qu'on appelle « fluage », pour une charge appliquée $Q_{\text {, }}$, peut résulter du drainage, de mouvements relatifs entre particules et de compression dite secondaire (laquelle correspond à un réel phénomène de fluage et à une modification de la structure des particules élémentaires du sol du fait de leur nature).

La courbe enveloppe n'est pas linéaire et la loi de fluage peut s'écrire $s_{j}(t)=s_{i}\left(1+\alpha_{i} \ln \frac{t}{t_{0}}\right)$, où $s_{i}$ est le tassement instantané sous $Q_{1}$ et $\alpha_{1}$ est un coefficient qui croît avec $Q$. Il est utile de rappeler que la charge limite $Q_{1}$ de la fondation de largeur $B$ est par définition celle pour laquelle l'enfoncement vaut $\frac{B}{10}$.

On constate que pour des charges $Q_{i}$ permanentes inférieures à $\frac{Q_{1}}{3}$, les valeurs $\alpha_{1}$ sont extrêmement faibles et la connaissance de s est largement suffisante pour estimer le tassement à long terme de la fondation superficielle, du moins pour les sols où cette dernière est raisonnablement envisageable. Dans les sols fins très déformables, cette réalité du " fluage » est telle qu'il y a lieu de l'étudier de près et la conception de fondations superficielles, quand elles sont envisagées, est délicate.

Si la courbe de tassement (avec 1 heure de maintien de la charge à chaque palier) est par exemple celle de la figure 28, celle qui serait obtenue avec des paliers extrêmement longs, intégrant le drainage pour un sol fin, aurait l'allure donnée à la figure 29 qui met en jeu les modules drainés et donc très inférieurs au module non drainés mobilisés pour la première.

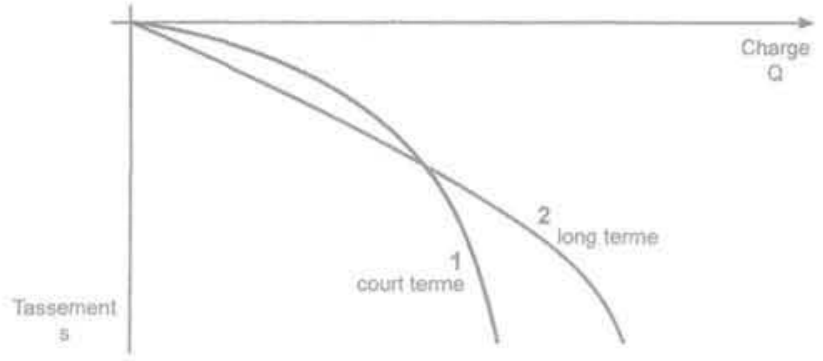

FG. 29 Tassement d'une fondation à court et long terme.

Short and long settlement for a shallow foundation.
Pour les sols granulaires et les sols fins non saturés, cette différence est très faible, et le comportement à long terme ne constitue pas une préoccupation importante, dans la mesure où pour les seconds il n'y a pas d'imbibition importante, voire de saturation, ce qui constitue un autre problème.

Deux approches sont couramment utilisées pour prévoir le tassement d'une telle fondation : l'approche élastique, courante à l'étranger et dont on a rappelé la formulation lors de l'examen de l'essai de chargement statique par une plaque circulaire, et l'approche pressiométrique en utilisant le module $\mathrm{E}_{\mathrm{M}}$.

- L'approche élastique repose sur l'expression $\mathrm{s}=\mathrm{C}\left(\frac{1-v^{2}}{\mathrm{E}}\right) \mathrm{qR}$ où $\mathrm{C}$ est un coefficient dépendant de la forme (élancement) et de la rigidité de la fondation ; pour une fondation circulaire $\mathrm{C}=\frac{\pi}{2}$ si elle est rigide, $C=2$ si elle est souple; $R$ est sa demi-largeur (s est alors le tassement au centre de la surface chargée). Elle est appliquée pour tout le domaine de contrainte admissible et $E$ est un module sécant moyen. Celui-ci est déterminé à partir d'essais de laboratoire, surtout le triaxial, en veillant à mesurer un module qui soit transposable au calcul à effectuer; il s'agira d'un essai drainé, si le sol concerné est un sol fin saturé.

Mais la mesure d'un module $\mathrm{E}$. de rechargement au pressiomètre, assimilé à un module élastique moyen, peut permettre un tel calcul, encore peu courant, mais qui ne peut convenir que pour des massifs où le comportement à long terme diffère peu de celui à court terme. Cette approche linéarise la courbe chargeenfoncement et ne peut rendre compte de la plus grande rigidité constatée aux faibles contraintes.

- L'approche pressiométrique introduit les deux comportements déviatorique et sphérique explicités précédemment. Dans le cas d'une fondation circulaire, on prend en compte dans une demi-sphère immédiatement sous la fondation une compression prépondérante du sol, à tendance oedométrique et, au-delà, dans l'espace restant, une prépondérance de cisaillement, à tendance déviatorique.

L. Ménard explicite deux composantes du tassement issues des théories de l'élasticité et de la consolidation, et corrigées empiriquement de coefficients rendant compte en particulier du caractère non élastique

TABLEAUVI Valeurs du coefficient rhéologique $\alpha$.

Value of rhéological coefficient $\alpha$.

\begin{tabular}{|c|c|c|c|c|c|c|}
\hline & Tourbe & Argile & Limon & Sable & Grave & Rocher \\
\hline Type & $\alpha$ & $E_{*} / p_{i} \quad \alpha$ & $E_{M} / p_{1} \quad \alpha$ & $E_{M} / P_{1}$ & $E_{M} / p_{1} \quad \alpha$ & $\alpha$ \\
\hline
\end{tabular}

surconsolidé

\begin{tabular}{lllllllll} 
ou très serré & - & $>16$ & 1 & $>14$ & $2 / 3$ & $>12$ & $1 / 2$ & $>10$ \\
\hline $\begin{array}{l}\text { normalementconsolidé } \\
\text { Ounormalement serré́ }\end{array}$ & 1 & $9-16$ & $2 / 3$ & $8-14$ & $1 / 2$ & $7-12$ & $1 / 3$ & $6-10$
\end{tabular}

ou normalement serré

sous consolidé altéré

et remanié ou lâche

$-7-9$

$1 / 2$

$5-8$

$1 / 2$

$5 \cdot 7$

$1 / 3$

très peu fracturé

normal

$1 / 2$

très fracturé

$1 / 3$

très altéré 
du sol. Il introduit, en particulier, le coefficient $\alpha$, dit coefficient rhéologique, censé relier le module de cisaillement et le module dénommé $E^{+}$, appelé module de compression uniaxiale, et qui pourrait être mesuré directement à l'œdomètre ; il est donc assimilé au module œdométrique, ce qui constitue une extrême simplification. Ce coefficient, une fois le type et la nature du sol connu, est défini par le rapport $\alpha=\frac{2(1+v) G M}{E^{+}}=\frac{E M}{E^{+}}$.

Pour un sol qui serait parfaitement élastique $\alpha=1$.

Une autre approche, proposée par L. Ménard, est donnée l'expression $\alpha \cong\left(\frac{E M}{E r}\right)^{1 / 2}$ qui comporte les deux modules pressiométrique et de rechargement définis auparavant. Un essai cyclique permet donc d'accéder à $\alpha$, que l'on retrouve égal à 1 , si le massif est élastique. II faut attirer l'attention, à cette occasion, sur la confusion très fréquente faite sur la valeur de $\alpha$, souvent identifiée au simple rapport des modules; ce n'en serait que la racine carrée (et en réalité, d'après L. Ménard, l'exposant affectant le rapport des modules est légèrement supérieur à 1/2).

Il faut cependant reconnaitre que cette confusion est entretenue si l'on examine l'expression empirique proposée dans le cadre de la théorie pressiométrique sensée fournir la cohésion non drainée $c_{4}$ à partir des caractéristiques pressiométriques.

$$
\text { Elle s'écrit : } p_{1}-p_{0}=c_{u}\left(1+\ln \frac{E M}{2 \alpha(1+v) c_{u}}\right) \text {. }
$$

Or, un sol supposé avoir un comportement élastoplastique, de module élastique $\mathrm{E}$ et de cohésion $\mathrm{C}_{\mathrm{u}}$ voit sa pression limite donnée théoriquement par $p_{1}-p_{0}=c_{u}\left(1+\ln \frac{E}{2(1+v) c_{u}}\right)$ qui montre que L. Ménard identifie $\mathrm{E}$ avec $\mathrm{E}_{\mathrm{M}} / \alpha$.

D'un point de vue pratique, le tableau VI rappelle les valeurs de $\alpha$ utilisées.

Les expressions des deux termes du tassement sont les suivantes, et elles sont sensées fournir le tassement à dix ans:

- tassement sphérique $\mathrm{s}_{\mathrm{s}}=\frac{1}{4,5} \frac{\alpha}{\mathrm{EM}} \mathrm{qR} \lambda_{\mathrm{s}}$, où le coefficient 4,5 résulte d'interprétations statistiques et empiriques et correspond à un coefficient de Poisson moyen d'environ 0,4, sans plus d'explication ;

- tassement déviatorique $s_{d}=\frac{1+v}{3 E M} q R_{0}\left(\lambda_{d} \cdot \frac{R}{R o}\right)^{\alpha}$ où $v$ est choisi égal à 0,33 , compte tenu du mode de détermination de $\mathrm{E}_{\mathrm{M}}$. qui le suppose. $\mathrm{R}_{0}$ est une dimension standard de $30 \mathrm{~cm}$, et la puissance $\alpha$ a été introduite pour rendre compte de la non-linéarité constatée lorsque $R$ augmente. On prend $R_{0}=R$ si $R \leq 0,30 \mathrm{~m}$. $\lambda$ et $\lambda_{\mathrm{d}}$ sont les deux coefficients de forme, liés au rapport de la longueur $\mathrm{L}$ à la largeur $2 \mathrm{R} ; 1<\lambda_{\mathrm{s}}<1,5$ et $1<\lambda_{\mathrm{d}}<2,65$ lorsque $1<\mathrm{L} / 2 \mathrm{R}<20$.

L'introduction pour $\mathrm{s}_{\mathrm{d}}$ d'un terme correctif non linéaire avec $\mathrm{R}$, traduit de fait le comportement non linéaire du sol ce qui est réaliste. Les contraintes diffusées en profondeur diminuant avec la distance à la fondation induisent des déformations locales de plus en plus faibles, correspondant donc à des modules de déformations locaux de plus en plus forts.

$$
\mathrm{S}_{\mathrm{d}}=\frac{1+v}{3} \mathrm{q} R \lambda_{\mathrm{d}}^{\alpha} \frac{1}{\operatorname{EM}\left(\frac{\mathrm{R}}{\mathrm{R}_{0}}\right)^{1-\alpha}} \text { où l'on fait apparaitre le }
$$

terme $E_{M}\left(\frac{R}{R_{0}}\right)^{1-\alpha}$, qui peut être assimilé à un module croissant avec $\mathrm{R}$. La valeur de $\mathrm{s}_{\mathrm{a}}$ reste néanmoins linéaire avec q.

Prenons l'exemple d'une semelle rectangulaire, de dimensions appréciables et assez lourdement chargée, sur un sol de bonnes caractéristiques, soit une argile graveleuse non saturée. Les données en sont $L=6 \mathrm{~m} ; \mathrm{R}=1 \mathrm{~m}$; encastrement $\mathrm{D}=1 \mathrm{~m} ; \mathrm{p}_{1}=1 \mathrm{MPa} ; \mathrm{E}_{\mathrm{M}}=10,5 \mathrm{MPa}$ et $\mathrm{q}_{\mathrm{a}}=0,3 \mathrm{MPa}$.

Les valeurs nécessaires au calcul sont $\alpha=\frac{2}{3}, \lambda_{5}=1,30$ et $\lambda_{d}=1,78$, qui fournissent $s_{s}=0,5 \mathrm{~cm}$ et $s_{d}=1,2 \mathrm{~cm}$, soit $\mathrm{s}_{\mathrm{total}}=1,7 \mathrm{~cm}$, valeur qu'il y a lieu d'augmenter de $10 \%$ comme la méthode le prévoit (ceci n'a pas été explicité ci-avant) du fait de l'encastrement. D'où $\mathrm{s}_{\text {final }}=1,9 \mathrm{~cm}$, soit le $1 / 100$ environ de la dimension transversale, ce qui est un ordre de grandeur courant entre tassement et dimension.

A l'issue de ces quelques exemples de calcul, on peut faire le constat suivant. Le dimensionnement économique d'une fondation superficielle consiste pratiquement à aboutir à une contrainte admissible permanente $\mathrm{q}_{\mathrm{a}}$ qui soit de l'ordre de grandeur de $\mathrm{p} / 3$. Il est alors instructif d'examiner la valeur $\mathrm{s} / 2 \mathrm{R}$, rapport du tassement à la largeur, qui vaut pour un sol homogène :

$$
\frac{\mathrm{s}}{2 \mathrm{R}}=\frac{\mathrm{q}_{\mathrm{s}}}{\mathrm{E}_{\mathrm{M}}}\left[\frac{\alpha \lambda_{\mathrm{s}}}{9}+\frac{1,33}{6} \lambda_{\mathrm{d}}^{\mathrm{d}}\left(\frac{\mathrm{R}}{\mathrm{R}_{0}}\right)^{\alpha-1}\right]=\frac{\mathrm{q}_{\mathrm{a}}}{\mathrm{E}_{\mathrm{M}}} \mathrm{K} \cong \frac{\mathrm{p}_{\mathrm{l}} \mathrm{K}}{3 \mathrm{E}_{\mathrm{M}}}
$$

En choisissant des couples $\left(\alpha . E_{M} / p\right)$ courants, on parvient à des valeurs de l'enfoncement relatif $S / 2 R$ montrant que dans les configurations les plus courantes, les valeurs de tassement ne sont guère préoccupantes, comme le tableau VII en témoigne.

Seules les fondations importantes, tant en longueur qu'en largeur, nécessitent des précautions vis-à-vis du tassement, et peuvent conduire à choisir des taux de travail inférieurs au tiers de la pression limite, comme il a été choisi dans ces exemples.

Lorsque les sols sont de nature variable en profondeur, les différents horizons sont caractérisés par des

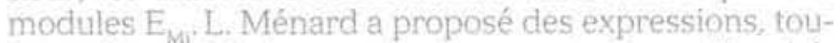
jours d'actualité, donnant les tassements respectifs sphérique et déviatorique, tenant compte de ces variations des modules. Il est, en principe, nécessaire de connaitre ces valeurs jusqu'à une profondeur valant huit fois la largeur de la semelle. La méthode est cependant heureusement applicable lorsque les investigations dans le sol n'atteignent pas ce niveau. C'est effectivement souvent le cas, justifié lorsqu'une bonne connaissance géologique et stratigraphique assure d'une relative homogénéité dans la déformabilité du massif, laquelle diminue d'ailleurs généralement avec la profondeur.

L'influence des horizons profonds est effectivement faible, et la majorité des déformations se produit sur une profondeur de 2 à 3 fois la largeur de la fondation, audelà de laquelle, les contraintes induites sont inférieures à $10 \%$ des contraintes apportées par la structure. 
TABLEAUVII Valeurs du rapport s/2R du tassement à la largeur de la fondation, et du tassement absolu. Value of ratio s/2R (settlement / width) and value of total settlement.

\begin{tabular}{|c|c|c|c|c|}
\hline & \multicolumn{2}{|c|}{$\begin{array}{c}\alpha=1 \frac{E M}{p_{r}}=16 \\
\text { (argile) }\end{array}$} & \multicolumn{2}{|c|}{$\begin{array}{c}\alpha=1 / 4 \frac{E M}{P_{f}}=6 \\
\text { (grave) }\end{array}$} \\
\hline & $\begin{array}{c}\text { semelle circulaire } \\
L / 2 R=1\end{array}$ & $L / 2 R=20$ & $L / 2 R=1$ & $L / 2 \mathrm{R}=20$ \\
\hline$\frac{R}{R_{0}}=1$ & $\begin{array}{l}\frac{1}{145} \\
\mathrm{~s}=0.4 \mathrm{~cm}\end{array}$ & $\frac{1}{62}$ & $\begin{array}{l}\frac{1}{72} \\
\mathrm{~s}=0,8 \mathrm{~cm}\end{array}$ & $\begin{array}{l}\frac{1}{55} \\
\mathrm{~s}=1,1 \mathrm{~cm}\end{array}$ \\
\hline$\frac{\mathrm{R}}{\mathrm{R}_{0}}=4$ & $\begin{array}{c}\frac{1}{145} \\
\mathrm{~s}=1,6 \mathrm{~cm}\end{array}$ & $\begin{array}{l}\frac{1}{62} \\
s=4 \mathrm{~cm}\end{array}$ & $\begin{array}{c}\frac{1}{169} \\
\mathrm{~s}=1,4 \mathrm{~cm}\end{array}$ & $\begin{array}{c}\frac{1}{126} \\
\mathrm{~s}=1,9 \mathrm{~cm}\end{array}$ \\
\hline
\end{tabular}

Ce constat est en accord avec les principes de calcul indirect des tassements, développés en sol homogène à partir d'autres types d'essai in situ tel le pénétromètre statique, où l'on ne s'intéresse qu'à une épaisseur de sol valant deux fois cette largeur.

Il convient de rappeler que la mise au point initiale de la méthode pressiométrique de calcul du tassement s'est appuyée sur des valeurs du module $\mathrm{E}_{\mathrm{M}}$ mesurées dans des forages réalisés en tarière à main, et donc dans des sols fins. Les calages expérimentaux de l'époque, il y a plus de quarante ans, ont concerné des fondations de dimensions tout à fait modestes (de l'ordre du mètre) dans des sols où de bons essais standards pouvaient donc être réalisés et avec des taux de travail moyens proches du tiers de la contrainte de rupture du sol sous la semelle. C'est-à-dire que l'on se trouvait dans des situations où consolidation et fluage étaient faibles devant le tassement total mesuré.

Des confrontations ultérieures ont bien montré, sur la base des méthodes de calcul alors proposées, que l'extension à de très grandes dimensions conduisait dans beaucoup de cas à des différences très importantes entre calculs et mesures, et ce d'autant plus que des sols compressibles et peu perméables étaient en cause. En revanche, pour des dimensions transversales très modérées $(<1,5 \mathrm{~m})$ et des rapports longueur sur largeur faibles, il est bien admis maintenant que la méthode fournit une estimation correcte du tassement différé à long terme, pour des coefficients de sécurité de 3, comme cela a été montré (Frank, 1991).

On doit citer ici, dans le cadre de la méthode pressiométrique, l'introduction d'un modèle de calcul de tassement de semelle non linéaire avec q (Combarieu, 1988) pour les besoins de la justification des fondations mixtes semelle/pieux sous charge verticale centrée. Dans une telle configuration, où la pointe du pieu, le frottement latéral (réciuit) et la semelle mobilisent simuitanément des efforts, il faut pouvoir séparer chacun des termes correspondants, et il s'avère nécessaire de procéder à une vérification en déplacement. Ce processus s'avère d'autant plus utile que la semelle ou le radier a une dimension importante. La pertinence de la justification passe par une modélisation non linéaire des lois efforts-déformation. C'est ce qui a èté fait dans une note d'information technique, où le modèle proposé revient à raidir fortement la courbe initiale enfoncement de la semelle/charge appliquée, sachant que, pour une contrainte valant la moitié de la contrainte de rupture de la semelle, le tassement obtenu est identique à celui obtenu par les expressions pressiométriques traditionnelles.

On peut se livrer à une comparaison simple portant sur la relation qui devrait relier le module $E_{M}$ au module élastique $\mathrm{E}$ (assimilé à $\mathrm{E}$, mesuré à l'essaj cyclique) pour parvenir à la même prévision.

Ainsi, dans le cas d'un massif homogène, semi-indéfini, pour une semelle de longueur L et de largeur 2R, l'égalité des tassements amène à écrire :

$$
2 \overline{\mathrm{P}}\left(\frac{1-v^{2}}{\mathrm{E}}\right) q \mathrm{R}=\frac{\mathrm{R}_{\mathrm{q}}}{\mathrm{EM}}\left[\frac{1,33}{3}\left(\lambda_{\mathrm{d}}\right)^{\alpha}\left(\frac{\mathrm{R}}{\mathrm{F}_{\mathrm{o}}}\right)^{\alpha-1}+\frac{\alpha \lambda_{\mathrm{s}}}{4,5}\right]
$$

oủ $\mathrm{P}$, coefficient sans dimension, dépend de l'élancement L/2R (comme $\lambda_{4}$ et $\lambda$ ) et est donné dans des tables (Giroud, 1972), v : coefficient de Poisson est pris égal à 0,33 comme pour le calcul de $\mathrm{E}_{\mathrm{M}}$.

Dans le cas d'une semelle circulaire:

$$
E_{M} / E=0,32\left(\left(\frac{R}{R_{0}}\right)^{\alpha-1}+\frac{\alpha}{2}\right) \text {. }
$$

Pour une semelle d'élancement 20 où $P=2,53$. $\lambda_{\mathrm{s}}=1,5$ et $\lambda_{\mathrm{d}}=2,65, \mathrm{E}_{\mathrm{M}} / \mathrm{E}=0,1\left[2,65^{\alpha}\left(\frac{\mathrm{R}}{\mathrm{R}_{0}}\right)^{\alpha-1}+0,75 \alpha\right]$, dont les valeurs numériques des inverses $E / E_{M}$ sont données dans les tableaux VIII.a et $b$.

Le rapport $E / E_{M}$ n'est évidemment pas constant, it augmente avec $\alpha$ décroissant et $L / 2 R$ croissant. Néanmoins, pour $\alpha=1$ qui correspond à une argile surconsolidée, ce rapport s'échelonne entre 2 et 3 , conforme à ce que l'on peut mesurer. Pour les autres valeurs de $\alpha$, en restant dans un domaine de dimension le plus courant, c'est-à-dire des largeurs $2 \mathrm{R}$ de 1 à $2,50 \mathrm{~m}$ soit $R / R_{0}$ variant de 2 à 4 , on peut constater pour les limons $(\alpha=2 / 3)$, des rapports $\frac{E}{E M}$ allant de 2,5 à 6 et pour les sables $(\alpha=1 / 3)$, de 4 à 14 .

On peut donc tout légitimement prévoir les tassements en utilisant les résultats de module obtenus par un essai cyclique dont le projet de norme existe d'ailleurs (Afnor, 1999). Cette approche est en outre justifiée puisque le module $E_{\text {r }}$ est déterminé lors de l'essai dans une plage de contrainte similaire à celle utilisée pour la détermination du module standard $E_{M}$.

On montre ainsi que tenter un calcul élastique en introduisant un module défini par $\frac{E M}{\alpha}$ ne peut pas convenir et donnerait des valeurs de tassements très pessimistes, puisque l'obtention de tassements identiques requiert de choisir une valeur $\mathrm{E}=\mathrm{kE}_{\mathrm{M}}$ en toute rigueur variable avec les dimensions de la semelle (les valeurs de k données par les deux tableaux sont très supérieures à $\frac{1}{\alpha}$ ). 


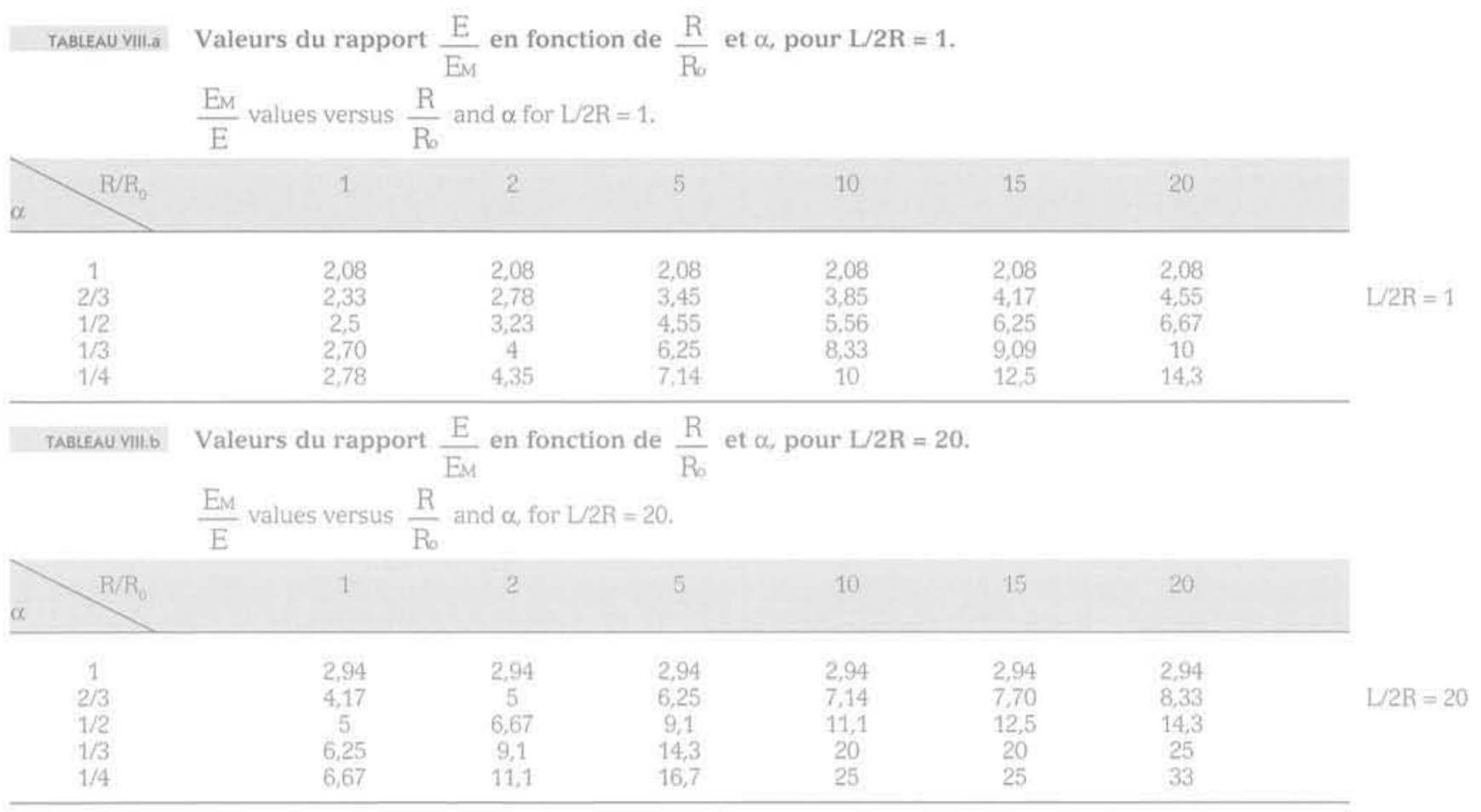

Il n'est donc pas étonnant que dans certains calculs aux éléments finis, les calculateurs introduisent maintenant à bon escient des modules définis par des expressions telles que $\mathrm{E}=2$ à 3 , voire 4 fois $\frac{\mathrm{Em}}{\alpha}$. Cela est parfaitement justifié et imposé par le constat de déformations mesurées plus faibles que celles déduites des prévisions utilisant la valeur $\frac{E M}{\alpha}$.

La sortie toute récente du volume 2 des symposiums PARAM 2002, ASEP-GI 2004 et FOND SUP 2003 permet, de façon plus complète et détaillée, une comparaison entre méthodes pressiométrique, élastique et par éléments finis, appliquées pour des prévisions de tassements pour des semelles de faibles dimensions transversales $(1,00 \mathrm{~m})$.

Dans la synthèse présentée (Droniuc, Magnan, 2004), la figure 5 en particulier montre pour $A_{\text {, }}$, semelle filante sur surface plane indéfinie, une parfaite identité entre calcul élastique classique (repéré Co élastique) et la méthode par éléments finis utilisée avec un modèle de comportement non linéaire (MCNL). Le pressiomètre (Co pressio) donne une valeur supérieure du $25 \%$. Les résultats sont identiques pour les cas $A_{2}$ (semelle filante enterrée, charge inclinée et excentrée), toutes méthodes confondues. Par contre, le cas de la semelle carrée est très contrasté, suivant que l'on se situe en surface ou en position enterrée.

Comme il a été montré pour le terme déviatorique $\mathrm{s}_{\mathrm{d}}$ du tassement, dans la théorie pressiométrique, uné meilleure concordance entre cette théorie et l'élasticité demande donc pour cette dernière d'introduire une nonlinéarité du tassement avec R en modifiant les valeurs du module E choisi, avec la dimension transversale de la fondation. Pour un massif de sol homogène caractérisé

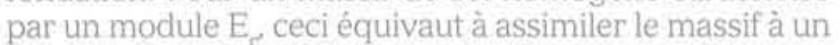
milieu où le module élastique croît avec la profondeur.

Aussi nous sommes-nous livrés à une étude paramétrique qui conduit à proposer la correction suivante, applicable aux fondations circulaires sur un massif homogène : celui-ci caractérisé par un module $\mathrm{E}_{r}$, mesuré ou estimé à partir de $\mathrm{E}_{\mathrm{M}^{\prime}}$ est assimilé à un massif où le module croit linéairement avec la profondeur, avec $E(z)=E_{r}(1+\lambda . z)$, où $\lambda=0,2(1-\alpha)$ où $\alpha$ est le coefficient rhéologique du sol. C'est une formulation analytique simple, qui peut sans doute être étendue aux fondations superficielles rectangulaires.

Dans la mesure où le sol possède déjà un module qui croît avec la profondeur, ce qui est le plus souvent le cas, et, si par simplification, on considère cette croissance linéaire avec $z$, de forme $E(z)=E_{1}(1+a z)$, la correction proposée y sera appliquée, conduisant à considérer une variation finale $E(z)=E_{r}[1+(a+\lambda) z]$.

Ainsi, la figure 30 donne les profils de module $E_{M}$ et de pression limite p mesuré sur un site naturel d'argile sableuse puis de sable calcaire, situé dans l'Orne, qui illustre bien l'augmentation, d'ailleurs rapide, de ces caractéristiques avec la profondeur.

Un autre exemple de cette croissance du module avec la profondeur est donné par un massif de sable de Fontainebleau de deux mètres d'épaisseur, mis en place par couches régulières de $30 \mathrm{~cm}$, et compacté

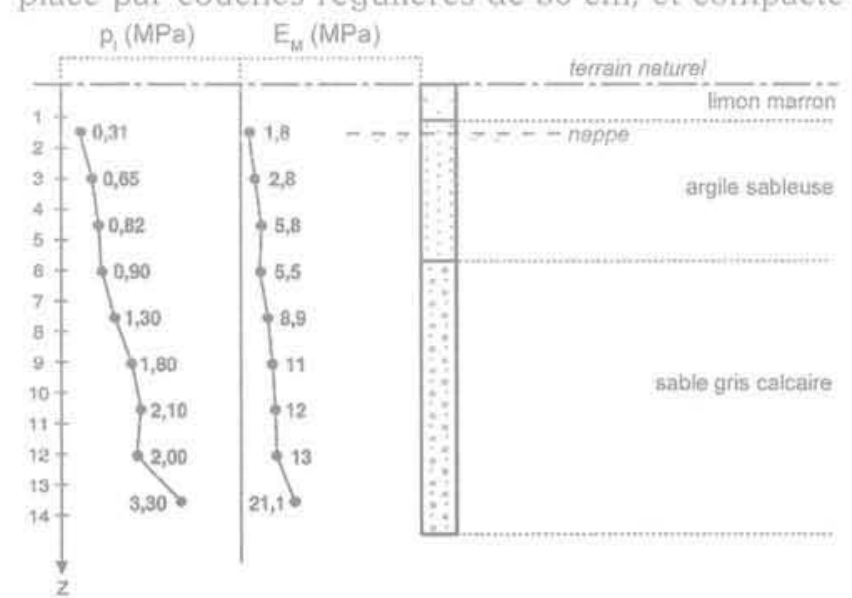

FIG. 30 Variations de $p_{1}$ et $E_{M}$ avec la profondeur dans un massif sableux. Variations of $p_{1}$ and $E_{M}$ with depth, in sandy soil. 
énergiquement par plaque vibrante assez lourde. Il s'agit d'un remblai de «fondation » destiné à recevoir une fondation superficielle, objet d'un essai de chargement statique. Pouvant être qualifié de très homogène, avec un poids volumique sec de $14,8 \mathrm{kN} / \mathrm{m}^{3}$ sur toute sa hauteur, ce massif a été l'objet d'essais de caractérisation mécanique montrant une forte augmentation des caractéristiques avec la profondeur.

Ainsi, à $0,70 \mathrm{~m}$, on a obtenu $\mathrm{p}_{\mathrm{l}}=635 \mathrm{kPa}, \mathrm{E}_{\mathrm{M}}=5,4 \mathrm{MPa}$, $\mathrm{E}_{\mathrm{r}}=11,6 \mathrm{MPa}$ et $\mathrm{E}_{\mathrm{d}}$ (module de déchargement) $=16 \mathrm{MPa}$. Dans le même forage, à $1,70 \mathrm{~m}$, ces valeurs deviennent respectivement $1515 \mathrm{kPa}$ pour $\mathrm{p}_{\text {, }} 15,4 \mathrm{MPa}$ pour $\mathrm{E}_{\mathrm{M}}$

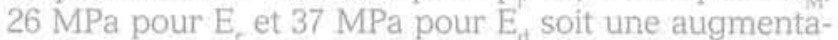
tion considérable sur $1,00 \mathrm{~m}$ de distance verticale. On notera d'ailleurs au passage que le rapport $\mathrm{E}_{\mathrm{r}} / \mathrm{E}_{\mathrm{M}}$ est relativement faible, de l'ordre de 2 , alors que pour des sites naturels de sable ce rapport est d'un ordre de grandeur très supérieur. Par contre, la valeur de 1, réputée dans la théorie pressiométrique qualifier un matériau de remblai, ce qui est le cas ici, n'est pas vérifiée.

On dispose alors des outils et abaques nécessaires (Giroud, 1973) pour calculer le tassement de la fondation, dans le cas d'une semelle circulaire souple. Celui de la semelle rigide étudiée, sera corrigé du terme $\frac{\pi}{4}$, ce qui constitue une approximation satisfaisante.

Les deux exemples qui suivent illustrent le cas d'une fondation circulaire posée sur une argile graveleuse puis un sable.

Le premier sol est caractérisé par $q_{n}=0,3 \mathrm{MPa}$, $E_{M}=10,5 \mathrm{MPa}, E_{r}$ est estimé à $3 E_{M} ; \alpha=2 / 3 ; v=0,33$. Le tableau IX.a donne le tassement pour divers rapports $R / R_{0}\left(R_{0}=0,3 \mathrm{~m}\right)$, avec la méthode pressiométrique, la méthode élastique avec $\mathrm{E}\left(=\mathrm{E}_{\mathrm{J}}\right)=31,5 \mathrm{MPa}$ et la méthode élastique corrigée où $E(z)=31,5\left(1+\frac{2}{3} z\right)$.

Le second est un sable de densité modérée, de mêmes pression limite et module $\left(\mathrm{q}_{\mathrm{a}}=0,3 \mathrm{MPa}\right.$, $\left.E_{M}=10,5 \mathrm{MPa}\right) ; \alpha=1 / 3$ et $E_{r}$ est estimé égal à $7 \mathrm{E}_{\mathrm{M}}$. Les tassements sont donnés dans le tableau IX.b.

Dans la mesure oủ la référénce est considérée comme le tassement calculé à partir de $\mathrm{E}_{\mathrm{M}}$, tassement intégrant implicitement une augmentation de la raideur du sol avec la diminution des contraintes induites en profondeur, la

\begin{tabular}{|c|c|c|c|c|c|}
\hline TABIEAU IX.G & \multicolumn{5}{|c|}{$\begin{array}{l}\text { Tassements calculés pour une argile } \\
\text { graveleuse. } \\
\text { Predicted settlement in gravel clay. }\end{array}$} \\
\hline $\mathrm{R} / \mathrm{R}_{0}$ & 2 & 4 & 10 & 20 & 50 \\
\hline $\mathrm{mm})$ & & & & & \\
\hline $\begin{array}{l}\text { ressiomètre } \\
(\mathrm{mm})\end{array}$ & 8,5 & 14,5 & 30,4 & 53 & 115,3 \\
\hline $\begin{array}{l}\text { lastique } \\
\text { (mm) }\end{array}$ & 8,1 & 16,1 & 40,5 & 81 & 202 \\
\hline lastique corrigé & 7,1 & 14 & 31,6 & 54,7 & 99,9 \\
\hline
\end{tabular}

TABLEAU IX.b Tassements calculés pour un sable. Predicted settlement in sand.

$\begin{array}{lllll}2 & 4 & 10 & 20 & 50\end{array}$

$\mathrm{s}(\mathrm{mm})$

\begin{tabular}{lccccc}
$\begin{array}{l}\text { pressiomètre } \\
\begin{array}{l}\mathrm{S}(\mathrm{mm}) \\
\text { élasticité }\end{array}\end{array}$ & 6 & 8,5 & 14,5 & 23,1 & 47,8 \\
$\begin{array}{l}\mathrm{s}(\mathrm{mm}) \\
\text { élasticité corrigée }\end{array}$ & 3 & 7 & 17,5 & 35 & 87 \\
\hline
\end{tabular}

valeur du tassement obtenue parallèlement à partir d'un module élastique $E_{r^{\prime}}$ issu de l'essai pressiométrique, peut donc être corrigée simplement pour parvenir à des ordres de grandeur identiques.

D'un point de vue pratique, il faut cependant reconnaître que, au devant d'une largeur croissante d'une fondation, le massif est en général non homogène, et ce pour deux raisons :

- si statigraphiquement il l'est, ses caractéristiques augmentent avec la profondeur : on le constate en permanence, avec la pression limite, le module de déformation, ou bien la cohésion non drainée par exemple. Ce point a déjà été souligné ;

- sinon, en profondeur, apparaissent des variations de faciès et de nature de matériaux, qui conduisent à dresser des modèles géotechniques qui sont des multicouches aux propriétés très contrastées.

Dans ces conditions, pour des fondations superficielles qui ont longuement retenu notre attention, l'application de la méthode pressiométrique est adaptée, puisqu'elle est capable d'intégrer ces variations de faciès. Mais l'examen précédent ne s'applique qu'aux sols où les phénomènes de consolidation sont faibles, sachant que, dans le cas contraire et pour les dimensions de fondation croissantes, il sera montré plus loin à quelles difficultés on est confronté.

On retiendra de cet examen de la prévision du tassement des fondations que le calcul pressiométrique est adapté mais qu'il convient de modérer le rôle et l'importance du coefficient rhéologique $\alpha$. On lui a attribué des vertus contradictoires qui amènent à des confusions fâcheuses. Il a un rôle extrêmement utile en rendant bien compte du tassement des fondations superficielles courantes. Les variations de sa valeur suivant la nature (ou type) de sol reflètent, on peut l'interpréter comme tel, un comportement propre à cette nature; elles rendent compte de la déformabilité naturelle et croissante des massifs avec la profondeur, et de l'aptitude plus ou moins grande du sol à diffuser les contraintes.

En aucun cas, on ne peut considérer le rapport $\frac{E_{M}}{\alpha}$ comme un module élastique et le rôle central que l'on fait jouer à ce terme n'a pas lieu d'être.

L'application d'une méthode élastique pour prévoir les tassements se heurte rapidement, pour des dimensions importantes, à l'impossibilité de calculs faciles, même en massif homogène, les outils existants et de simple portée étant limités à un nombre restreint de configurations. Seule une modélisation plus complexe du comportement permet raisonnablement d'aborder le problème. C'est ce qui est utilisé pour les grands radiers. Seules des fondations de dimensions transversales limitées, les plus courantes, avec $R / R_{0} \leq 5$, sont susceptibles, dans un massif homogène, d'un calcul élastique direct, où les corrections évoquées, liées à la dimension et qui s'imposeraient, restent modérées.

Pour terminer, il faut évoquer un autre facteur, à peine esquissé dans les pages précédentes, qui perturbe ces calculs et comparaisons et provient de l'anisotropie naturelle d'un sol. Du fait de son mode de formation et de son histoire, un massif présente des caractéristiques verticales et horizontales différentes. Les modules mesurés à l'essai triaxial ou à l'œdomètre concernent les propriétés verticales du sol, alors que ceux mesurés au pressiomètre mettent en jeu les propriétés horizontales. C'est donc une source d'erreur si 
l'on utilise un module de type pressiométrique, pour prévoir le tassement sous une charge verticale, fondation ou remblai d'apport. Il n'est pratiquement jamais tenu compte de cette réalité dans la résolution de ce type de problème.

On donne ci-dessous des valeurs usuelles du rapport $E_{h} / E_{v}$, issues de travaux sur ce sujet (Garnier, 1973): - sables très peu denses à très denses $0,4<\mathrm{E}_{\mathrm{h}} / \mathrm{E}_{\mathrm{v}}<1$; - argiles normalement consolidées $\mathrm{E}_{\mathrm{h}} / \mathrm{E}_{\mathrm{v}} \cong 1$;

- argiles surconsolidées à très consolidées $1<\mathrm{E}_{\mathrm{h}} / \mathrm{E}_{\mathrm{y}}<3$.

On sait résoudre en élasticité linéaire, certains problèmes de chargement sur sol anisotrope. Cinq paramètres élastiques, deux modules ( $E_{n}$ et $E_{v}$ ) et trois coefficients de Poisson, sont alors nécessaires : on conçoit donc que le recours à de telles solutions est rare.

Néanmoins et heureusement, l'incidence de l'amplitude de l'anisotropie reste faible, et une récente étude (Pouya, Reiffsteck, 2003) le montre, sous réserve d'admettre un modèle anisotrope un peu particulier qui permet de réduire le nombre des paramètres d'élasticité à trois au lieu de cinq.

Ainsi le tassement d'une semelle circulaire rigide de rayon $R$ posé sur un sol de caractéristique $E_{\psi}, E_{h}$ et v est donné par $\mathrm{s}=\left(\frac{E_{\mathrm{v}}}{E_{\mathrm{h}}}\right)^{1 / 4} \frac{\pi}{2} \frac{1-v^{2}}{E_{\mathrm{v}}} q R$ ou bien $\mathrm{s}=\left(\frac{\mathrm{E}_{\mathrm{h}}}{\mathrm{E}_{\mathrm{v}}}\right)^{3 / 4} \frac{\pi}{2} \frac{1-v^{2}}{\mathrm{E}_{\mathrm{h}}} \mathrm{qR}$.

Avec pour un sable dense de densité relative $\mathrm{Dr}=0,8$ où $\mathrm{E}_{\mathrm{h}} / \mathrm{E}_{\mathrm{v}}$ vaut 0,9 , le tassement obtenu à partir du pressiomètre $(\mathrm{E}=\mathrm{E})$ serait à corriger par 0,92. Ce correctif atteindrait 0,76 pour le même sable beaucoup moins dense ( $\mathrm{Dr}=$ $\left.0,4, E_{2} / E_{r}=0,7\right)$. On hésiterait cependant à prévoir une fondation superficielle telle quelle, sur ce dernier.

Pour une argile très fortement consolidée, le terme correctif serait de 2,28 , ce qui amplifie très fortement le tassement, dont on peut penser que la valeur absolue reste néanmoins acceptable car faible, compte tenu de I'histoire du massif.

\section{4}

\section{Tassement sous charge de grandes dimensions}

La transition entre une fondation superficielle et un radier ou remblai de grandes dimensions est progressive, et l'application pour les seconds des expressions donnant le tassement appliqué à la première se heurte immédiatement à des difficultés, qui ont déjà été citées: - non-homogénéité du massif en profondeur, et nature des sols concernés;

- le massif est, en effet, souvent de mauvaise qualité, compressible, peu perméable. Jusqu'à présent délaissés par les constructeurs, ces terrains reçoivent maintenant des équipements de forte emprise : remblais et voiries, station d'épuration, bacs-réservoirs. Avant d'y prévoir des fondations profondes, l'examen des conditions de fondations en surface s'impose, puisque certaines de ces constructions peuvent tolérer des déformations qui peuvent être importantes avant leur mise en service, voire après, mais dans ce cas avec des amplitudes qui doivent rester faibles. Il s'agit de cerner le mieux possible ces dernières et leur évolution dans le temps.
L'outil le mieux adapté à de telles prévisions est l'essai cedométrique, dont le principe a été développé précédemment, et qui reste d'une totale actualité. Cela nécessite, bien entendu, un modèle géotechnique suffisamment fiable qui comporte les niveaux extrêmes de la nappe, les poids volumiques des couches stratigraphiques identifiées, les indices des vides, les coefficients de compressibilité, les contraintes de surconsolidation, et l'évolution du fluage.

Il est d'ailleurs utile de rappeler à cette occasion la relation simple $e_{0}=w_{5} \frac{\gamma_{s}}{\gamma_{w}}$, liant l'indice des vides $e_{\alpha}$ la

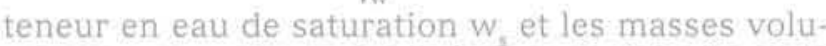
miques $\gamma_{5}$ et $\gamma_{w}$ des grains solides et de l'eau. La seule connaissance de $\mathrm{w}_{\mathrm{s}}$ permet donc d'accéder à $e_{\mathrm{q}}$, puis au poids volumique humide déjaugé $\gamma_{\mathrm{h}}^{\prime}=\gamma^{\prime}=\frac{\gamma_{\mathrm{s}}-\gamma_{\mathrm{w}}}{1+e_{\mathrm{n}}}$, nécessaire au calcul des contraintes verticales effectives en place $\sigma_{0}^{\prime}$

Enfin Cc, à défaut d'être mesuré, peut être également estimé à partir de $w_{\text {s }}$ avec des ordres de grandeur tels que $\mathrm{Cc}=0,7 \mathrm{w}_{\mathrm{s}}$ pour des argiles sableuses, $\mathrm{Cc}=\mathrm{w}_{\mathrm{s}}$ pour des argiles molles, $\mathrm{Cc}=1,25 \mathrm{w}_{5}$ pour des tourbes (dans ces matériaux, il faudra cependant veiller à la valeur de $\gamma$, qui peut atteindre largement moins de $20 \mathrm{kN} / \mathrm{m}^{3}$ au lieu des 26 à 27 pour les sols courants).

Cet ensemble de relations ou corrélations ne remplace certes pas les mesures des paramètres, mais il permet, si l'on utilise des méthodes autres de détermination du tassement, de procéder à des recoupements simples et de s'assurer du bon ordre de grandeur des valeurs trouvées.

On a vu que le tassement $\Delta \mathrm{L}$ d'une couche d'épaisseur L, s'écrit $\frac{\Delta L}{L}=\varepsilon_{z}=\frac{\sigma_{7}}{E_{o d d}^{\prime}}$, où $\varepsilon_{\text {ced }}^{\prime}$ varie avec la profondeur

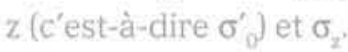

Une étude sérieuse relative aux sols compressibles et aux dispositions constructives à prévoir objectivement nécessite des moyens dont le coût est souvent sans commune mesure avec celui des travaux qu'on sera amener à entreprendre si cette étude est défaillante.

Dans un but d'économie, s'est fait jour, pour calculer les tassements, l'utilisation de plus en plus répandue (et enseignée), des résultats pressiométriques par le biais du module de déformation pressiométrique $\mathrm{E}_{\mathrm{M}}$ et ce, quelle que soit la nature du sol concerné. Cette pratique n'a pas de sens, c'est une erreur conceptuelle, et la concordance qui peut être observée et affichée entre tassements prévus et mesurés n'est que le fruit du hasard.

L'expression en cause donnant le tassement s'écrit $\mathrm{s}=\frac{\alpha \mathrm{H}}{\mathrm{E}} \mathrm{q}$, où $\mathrm{H}$ est l'épaisseur de la couche susceptible de tasser, $E_{M}$ et $\alpha$ le module pressiométrique et le coefficient rhéologique, q est la contrainte répartie appliquée. Le sol compressible n'étant l'objet que de tassements verticaux, sous la forme $\frac{\mathrm{S}}{\mathrm{H}}=\varepsilon_{2}=\frac{\mathrm{q}}{\mathrm{E}_{\mathrm{M}} / \alpha}, \mathrm{E}_{\mathrm{M}} / \alpha$ apparaît donc l'équívalent d'un module oedométrique et a effectivement ce statut dans la théorie pressiométrique. Ce module n'a cependant rien à voir avec le véritable module cedométrique, essentiellement variable avec la contrainte appliquée, comme nous l'avons montré dans cet article. L. Ménard a proposé une ( amélioration $»$ à cette formule en introduisant un 
terme correctif $\left(\beta=\frac{2 F}{3(F-1)}\right.$, avec $\beta=1$ si $\left.F \geq 3\right)$, fonction du coefficient de sécurité $F$ sous la forme $s=\frac{\alpha H}{E M} q \times \beta$ soit $\frac{s}{H}=\frac{q}{E M / \alpha \beta}$.

Cette notion de sécurité reste non définie et donc ambigué. On peut y trouver deux significations. La première est celle de la sécurité au sens de la stabilité des pentes concernant les talus des remblais mis en cuvre. C'est un problème néanmoins local et, pour un remblai de grande superficie, on peut imaginer un aménagement par des banquettes appropriées, amenant à une large sécurité, et ce quelle que soit la hauteur du remblai. La seconde interprétation, plus crédible et mieux adaptée, consiste à définir la valeur de F, comme le rapport entre la contrainte de rupture sous charge par poinçonnement et la contrainte q appliquée. Ainsi, une couche de sol de pression limite $p_{\text {, }}$ et une charge $q$, conduise à $\mathrm{F}=\frac{0,8 \mathrm{p}_{1}}{\mathrm{q}}$. Plus logique, cette approche n'en reste pas moins non fondée. Une couche d'argile molle de $1 \mathrm{~m}$ d'épaisseur, de $100 \mathrm{kPa}$ de $\mathrm{p}_{\text {, devrait en }}$ effet accuser un tassement calculé gigantesque pour $4 \mathrm{~m}$ de remblai, que l'on peut très bien édifier en aménageant les pentes des talus.

Quoi qu'il en soit, $\beta$ croît avec F décroissant et donc avec $q$ croissant. Le module « équivalent » $\frac{E_{M}}{\alpha \beta}$ décroît donc avec q et varie en sens inverse du véritable module œdométrique $\mathrm{E}_{\text {add }}^{\prime}$ qui, lui, croît avec q.

En définitive, ce qui est proposé à partir du pressiomètre se heurte à deux obstacles fondamentaux :

- $E_{M}$ est une valeur mesurée, constante pour la couche de sol considérée et traduisant un comportement non drainé et une déformation à court terme. En outre, une bonne mesure du module au pressiomètre dans les sols mous exige un soin particulier qui n'est pas toujours respecté. On peut même presque considérer que les matériels actuels ne sont guère adaptés à ces mesures ;

- la correction proposée qui équivaut à introduire une variation de ce module avec la contrainte, est contraire au phénomène physique du tassement œdométrique.

Une autre démonstration, s'il en est besoin, de la non-pertinence de l'utilisation du module pressiométrique peut être apportée en effectuant ce calcul par étapes successives, Il s'agit simplement par exemple pour un remblai de hauteur $h_{\text {f }}$ à édifier, d'en mettre en œuvre une hauteur $\frac{h_{r}}{2}$ dont le tassement final est alors sensé être donné par $\frac{\mathrm{S1}}{\mathrm{H}}=\frac{\alpha}{\mathrm{E}_{\mathrm{M} 1}} \frac{\gamma h_{\mathrm{r}}}{2}$.

Le sol compressible, de caractéristiques $\mathrm{E}_{\mathrm{Mr}}, \mathrm{p}_{11}$ et $\mathrm{C}_{\mathrm{u1}}$, voit, après consolidation, cette dernière croître à $\mathrm{C}_{\mathrm{u} 2}$ dont la valeur peut être estimée en adoptant une valeur réaliste de $\operatorname{tg} \varphi_{c u}$; on peut donc estimer les nouvelles valeurs, $p_{12}$ et $E_{M 2}$ du sol mou avec $E_{M 2}>E_{M 1}$.

Sous la nouvelle charge finale $\frac{\gamma \mathrm{hr}_{\mathrm{r}}}{2}$, qui marque l'achèvement du remblai, le tassement supplémentaire atteint $=\frac{\mathrm{s} 2}{\mathrm{H}-\mathrm{S} 1}=\frac{\alpha}{\mathrm{E}_{\mathrm{M} 2}} \frac{\gamma \mathrm{h}_{\mathrm{r}}}{2} ;$ ce qui conduit au tassement final $\frac{\mathrm{s}}{\mathrm{H}}=\frac{\mathrm{S}_{1}+\mathrm{s} 2}{\mathrm{H}}=\frac{\alpha \gamma \mathrm{h}_{\mathrm{r}}}{2}\left(\frac{1}{\mathrm{EM}_{1}}+\frac{1}{\mathrm{EM}_{2}}\left(1-\frac{\alpha \gamma \mathrm{h}_{\mathrm{r}}}{2 \mathrm{EM}_{1}}\right)\right)$. Cette valeur est inférieure à celle supposée obtenue après le chargement direct de la charge du remblai, à savoir $\frac{s}{H}=\frac{\alpha \gamma h_{1}}{E_{M 1}}$.

On obtiendrait de même des valeurs différentes avec un autre rythme de chargement du remblai.

On donne ci-après un exemple réel ayant fait l'objet d'un suivi important sur chantier (Lavisse, Schmitt, 2004). Il s'est agi au port de Rouen de prévoir à l'cdomètre et au pressiomètre, puis de mesurer, les tassements sous un remblai généralisé reposant sur plus de 15 mètres du sol compressible. Ces résultats ont montré la très bonne concordance entre mesures et prévisions traditionnelles à l'cedomètre, et la médiocrité des prévisions fournies par pressiomètre s'avérant quatre fois plus petites que les mesures.

Nous nous sommes intéressés sur le site en question à une couche de limon, normalement consolidée, de 1 mètre d'épaisseur, située à 5 mètres de profondeur avec une nappe (mal précisée) détectée vers 2,50 à 3 mètres. Les données mesurées sont les suivantes: $E_{M}=3000 \mathrm{kPa} ; \alpha=2 / 3 ; p_{1}=250 \mathrm{kPa} ; w=0,88 ;$ $C \mathrm{c}=0,73 ; \mathrm{e}_{0}=2,30 ; \sigma_{0}(5 \mathrm{~m})=60 \mathrm{kPa} ; \mathrm{q}=106,6 \mathrm{kPa}$.

Le calcul à l'œdomètre fournit $\mathrm{s}=0,1$ mètre, donnant $E_{\text {ad }}^{\prime}(106,6)=1066 \mathrm{kPa}$; de même $E_{\text {œC }}^{\prime}(0)=$ $623 \mathrm{kPa}$.

Le pressiomètre fournit $\mathrm{s}=0,025 \mathrm{~cm}$, soit quatre fois moins que la valeur précédente, ce qui recoupe le rapport de 4 trouvé pour l'épaisseur globale du sol compressible.

Si la charge de remblai q double, ce rapport des tassements calculés descend à 3 .

On peut certes évoquer, dans cet exemple, un rapport
$\frac{\mathrm{E}_{\mathrm{M}}}{\mathrm{p}_{\mathrm{I}}}$ élevé, de 12 , qu'une mise en œuvre plus conforme lors des essais pressiométriques aurait sans doute plus logiquement ramené à 8 par exemple. Le rapport des tassements ne passerait qu'à 3 .

Un deuxième exemple concerne les remblais de la liaison A29, au nord, pont de Normandie, au sud, qui traverse la large vallée de la Seine au Havre. Les franchissements multiples, canal de Tancarville, route industrielle, canal central maritime et route de l'estuaire, ont conduit à réaliser des remblais de 4 à 15 mètres de hauteur. Édifiés sur des sols plus ou moins compressibles et d'épaisseurs variables mais limitées à 6 mètres, les prévisions de tassements ont été à juste titre uniquement faites par la méthode œdométrique.

La montée des remblais, par étapes pour les plus hauts, a été accompagnée d'un suivi des tassements et des surpressions interstitielles dont les évolutions dans le temps ont gouverné les phases successives de la construction.

Compte tenu des variations d'épaisseur et de caractéristiques des sols, les différences maximales de $30 \%$ constatées entre prévisions et mesures permettent de conclure à une concordance tout à fait correcte, pour les dix profils installés (sur environ $3 \mathrm{~km}$ ), avec des tassements finaux variant entre 60 et $100 \mathrm{~cm}$, pour les hauteurs citées.

Si on se livre à de tels calculs à l'aide des essais pressiométriques dont on dispose, en particulier au droit des 
ouvrages d'art, on est rapidement confronté à des difficultés d'application des formules pressiométriques. On ne peut en effet supposer une mise en œuvre d'un seul tenant, ce qui nécessite donc d'introduire le coefficient. correctif $\beta$; l'instabilité étant manifeste, le tassement calculé est alors gigantesque et n'a aucune signification. Il faut donc tenir compte des phases successives et l'amélioration du sol support entraine donc une augmentation du module $\mathrm{E}_{\mathrm{M}}$ concerné. Si l'on effectue un tel calcul, en tenant compte du phasage effectivement suivi, à savoir le respect à chaque étape d'une sécurité F de 1,5 le coefficient $\beta$ vaut 2 et dans ces conditions, sans même faire varier $E_{M}$ les tassements ainsi calculés sont de l'ordre de la moitié de ceux mesurés.

Un troisième et dernier exemple que nous avons eu à expertiser concerne la fondation de réservoirs de diamètres et charges très variables, reposant par l'intermédiaire d'un remblai stabilisé sur des argiles molles moyennement à faiblement organiques et très plastiques.

Les diverses configurations de charges prévues ont amené à prévoir une amélioration des sols mous par des colonnes ballastées, conduisant à des tassements annoncés de $5 \mathrm{~cm}$ seulement pour les charges les plus lourdes. Ces valeurs, malheureusement déduites d'essais pressiométriques, ont résulté de deux approches : la première utilisant le module " cdométrique ) $E_{M} / \alpha$, tel que prévu par L. Ménard, la seconde en utilisant à partir de $E_{M}$ les corrélations présumées permettre d'accéder à un module de type $\frac{E^{\prime}\left(1-v^{\prime}\right)}{\left(1+v^{\prime}\right)\left(1-2 v^{\prime}\right)}$, véritablement cedométrique.

Les réservoirs ainsi fondés ont malheureusement accusé rapidement des déformations tout à fait inadmissibles atteignant, pour les plus lourds d'entre eux, des valeurs de près de $30 \mathrm{~cm}$, insupportables,

Une analyse faite après coup, à partir d'abord de la seule connaissance de la teneur en eau, puis des caractéristiques mesurées $\left(\gamma_{h^{\prime}}, C c . ..\right)$ a tout à fait été cohérente avec les mesures effectuées et montré le caractère très optimiste d'une prévision établie à partir du seul essai pressiométrique.

Dans la mesure où l'on souhaite néanmoins tirer profit de la valeur de $E_{M}$ pour effectuer un tel calcul de tassement, il y a lieu d'être extrêmement vigilant sur les "manipulations » auxquelles on se livre à partir de la valeur de $\mathrm{E}_{\mathrm{M}}$

Une approche plus logique devrait être la suivante : - le module pressiométrique $\mathrm{E}_{\mathrm{M}}$ est considéré comme un module sécant pour la plage $0-p / 2$. On en déduit un module cyclique $\mathrm{E}_{\mathrm{r}}$ (ou $\mathrm{E}_{\mathrm{u}}$ ) non drainé, $\mathrm{E}_{4}=\mathrm{n} \mathrm{E}_{\mathrm{M}}$. Le coefficient $\mathrm{n}$ est en principe supérieur à 1 (tableau $\mathrm{V}$ ) mais le mode opératoire de réalisation du forage d'essai peut conduire à $n \leq 1$. Ainsi le battage direct de la sonde de mesure pressiométrique (comme cela peut être pratiqué malheureusement) peut fortement surestimer $\mathrm{E}_{\mathrm{M}}$; cela semble avoir été le cas dans l'exemple cité sur le port de Rouen :

- on peut tenter ensuite d'estimer un module drainé $E^{\prime}$ tel que $\mathrm{E}^{\prime}=\mathrm{m}$ Eu où $\mathrm{m}<1$ (tableau IV.b) ;

- enfin le module œdométrique sera estimé par $E_{\alpha d}^{\prime}$ $\left(p_{1} / 2\right)=E_{\text {œd }}^{\prime}\left(p_{1} / 2\right)=\frac{\left(1-v^{\prime}\right) E^{\prime}}{\left(1+v^{\prime}\right)\left(1-2 v^{\prime}\right)}$ où v' est évidemment à fixer ; ce sera le module œdométrique sous la contrainte appliquée $\mathrm{p}_{\mathrm{l}} / 2$. Ce module ainsi déterminé est néanmoins constant ; pour tenir compte du com- portement du sol, on peut définir un module variable $\operatorname{par} E_{c \alpha d}^{\prime}\left(\sigma_{2}\right)=E_{\text {cd }}^{\prime}\left(p_{1} / 2\right)=\frac{2 \sigma_{2}}{p} \frac{\ln \left(1+\frac{p}{2 \sigma_{0}^{\prime}}\right)}{\ln \left(1+\frac{\sigma_{2}}{\sigma_{0}^{\prime}}\right)}$.

On se rapproche donc de la méthode cedométrique classique nécessitant d'apprécier $\sigma_{\sigma}^{\prime}$ ce que la méthode pressiométrique demande pour la détermination de la pression limite nette. On remarquera que $E_{\text {ced }}^{\prime}(0)=$ $E_{\text {ced }}^{\prime}\left(p_{1} / 2\right) \ln \frac{2 \sigma_{0}^{\prime}}{p_{1}} \ln \left(1+\frac{p l}{2 \sigma_{0}^{\prime}}\right)$, dont la valeur devrait être proche de $\frac{\sigma_{0}^{\prime}\left(1+e_{0}\right)}{0,435 \mathrm{Cc}}=E_{\text {ord,i }}^{\prime}$ ce qu'il sera utile de vérifier, même approximativement.

Cette démarche ne garantit cependant pas la validité du résultat du fait des nombreux coefficients ou hypothèses faites, une légère surconsolidation pouvant en outre exister.

Dans l'exemple déjà cité à Rouen, à $5 \mathrm{~m}$, $E_{\text {ced }, i}^{\prime}=\frac{60(1+2,30)}{0,435 \times 0,73} \cong 623 \mathrm{kPa}$; si l'on choisit $\mathrm{n}=1, \mathrm{~m}=$ 0,5 et $v^{\prime}=0,30$, alors $E_{\text {cod }}^{\prime}(p / 2)=2025$, et $E_{\text {cod }}^{\prime}(0)=$ $1094 \mathrm{kPa}$, soit une valeur un peu inférieure au double de $E_{\text {ret. }}^{\prime}$. Ces corrections conduisent donc, dans le cas présent, mais le choix des coefficients est discutable, à un tassement estimé à partir du pressiomètre d'environ la moitié de celui donné par l'œedomètre. C'est une démarche de type analogue à laquelle se sont d'ailleurs livrés J. Lavisse et P. Schmitt.

Cette démarche concernant les sols très compressibles et, par simplification, normalement consolidés, peut être également appliquée à toutes les autres natures de sols. Même si les tassements présumés auxquels on s'attend ont une amplitude faible, ils peuvent s'avérer problématiques vis-à-vis de l'interaction avec les ouvrages fondés.

Aussi nous conseillons la démarche suivante : pour les sables et graves très perméables on admettra $E_{4}=E^{\prime}$ et $v^{\prime}=0,25 ; E_{n}$ est évalué à partir de $E_{M}$ par $E_{u}=E_{r}=$ $7 \mathrm{E}_{\mathrm{M}}$ (tableau $\mathrm{V}$ ) d'où $\mathrm{E}_{\text {oed }}^{\prime} \cong 8 \mathrm{E}_{\mathrm{M}}$. Ceci équivaut à réduire de moitié ou de près du tiers les tassements déterminés en utilisant $\alpha(=1 / 4$ ou $1 / 3)$ et $E_{\mathrm{M}}$. Pour les limons, tels les limons de plateaux, les altérations du schiste, avec $E_{u} / E_{M}=4$, et $v^{\prime}=0,30$, s'ils ne sont pas saturés, en admettant $E^{\prime}=\frac{E_{i}}{1,2}$, alors $E_{\text {oed }}^{\prime}=4,5 E_{M}$, soit une réduction par 2 à 2,5 du tassement obtenu avec $\alpha(1 / 2$ à $2 / 3)$ et $E_{M^{\prime}}$.

Enfin, dans les argiles surconsolidées à très surconsolidées, on pourra estimer ces déformations à l'aide d'un module cedométrique de l'ordre de $4 \mathrm{E}_{\mathrm{M}}$ (au lieu de $=\frac{E_{M}}{\alpha}=\frac{E_{M}}{2 / 3}$ ) et de $5 E_{M}$ (au lieu de $E_{M}$ ), soit des déformations en moyenne 2,5 à 5 fois plus faibles.

Deux exemples permettent d'étayer de telles propositions. A l'occasion de l'étude des remblais d'accès aux ouvrages d'art, passages supérieurs, franchissant l'autoroute A29 en Normandie, le maitre d'œuvre avait souhaité disposer des tassomètres à la base de ces remblais. Ces accès d'environ $6 \mathrm{~m}$ de hauteur reposent sur des limons de plateaux, très légèrement compressibles sur environ $5 \mathrm{~m}$ et auxquels succèdent des argiles à silex, de décalcification de la craie, très compactes. 
L'estimation des tassements, pour $\sigma_{2}=120 \mathrm{kPa}$, avec $\alpha$ $=0,5$ et $\mathrm{E}_{\mathrm{M}}=3000 \mathrm{kPa}$ fournit $10 \mathrm{~cm}$, ramenés à $6 \mathrm{ou}$ $7 \mathrm{~cm}$ pour tenir compte de la géométrie du remblai (largeur limitée, talutage à la culée). Les mesures réalísées n'ont jamais dépassé 2 à $3 \mathrm{~cm}$.

Dans le Nord de la Seine-Maritime, se posait le comportement à long terme d'une argile secondaire du Gault, altérée et très surconsolidée, non saturée, de $5 \mathrm{~m}$ d'épaisseur supportant un remblai routier de $6 \mathrm{~m}$. A partir des caractéristiques mesurées au pressiomètre $p_{1}=0,6 \mathrm{MPa}, E_{M}=5 \mathrm{MPa}$ et $\alpha=1$, le tassement prévu $\alpha \mathrm{H} \sigma_{2}$

EM

donne $12 \mathrm{~cm}$, ce qui apparait excessif. On a admis en réalité que celui-ci n'excéderait pas 3 à $4 \mathrm{~cm}$, plus conforme à ce à quoi une approche traditionnelle et cedométrique, menée par ailleurs, pouvait conduire. Un début de suivi en place par simple plaque et pige a permis de mesurer des valeurs très inférieures à ces derniers chiffres.

Il faut souligner, pour ces sols, peu compressibles, que les propositions faites conservent une valeur constante au module proposé ; cette simplification est ici justifiée, du fait des valeurs faibles des déformations; les exemples numériques étudiés lors de l'examen de l'essai cedométrique l'ont d'ailleurs parfaitement montré.

\section{5}

\section{Déformation latérale des ouvrages de soutènement souples et des pieux}

Dans les situations où la déformée latérale de ce type d'ouvrages est en jeu, on dispose de trois types d'approche pour apprécier l'amplitude des mouvements. Ces approches se sont sophistiquées avec les exigences imposées, rendant nécessaires des méthodes de prévision plus sûres, qu'il faut sans cesse confronter à la réalité afin d'en améliorer les performances et la pertinence.

Les méthodes les plus simples sont déjà anciennes, et il n'y était même pas question de module du sol. Elles ont été très largement utilisées car étant les seules, et consistent à calculer la déformée d'un élément de poutre d'inertie donnée soumis à des effets appliqués en poussée ou en butée, résultant de calculs à la rupture, et assortis de coefficients de sécurité appropriés. Le choix des profilés de palplanches et l'adjonction d'une nappe de tirants permettaient de répondre facilement aux exigences de déformations, les calculs de celles-ci pouvant s'avérer cependant ardus car nécessitant des hypothèses simplificatrices du fait de l'hyperstaticité de la poutre en présence de tirants. On doit dire que cette pratique pouvait convenir pour des ouvrages souvent situés en zone vierge sans difficultés pour l'environnement, avec une possibilité de certaines déformations non contraignantes.

L'application à l'urbanisme de la technique des parois moulées pour la réalisation de grandes fouilles, a amené d'évidentes exigences de déformation, sinon difficiles à respecter, du moins plus difficiles à justifier, tout en respectant des critères économiques. La méthode précédente de calcul s'est vite révélée inadaptée et la déformabilité du sol a été introduite à juste titre dans le calcul avec la notion de coefficient (ou module) de réac- tion consistant à idéaliser mathématiquement un comportement du sol, qui ne peut être justifié physiquement, mais permet dans de nombreux cas de figure une approche suffisante et correcte des déformations.

Cette méthode permet d'intégrer dans le modèle de calcul, une loi d'interaction sol-structure, qui consiste à assimiler le sol en contact avec l'écran souple à une série de ressorts horizontaux, indépendants les uns des autres. Dans ces conditions on a une relation linéaire à un niveau $z$ donné $p=k_{n} y$ entre pression appliquée $p$ et déplacement $y ; k_{h}$ est appelé coefficient de réaction (horizontal).

Comme on l'a dit, cette proportionnalité n'existe pas, et il suffit pour s'en convaincre de considérer le cas d'une semelle souple puis rigide, sur un massif élastique. Dans le premier cas, sous une pression p uniforme, l'enfoncement au centre vaut $2\left(1-v^{2}\right) \frac{p R}{E}$ pour $\frac{4}{\pi}\left(1-v^{2}\right) \frac{p R}{E}$ sur le bord. En ces points respectifs les rapports pression/enfoncement varient donc de $\frac{2}{\pi}$. Dans le second cas de la plaque rigide, l'enfoncement $\frac{4}{\pi}\left(1-v^{2}\right) \frac{p R}{E}$ est uniforme avec une pression $p / 2$ au centre et infinie sur les bords ; le coefficient de réaction varie donc dans un rapport infini. Par contre, si on " globalise » cette notion à la plaque entière, le tassement moyen de la fondation souple s'écrit $\mathrm{s}_{\mathrm{m}}=\frac{1-\mathrm{v}^{2}}{\mathrm{E}} \frac{16}{3 \pi} \mathrm{pR}$, et celui de la plaque rigide $\mathrm{s}_{\mathrm{M}}=\frac{1-v^{2}}{\mathrm{E}} \frac{\pi}{2} \mathrm{pR}$, d'où $\frac{\mathrm{s}_{\mathrm{m}}}{\mathrm{p}}=1,70$ (ou 1,57) $\frac{\left(1-v^{2}\right) R}{E}$

L'extension de cet examen à une semelle rectanqulaire, montre, pour la largeur donnée $\mathrm{R}$, une modification du coefficient numérique de l'expression, qui rapidement atteint une valeur fixe avec l'élancement de la semelle. Ceci justifie, en particulier pour les pieux soumis à efforts horizontaux, l'assimilation de tronçons homogènes, liés à la stratigraphie et dotés de coefficients de réaction constants.

Ces coefficients, appelés aussi modules sont estimés dans les méthodes à partir, non pas de modules élastiques, mais de modules pressiométriques.

Pour les pieux, les valeurs de $\mathrm{k}_{\mathrm{h}}$ résultent directement des formules de tassement des fondations superficielles examinées dans un chapitre précédent. Elles sont tout à fait légitimes, les pieux ayant des dimensions transversales de l'ordre de grandeur $(0,50 \mathrm{~m}$ $2,00 \mathrm{~m}$ ) de celles pour lesquelles on a vu que la méthode était tout à fait appropriée aux semelles.

Pour les écrans souples, la détermination est plus complexe du fait d'une dimension transversale de l'écran très importante et très largement supérieure à la hauteur de l'écran. L. Ménard a proposé d'utiliser le même processus résultant du tassement des semelles, mais en considérant ici des semelles dont les largeurs sont des fractions de la hauteur de l'écran; la longueur de ces semelles équivalentes est celle de l'écran. On parvient de la sorte à exprimer le coefficient de réaction par l'expression $\frac{1}{k_{\mathrm{H}}}=\frac{1}{E_{M}}\left(\frac{\alpha a}{2}+0,133(9 \mathrm{a})^{\alpha}\right)$, où a est une largeur d'un élément supposé rigide. Cette approche est détaillée dans des recommandations (LCPC, 1985). 
L'exécution d'une paroi souple s'accompagne le plus souvent de tirants précontraints, pour lesquels la mise en tension met en œuvre des mécanismes de comportement du sol, très mal reflétés par l'utilisation des modules de réaction définis ci-dessus par une simple formulation pressiométrique. De tels constats sur des ouvrages en vraje grandeur ont conduit à mettre en place une troisième génération de calculs, avec les éléments finis, permettant d'édicter des règles pratiques autorisant l'usage des méthodes aux modules de réaction.

Le calcul par éléments finis, de plus en plus utilisé est conceptuellement meilleur, et les fortes exigences en site urbain en justifient l'utilisation. La complexité est si forte qu'il est extrêmement difficile de transposer un cas de figure à un autre.

Le choix d'une courbe effort-déformation peut consister en un modèle élastoplastique avec un module élastique $\mathrm{E}$, ou en un modèle plus complexe non linéaire, de type hyperbolique nécessitant entre autres paramètres la fixation d'un module de référence.

Le choix du module élastique se heurte aux questions soulevées à l'occasion des semelles. Trois publications récentes s'avèrent tout à fait significatives à cet égard, Les deux premières (Nguyen Phuong et al., 2005) relatent la comparaison entre prévisions et suivis pour deux parois moulées comportant une rangée de butons pour l'une et un lit de tirants pour l'autre. Dans le premier cas à Rouen, il y a bonne concordance entre mesure et calcul, avec un choix des modules élastiques qui ont été fixés égaux à $\mathrm{E}_{\mathrm{M}} / \alpha$; on peut constater que le rapport du déplacement maximal de $12 \mathrm{~mm}$ à la hauteur soutenue, en phase finale atteint environ $2.10^{-3}$. Pour le second cas à Calais, on a également obtenu une bonne concordance entre mesures de déplacement et prévision. Après mise en service, la valeur moyenne mesurée en tête atteint $6 \mathrm{~cm}$ pour une prévision identique, avec un rapport flèche/hauteur soutenue de $6 \mathrm{~cm} / 22 \mathrm{~m}$, soit près de $3.10^{-3}$. Les modules dans les sables flandriens concernés ont été choisis à $3 E_{M}(\alpha=1 / 3)$, et pour l'argile des Flandres à 3 ou $4 E_{M e}$ soit bien plus que ne le donnerait $E_{M} / \alpha$ avec $\alpha=2 / 3$. L'influence du module de cette argile n'intéressant que la partie de paroi profondément fichée n'est d'ailleurs pas très forte sur les résultats du calcul.

La bonne adéquation entre déformée et modules obtenus en choisissant $\mathrm{E}_{\mathrm{M}} / \alpha$ ne doit pas amener à conclure que cette option puisse être générale. Dans le cas présent, et surtout dans le second, la flèche en tête est très appréciable et acceptable. Si l'on s'était imposé une flèche beaucoup plus faible, une inertie plus importante de la paroi, et peutêtre un niveau de tirants supplémentaires, eussent été nécessaires. Le rapport flèche/hauteur soutenue plus faible nécessiterait par contre le choix d'un module élastique plus raide pour justifier l'ouvrage.

La troisième publication (Serrai et al., 2005) concerne une paroi moulée à Colombes, maintenue par deux niveaux de tirants précontraints. Les différents calculs, auxquels les auteurs se sont livrés pour tenter de retrouver les déformées aux différentes phases essentielles de la construction, ont conduit dans ce cas à devoir adopter des valeurs de module atteignant jusqu’à $4 \mathrm{E}_{\mathrm{M}} / \alpha$. Les ordres de grandeur de la déformée maximale, a savoir $5 \mathrm{~mm}$ en phase $\mathrm{n}^{\circ} 5$ pour la paroi sud, ne sont d'ailleurs que d'environ $5.10^{-4}$ fois la hauteur soutenue, ce qui est bien plus faible que pour les deux écrans précédents et explique la mise en jeu de modules beaucoup plus raides.
Les fortes variations de la déformée d'un rideau souple, sur toute sa hauteur conduit donc pratiquement inévitablement à introduire des lois non linéaires pour lesquelles il s'agit de fixer les paramètres essentiels déterminants, soit un module initial tangent soit un module sécant caractéristique.

L'évocation d'un coefficient $\mathrm{k}$ à appliquer au terme $\underline{E}$ et lié au niveau de déformation souhaité ne doit $\alpha$ pas non plus faire oublier que la dimension du rideau, avec les largeurs de semelles fictives considérées, intervient également par le biais de ce coefficient $\mathrm{k}$; il intègre donc deux aspects : non-linéarité et dimension. Pour ce deuxième aspect, le parallèle est à faire avec ce qui a été examiné pour les fondations superficielles.

Ce type d'ouvrage s'accompagnant de terrassement devant l'écran, on n'oubliera pas de s'intéresser aux paramètres de déformabilité des sols restant en place, mais ayant subi ces déchargements. Cet aspect du comportement constitue un élément supplémentaire qui rend encore plus complexe le dimensionnement d'une paroi. Enfin, il ne faut pas omettre l'influence de l'état initial des contraintes dans le massif. Son rôle sur la déformabilité est important et sa connaissance doit accompagner celle des modules.

En définitive, on peut dire que chaque paroi à justifier, au niveau des déformations, doit faire l'objet d'une attention très particulière, et s'il est une technique de travaux géotechniques où la méthode observationnelle, avec le dimensionnement interactif qu'elle implique, s'avère tout à fait appropriée et fortement recommandée, c'est bien celle de la construction des écrans souples.

\section{6}

\section{Conclusion}

L'examen de la notion de module en géotechnique s'avère trouver un intérêt dans les conséquences de l'application de cette notion aux problèmes les plus courants que les géotechniciens ont à résoudre. Dans les sols, la non-linéarité des déformations avec les efforts appliqués est un constat, que ce soit lors du cisaillement qui s'accompagne d'une baisse des modules ou lors de la compression oú les modules croissent.

On retiendra de cet examen, que pour les fondations superficielles et dans les sols où les phénomènes de consolidation sont faibles, l'utilisation de la méthode pressiométrique est tout à fait acceptable, soit directement sous la forme qui lui a été donnée par L. Ménard, soit indirectement sous une forme élastique en utilisant soit la corrélation reliant le module cyclique $\mathrm{E}_{\mathrm{r}}$ (de rechargement) au module $\mathrm{E}_{\mathrm{M}}$, soit la mesure directe du module cyclique. Les résultats sont identiques pour des dimensions transversales de semelles modestes. De grandes dimensions nécessitent de majorer ce module cyclique.

Si l'on n'a pour ainsi dire pas évoqué l'utilisation des modules issus des essais triaxiaux ou dynamiques, c'est que l'usage courant ne les consacre guère. Ces essais n'en restent pas moins des essais essentiels, et en particulier la " construction ») et la mise au point des lois de comportement, telle celles donnant $\frac{G}{G \max }$ en fonction de la distorsion $\gamma$ les rendent indispensables.

Mais le simple calcul de tassement des fondations peut s'effectuer à partir de données issues d'essais triaxiaux réalisés dans des conditions qui soient trans- 
posables pour les ouvrages. Cette pratique est courante dans de nombreux pays étrangers.

Pour l'estimation du tassement des sols compressibles sous charge uniforme de grande étendue, il est rappelé que la méthode œdométrique est la seule vraiment fiable qu'il y a lieu de privilégier. En revanche, l'application des expressions simples utilisant le module $\mathrm{E}_{\mathrm{M}}$ et le coefficient rhéologique $\alpha$ est tout à fait inadaptée pour ces sols et conduit à des valeurs dangereusement faibles, comme certains exemples réels le montrent. Les corrections qu'on peut être tenté d'appliquer sont entachées de grosses incertitudes. Au contraire, pour les sols « non compressibles , sable, grave, limons hors nappe, argile raide, l'estimation par cette même approche surestime fortement les tassements, même si en valeur absolue, ils peuvent s'avérer modérés. On propose donc pour ces sols de modifier la valeur $\alpha$, à diviser les tassements estimés actuellement par des coefficients variant de 2 à 5 .

On conçoit donc, qu'entre ces deux situations extrêmes il existe des sols où l'application du calcul pressiométrique conduit à des résultats corrects.

Quant aux soutènements souples (rideaux de palplanches, parois moulées), si, comme pour les semelles, la dimension influe sur le choix des modules, la valeur de ces derniers n'est pas non plus indépendante des performances attendues en termes de déformation horizontale. Plus le rapport de la flèche demandée à la hauteur soutenue est faible, plus le module le justifiant est fort et se rapproche du module élastique initial ; l'inertie seule du soutènement ne permet pas en effet d'assurer cette flèche,

On retiendra, dans la théorie pressiométrique, l'intérêt et la pertinence du coefficient $\alpha$, dans la prévi- sion de calcul des tassements des fondations superficielles traditionnelles.

Dès que la consolidation entre pour une part essentielle des tassements, ce coefficient est inapte à en rendre compte. Seul l'essai cedométrique en permet une approche correcte.

Quant à l'élasticité simple, elle peut s'avérer largement suffisante, le choix d'un module (ou de modules) étant cependant délicat. En particulier, déduire un module d'élasticité à partir de la valeur $\mathrm{E}_{\mathrm{M}}$ du module pressiométrique par la simple formule $E=\frac{E M}{\alpha}$, s'avère fausse et pessimiste. Une relation de type $E=k \frac{E M}{\alpha}$ est plus adaptée, où $k$ varie avec le sol, la dimension des ouvrages, et le niveau de déformation atteint, si ce niveau s'avère très faible. Le coefficient $\alpha$ perd donc de son intérêt dans cette approche. On pourra s'inspirer des tableaux VII donnés dans cette synthèse pour estimer $\mathrm{E}$.

Le module d'un sol n'est en réalité qu'un terme très générique, car il y a essentiellement des modules, variant avec les déformations, et de natures différentes suivant que le sol est cisaillé ou comprimé. Ces deux mécanismes se côtolent en permanence dans les massifs sous les sollicitations apportées par les ouvrages.

\footnotetext{
REMERCIEMENTS

Je tiens à remercier MM. M. Baudry, F. Schlosser, P. Schmitt, P. Vezole, F. Baguelin, M. Favre, B. Soyez, qui ont bien voulu me faire part de leurs avis et conseils. Un remerciement particulier śadresse á $M^{\text {me }}$ N. Daon du laboratoire régional des Ponts ef Chaussées de Rouen, laquelle a accepté d'assurer, sur près d'un an et demi, les frappes successives de cet article.
}

\section{Bibliographie}

AFNOR (NF P94117-1) - Portance des plates-formes, partie 1 : module sous chargement statique ả la plaque, EV2 avril 2000.

AFNOR (NF P94110-1) - Essai pressiométrique Ménard, partie 1: essai sans cycle, jarivier 2000

AFNOR (projet de norme francaise NF XP94110-2). - Essai pressiométrique Ménard, partie 2: essai avec cycle, 1999

Baquelin F., et al. - The pressumeter and foundation engineering. Trans Tech publications, 1978.

Balay J. - Recommandations pour le choix des paramétres de calcul des écrans de soutènement par la méthode aux modules de réaction. Note d'information technique LCPC, 1985.

Bretonnière S. - Étude d'un déflectomètre à boulet. Bulletin de liaison des laboratoires routiers des $P$ et $C n^{\circ} 2$, juillet-aoùt 1963, p. 43,5-43.16

Chassaing P., et al. - La dynaplaque 2 Etude d'un nouveau matériel. Bulletin de liajson des LPC $n^{\circ} 195$, janvier-février 1995, p. 3-13.

Combarieu 0. - Calcul d'une fondation mixte semelle-pieux sous charge verticale centrée. Note d'information technique, LCPC, 1988, $16 \mathrm{p}$.

Combarieu O., Canepa Y. - L'essai cyclique au pressiometre. Bulletin des $L P C \mathrm{n}^{\circ} 233$ p. 37 à 65 ; juillet-août 2001, p. 37-65.

Droniuc N., Magnan J.P, - Portance et tassements des fondations superficielles présentation des résultats dés concours de prévisions. FOND SUP 2003, vol. 2 Presses des Ponts, 2004, p. 401-416.

Essai pressiométrique normal - Modes opératoires du LCPC, Dunod, 1971

Essais de plaques et mécanique des chaus. sées. - Bulletin de liaison des laboratoires routiers LCPC, spécial B, février 1965

Frank R. - Quelques développements récents sur le comportement des fondations superficielles. $X^{e}$ Congrès européen de mécanique des sols et travaux de fondations, Florence, vol. 3 ; mai 1991, p. 1003-1030.

Garnier J. - Tassement et contraintes. Influence de la rigidité de la fondation et de l'anisotropie du massif. Thèse de doctorat, université de Grenoble, 15 juin 1973

Giroud J.-P. - Tables pour le calcul des fondations, Tomes 1 et 2. Dunod, 1973.

Giroud J.-P. - Tassement et stabilité des fondations superficielles. Tome 1 Presses universitaires de Grenoble. 1975.

Lavisse J., Schmitt P. - Interprétation des mesures de tassement sous des remblais de préchargement au Port de Rouen. Symposium International ASEP GI 2004: Paris, sept. 2004, p. 437 à 445.

Magnan J.-P., Bescond B., Serratrice J,-F, Déformabilité d'une marne chargée par des remblais. 2nd International Symposium on hard soils-soft rocks, Naples, 12 14 octobre 1998, p. 659-667.

Ménard L., Rousseau J. - L'évaluation des tassements, tendances nouvelles. Sols Soils n $1,2^{e}$ trim. 1962, p. 13-29.

Mestat Ph., Reiffsteck Ph. - Modules de déformation en mécanique des sols définitions, détermination à partir des essais triaxiaux et incertitude. PARAM 2002, septembre 2002, p. 392-400.

Morel G.. Quibel A. - Le portancemètre un nouvel appareil d'auscultation en continu des couches de forme et de la partie superieure des terrassements. Revue générale des routes et aérodromes, $n^{\circ} 768$, décembre 1998 , p. 106 109

Nguyen Phuong D., et al. - Deux modélisations par éléments finis d'écrans de soutenement instrumentés. Bulletin des LPC, jarnv-fév.-mars 2005, p. 41-59.

Pham Van Bang D. - Comportement instantané et différé des sables, des petites aux moyennes déformations: expérimentation et modélisation. Thèse soutenue à I'INSA de Lyon, 28 avril 2004.

Pouya A., Reiffsteck Ph. - Solutions fondamentales pour fondations sur sols élas tiques anisotropes. FONDSUP 2003, vol. 1, 2003, p. 415-422.

Serrai K., et al. - Interprétation par la méthode des éléments finis des mesures de déformation d'une paroi moulée à Colombes. Congrès international des fondations et de mécanique des sols, Osaka, vol. 2, septembre 2005, p. 1119-1123.

Utilisation du vibreur Goodman. - Bulletin de liaison des LPC, spécial J, juillet 1968. 